\title{
Shock Diffraction by Convex Cornered Wedges for the Nonlinear Wave System
}

\author{
Gui-Qiang G. Chen Xuemei Deng Wei Xiang \\ Accepted for publication in [Arch. Rational Mech. Anal.]
}

November 27, 2018

\section{INTRODUCTION}

We are concerned with rigorous mathematical analysis of shock diffraction by two-dimensional cornered wedges whose angles are less than $\pi$ in compressible fluid flow, through the nonlinear wave system. The study of the shock diffraction problem dates back to the 1950's and starts from the work of Bargman [2], Lighthill [18, 19], Fletcher-Weimer-Bleakney [10], and Fletcher-Taub-Bleakney [9] via asymptotic or experimental analysis. Also see Courant-Friedrichs [8] and Whitham [21.

In this paper, we develop several mathematical ideas and techniques through the nonlinear wave system to establish a rigorous theory of existence and regularity of solutions to the diffraction problem. The nonlinear wave system consists of three conservation laws, which takes the form:

$$
\begin{aligned}
\rho_{t}+m_{x_{1}}+n_{x_{2}} & =0, \\
m_{t}+p_{x_{1}} & =0, \\
n_{t}+p_{x_{2}} & =0,
\end{aligned}
$$

for $(t, \mathbf{x}) \in[0, \infty) \times \mathbb{R}^{2}, \mathbf{x} \in \mathbb{R}^{2}$, where $\rho$ stands for the density, $p$ for the pressure, $(m, n)$ for the momenta in the $\left(x_{1}, x_{2}\right)$-coordinates. The pressure-density constitutive relation is

$$
p(\rho)=\rho^{\gamma} / \gamma, \quad \gamma>1,
$$

by scaling without loss of generality. Then the sonic speed $c=c(\rho)$ is determined by

$$
c^{2}(\rho):=p^{\prime}(\rho)=\rho^{\gamma-1} .
$$

Notice that $c(\rho)$ is a positive, increasing function for all $\rho>0$.

The two-dimensional nonlinear wave system (1.1) is derived from the compressible isentropic gas dynamics by neglecting the inertial terms, i.e., the quadratic terms in the velocity; see Canic-Keyfitz-Kim [4]. Also see Zheng [22] for a related hyperbolic system, the pressure gradient system of conservation laws; the same arguments developed in this paper can be carried through to establish a corresponding theory of existence and regularity for the pressure gradient system. 
Let $S_{0}$ be the vertical planar shock in the $(t, \mathbf{x})$-coordinates, $t \in \mathbb{R}_{+}:=[0, \infty), \mathbf{x}=$ $\left(x_{1}, x_{2}\right) \in \mathbb{R}^{2}$, with the left constant state $U_{1}=\left(\rho_{1}, m_{1}, 0\right)$ and the right state $U_{0}=\left(\rho_{0}, 0,0\right)$, satisfying

$$
m_{1}=\sqrt{\left(p\left(\rho_{1}\right)-p\left(\rho_{0}\right)\right)\left(\rho_{1}-\rho_{0}\right)}>0, \quad \rho_{1}>\rho_{0} .
$$

When $S_{0}$ passes through a convex cornered wedge:

$$
W:=\left\{\left(x_{1}, x_{2}\right): x_{2}<0,-\infty<x_{1} \leq x_{2} \operatorname{ctan} \theta_{w}\right\},
$$

shock diffraction occurs, where the wedge angle $\theta_{w}$ is between $-\pi$ and 0 ; see Fig. 1 . Then the shock diffraction problem can be formulated as the following mathematical problem:
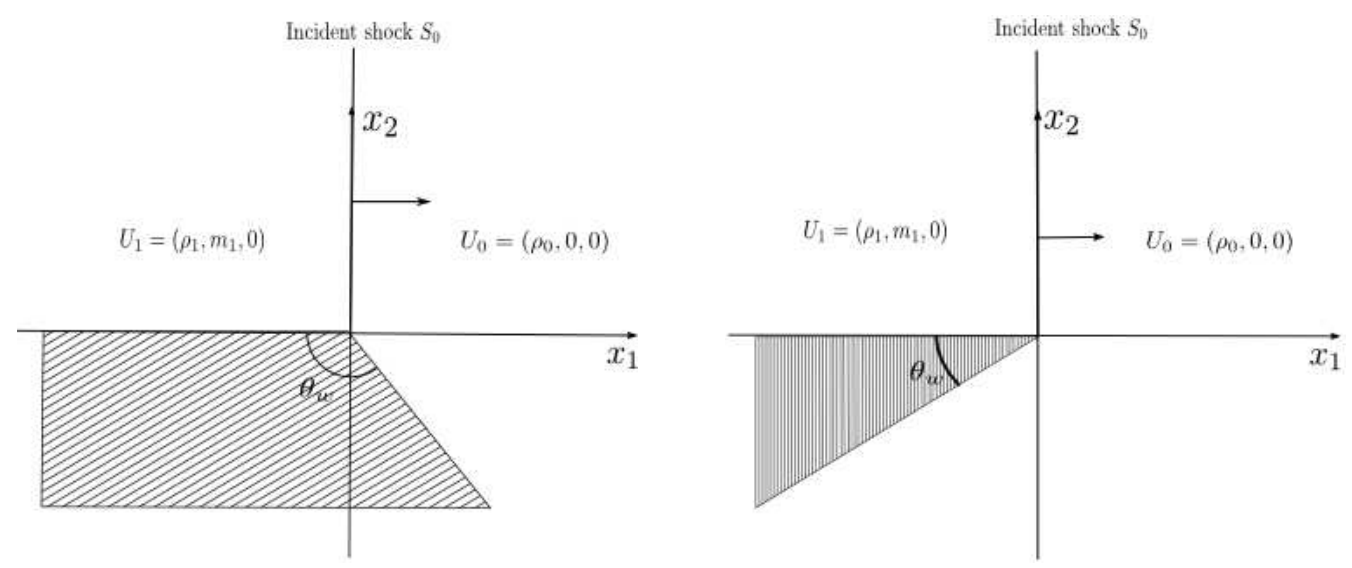

Figure 1. Initial-boundary value problem

Problem 1 (Initial-Boundary Value Problem). Seek a solution of system (1.1) with the initial condition at $t=0$ :

$$
\left.(\rho, m, n)\right|_{t=0}= \begin{cases}\left(\rho_{0}, 0,0\right) & \text { in }\left\{-\pi+\theta_{w} \leq \arctan \left(\frac{x_{2}}{x_{1}}\right) \leq \frac{\pi}{2}\right\}, \\ \left(\rho_{1}, m_{1}, 0\right) & \text { in }\left\{x_{1}<0, x_{2}>0\right\},\end{cases}
$$

and the slip boundary condition along the wedge boundary $\partial W$ :

$$
\left.(m, n) \cdot \boldsymbol{\nu}\right|_{\partial W}=0,
$$

where $\boldsymbol{\nu}$ is the exterior unit normal to $\partial W$ (see Fig. 1).

Notice that the initial-boundary value problem (1.1)-(1.4) is invariant under the self-similar scaling:

$$
(t, \mathbf{x}) \rightarrow(\alpha t, \alpha \mathbf{x}) \quad \text { for } \quad \alpha \neq 0 .
$$

Thus, we seek self-similar solutions with the form

$$
(\rho, m, n)(t, \mathbf{x})=(\rho, m, n)(\xi, \eta) \quad \text { for } \quad(\xi, \eta)=\frac{\mathbf{x}}{t} .
$$


In the self-similar coordinates $(\xi, \eta)$, system (1.1) can be rewritten as

$$
\begin{aligned}
(m-\xi \rho)_{\xi}+(n-\eta \rho)_{\eta}+2 \rho & =0, \\
(p(\rho)-\xi m)_{\xi}-(\eta m)_{\eta}+2 m & =0, \\
(\xi n)_{\xi}-(p(\rho)-\eta n)_{\eta}-2 n & =0 .
\end{aligned}
$$

In the polar coordinates $(r, \theta), r=\sqrt{\xi^{2}+\eta^{2}}$, the system can be further written as (1.8)

$$
\partial_{r}\left(\begin{array}{c}
r \rho-\cos \theta m-\sin \theta n \\
r m-\cos \theta p(\rho) \\
r n-\sin \theta p(\rho)
\end{array}\right)+\partial_{\theta}\left(\begin{array}{c}
\sin \theta m-\cos \theta n \\
\sin \theta p(\rho) \\
-\cos \theta p(\rho)
\end{array}\right)=\left(\begin{array}{c}
\rho+\frac{\cos \theta}{r} m+\frac{\sin \theta}{r} n \\
m+\frac{\cos \theta}{r} p(\rho) \\
n+\frac{\sin \theta}{r} p(\rho)
\end{array}\right) .
$$

The location of the incident shock $S_{0}$ for large $r \gg 1$ is:

$$
\xi=\xi_{1}=\sqrt{\frac{p\left(\rho_{1}\right)-p\left(\rho_{0}\right)}{\rho_{1}-\rho_{0}}}>0 .
$$

Then Problem 1 can be reformulated as a boundary value problem in an unbounded domain:

Problem 2 (Boundary Value Problem). Seek a solution of system (1.7), or equivalently (1.8), with the asymptotic boundary condition when $r \rightarrow \infty$ :

$$
(\rho, m, n) \rightarrow \begin{cases}\left(\rho_{0}, 0,0\right) & \text { in }\left\{\xi>\xi_{1}, \eta>0\right\} \cup\left\{-\pi+\theta_{w} \leq \arctan \left(\frac{\eta}{\xi}\right) \leq 0\right\}, \\ \left(\rho_{1}, m_{1}, 0\right) & \text { in }\left\{\xi<\xi_{1}, \eta>0\right\},\end{cases}
$$

and the slip boundary condition along the wedge boundary $\partial W$ :

$$
\left.(m, n) \cdot \boldsymbol{\nu}\right|_{\partial W}=0,
$$

where $\boldsymbol{\nu}$ is the exterior unit normal to $\partial W$ (see Fig. 2).
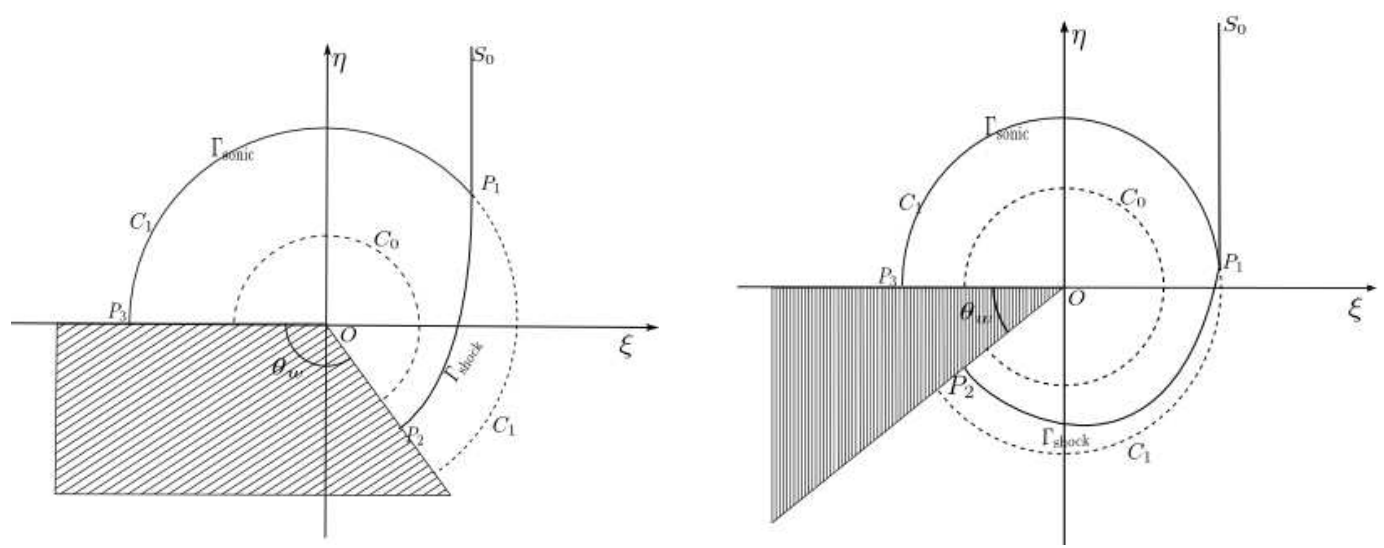

FiguRE 2. Shock diffraction configuration 
For a smooth solution $U=(\rho, m, n)$ to (1.7), we may eliminate $m$ and $n$ in (1.1) to obtain a second-order nonlinear equation for $\rho$ :

$$
\left(\left(c^{2}-\xi^{2}\right) \rho_{\xi}-\xi \eta \rho_{\eta}+\xi \rho\right)_{\xi}+\left(\left(c^{2}-\eta^{2}\right) \rho_{\eta}-\xi \eta \rho_{\xi}+\eta \rho\right)_{\eta}-2 \rho=0 .
$$

Correspondingly, equation (1.12) in the polar coordinates $(r, \theta), r=\sqrt{\xi^{2}+\eta^{2}}$, takes the form

$$
\left(\left(c^{2}-r^{2}\right) \rho_{r}\right)_{r}+\frac{c^{2}}{r} \rho_{r}+\left(\frac{c^{2}}{r^{2}} \rho_{\theta}\right)_{\theta}=0 .
$$

In the self-similar coordinates, as the incident shock $S_{0}$ passes through the wedge corner, $S_{0}$ interacts with the sonic circle $\Gamma_{\text {sonic }}$ of state $(1): r=r_{1}$, and becomes a transonic diffracted shock $\Gamma_{\text {shock}}$, and the flow in the domain $\Omega$ behind the shock and inside $\Gamma_{\text {sonic }}$ becomes subsonic. In Section 2, we reduce Problem 2 for shock diffraction into a one-phase free boundary problem, Problem 3, for second-order elliptic equation in the domain $\Omega$ with the free boundary $\Gamma_{\text {shock}}$, degenerate boundary $\Gamma_{\text {sonic }}$, and slip boundary $\partial W \cap \Omega$. In this paper, we focus on the existence of global solutions of shock diffraction and the optimal regularity of the solution across the sonic circle $\Gamma_{\text {sonic }}$.

There are two additional difficulties to establish the global existence of solutions, besides the ellipticity degenerates at the sonic circle $\Gamma_{\text {sonic }}$. The first is that the oblique derivative boundary condition degenerates at $P_{2}$, that is, $\beta_{2}$ may equal to 0 , for which a one-point Dirichlet boundary condition has to be identified to ensure the uniqueness of solutions. The second difficulty is that the diffracted shock may coincide with the sonic circle $C_{0}:=\left\{r=c\left(\rho_{0}\right)\right\}$ of state (0) in the iteration where the oblique derivative boundary condition fails again. Then we can not employ directly the results in Liebermann [14]-17] to show the existence of solutions for the fixed boundary value problem. One of our strategies here is to enforce an additional condition $r(\theta) \geq c\left(\rho_{0}\right)+\delta$ on the diffracted shock curve with $\delta$ small enough and modify slightly the approximate shock curve to overcome the difficulty.

The approach used in this paper for establishing the global existence of solutions is first to regularize the equation by adding the regularized differential operator $\varepsilon \Delta(\Delta$ denotes the Laplace operator in the self-similar coordinates) to make the equation uniformly elliptic; and then to rely on the Perron method, as in [13], to show the global existence of solutions for the fixed boundary value problem; and finally to apply the Schauder fixed point theorem to show the existence of global solutions for the free boundary problem. Moreover, we obtain uniform estimates for the global solutions with respect to $\delta, \varepsilon>0$ so that we can pass the limits $\delta \rightarrow 0$ and $\varepsilon \rightarrow 0$ to establish the existence of solutions of the free boundary problem for the original system. In particular, we prove that the diffracted shock is uniformly transonic, that is, the strength of the shock is positive even at point $P_{2}$.

In order to establish the optimal regularity across the sonic boundary $\Gamma_{\text {sonic }}$, we write equation (1.13) in terms of the function

$$
\psi:=c^{2}\left(\rho_{1}\right)-c^{2}(\rho)
$$


in the $(x, y)$-coordinates, which is specified in $\S 5$, defined near $\Gamma_{\text {sonic }}$ such that $\Gamma_{\text {sonic }}$ becomes a segment on $\{x=0\}$, with the form

$$
\left(2 c_{1} x-\psi\right) \psi_{x x}+\psi_{y y}+c_{1} \psi_{x}-\psi_{x}^{2}-\frac{1}{(\gamma-1) c_{1}^{2}} \psi_{y}^{2}=0 \quad \text { in } x>0 \text { and near } x=0,
$$

plus "small" terms, since $\rho$ and $\psi$ have the same regularity in $\Omega$. Then we employ the approach in Bae-Chen-Feldman [1] to analyze the features of equation (1.14) and prove the $C^{1, \alpha}$-regularity of solutions of the shock diffraction problem in the elliptic region up to part $\overline{\Gamma_{\text {sonic }}} \backslash P_{1}$ of the sonic shock. As a corollary, we establish that the $C^{0,1}$-regularity is actually optimal across the sonic boundary $\Gamma_{\text {sonic }}$ from the elliptic region $\Omega$ to the hyperbolic region of state (1), that is, the optimal regularity at the degenerate elliptic boundary.

We remark that the existence problem for a shock interaction with the right cornered wedge (90-degree) was studied by Kim [12, in which some important features and behavior of solutions have been exhibited. As far as we have known, for the shock diffraction by a convex cornered wedges whose angles are between $-\pi$ and 0 in compressible fluid flow, no rigorous complete global mathematical results have been available, since the early work by Bargman [2, Lighthill [18, 19], FletcherTaub-Bleakney [9], and Fletcher-Weimer-Bleakney [10]. The results established in this paper is the first rigorous complete mathematical results through the nonlinear wave system for the global existence and optimal regularity of solutions of shock diffraction by any convex cornered wedge.

A closely related problem, shock reflection-diffraction by a concave cornered wedges, has been systematically analyzed in Chen-Feldman [5, 6, 7] and Bae-Chen-Feldman [1], where the existence of regular shock reflection-diffraction configurations has been established up to the sonic wedge-angle for potential flow. Also see Canic-KeyfitzKim [3, 4] for the unsteady transonic small disturbance equation and the nonlinear wave system, Zheng [22] for the pressure-gradient system, and Serre [20] for the Euler equations for a Chaplygin gas.

The organization of this paper is as follows. In $\S 2$, we reformulate the shock diffraction problem into a free boundary problem for the nonlinear second-order equation (1.1) in both the self-similar and polar coordinates, and present the statement of our main theorem for the existence and optimal regularity of the global solution. In $\S 3$, we first formulate the regularized approximate free boundary problem by adding a regularized differential operator with $\varepsilon \Delta \rho$ to the original equation and the assumption $c(\bar{\rho}) \geq c\left(\rho_{0}\right)+\delta$, where $\bar{\rho}$ is the data given at point $P_{2}$. Then we establish the existence of solutions to the regularized free boundary problem for the uniformly elliptic equation in the polar coordinates, and so does in the self-similar coordinates, as approximate solutions to the original free boundary problem. In $\S 4$, we proceed to the limits $\varepsilon \rightarrow 0$ and $\delta \rightarrow 0$ to establish the global existence of solutions of the original problem in the self-similar coordinates. In $\S 5$, we establish the optimal $C^{0,1}$-regularity of the solution $\rho$ across the degenerate sonic boundary. In $\S 6$, we establish a corresponding theorem for the existence and regularity of solutions of the shock diffraction problem for the nonlinear wave system. 


\section{Mathematical Formulation and Main Theorem}

In this section, we derive mathematical formulation of the shock diffraction problem as a free boundary problem for a nonlinear degenerate elliptic equation of second order and present our main theorem of this paper. In particular, we employ the Rankine-Hugoniot relations to set up a boundary condition along the free boundary (shock) and derive other boundary conditions along the wedge boundaries in the polar coordinates.

2.1. Rankine-Hugoniot Conditions and Oblique Derivative Boundary Condition on the Diffracted Shock. Consider system (1.8) in the polar coordinates. Then the Rankine-Hugoniot relations, i.e., the jump conditions, are

$$
\begin{aligned}
& \frac{d r}{d \theta}=r \frac{\sqrt{r^{2}-\bar{c}^{2}\left(\rho, \rho_{0}\right)}}{\bar{c}\left(\rho, \rho_{0}\right)}, \\
& {[p][\rho]=[m]^{2}+[n]^{2},}
\end{aligned}
$$

with $\bar{c}\left(\rho, \rho_{0}\right)=\sqrt{\frac{p(\rho)-p\left(\rho_{0}\right)}{\rho-\rho_{0}}}$, where we have chosen the plus branch so that $\frac{d r}{d \theta}>0$. Differentiating (2.2) along $\Gamma_{\text {shock }}$ and using the equations obtained above with careful calculation, we finally obtain

$$
\sum_{i=1}^{2} \beta_{i} D_{i} \rho:=\beta_{1} \rho_{r}+\beta_{2} \rho_{\theta}=0,
$$

where $\beta=\left(\beta_{1}, \beta_{2}\right)$ is a function of $\left(\rho_{0}, \rho, r(\theta), r^{\prime}(\theta)\right)$ with

$$
\beta_{1}=r^{\prime}(\theta)\left(c^{2}\left(r^{2}-\bar{c}^{2}\right)-3 \bar{c}^{2}\left(c^{2}-r^{2}\right)\right), \quad \beta_{2}=3 c^{2}\left(r^{2}-\bar{c}^{2}\right)-\bar{c}^{2}\left(c^{2}-r^{2}\right) .
$$

Thus, the obliqueness becomes

$$
0 \neq \beta \cdot\left(1,-r^{\prime}(\theta)\right)=-2 r^{2}\left(c^{2}-\bar{c}^{2}\right) r^{\prime}(\theta)=: \mu,
$$

where $\left(1,-r^{\prime}(\theta)\right)$ is the outward normal to $\Omega$ on $\Gamma_{\text {shock }}$. Note that $\mu$ becomes zero when $r^{\prime}(\theta)=0$, that is, $r=\bar{c}\left(\rho, \rho_{0}\right)$. When the obliqueness fails, we have

$$
\beta_{1}=0, \quad \beta_{2}=-\bar{c}^{2}\left(c^{2}-r^{2}\right)<0,
$$

since $c^{2}(\rho)>\bar{c}^{2}\left(\rho, \rho_{0}\right)=r^{2}$ if $\rho>\rho_{0}$.

We define $Q$ to be the governing second-order quasilinear operator in the subsonic domain $\Omega$ :

$$
Q \rho:=\left(\left(c^{2}-r^{2}\right) \rho_{r}\right)_{r}+\frac{c^{2}}{r} \rho_{r}+\left(\frac{c^{2}}{r^{2}} \rho_{\theta}\right)_{\theta}=0,
$$

and $M$ to be the oblique derivative boundary operator:

$$
M \rho:=\beta_{1} \rho_{r}+\beta_{2} \rho_{\theta}=0 \quad \text { on } \quad \Gamma_{\text {shock }}:=\left\{(r(\theta), \theta): \theta_{w} \leq \theta \leq \theta_{1}\right\} .
$$

The second condition on $\Gamma_{\text {shock }}$ is the shock evolution equation:

$$
\frac{d r}{d \theta}=r \frac{\sqrt{r^{2}-\bar{c}^{2}\left(\rho, \rho_{0}\right)}}{\bar{c}\left(\rho, \rho_{0}\right)}:=g(r, \theta, \rho(r, \theta)), \quad r\left(\theta_{1}\right)=r_{1},
$$


where $\left(r_{1}, \theta_{1}\right)$ are the polar coordinates of $P_{1}=\left(\xi_{1}, \eta_{1}\right)$.

At point $P_{2}, r^{\prime}\left(\theta_{w}\right)=0, M$ does not satisfy the oblique derivative boundary condition at this point. We may alternatively express this as a one-point Dirichlet condition by solving $r\left(\theta_{w}\right)=\bar{c}\left(\rho\left(r\left(\theta_{w}\right), \theta_{w}\right), \rho_{0}\right)$. In order to deal with this equation, we introduce the notation:

$$
a=\left(\bar{c}_{b}\right)^{-1}(r) \quad \text { when } \bar{c}_{b}:=\bar{c}(a, b)=r \text { for fixed } b .
$$

Thus, we have

$$
\rho\left(P_{2}\right)=\bar{\rho}=\left(\bar{c}_{\rho_{0}}\right)^{-1}\left(r\left(\theta_{w}\right)\right) .
$$

2.2. Boundary Condition on the Wedge. The boundary condition on the wedge is the slip boundary condition, i.e., $(m, n) \cdot \boldsymbol{\nu}=0$. Differentiating it along the wedge, and combining this with the second and third equations in (1.1), we conclude that $\rho$ satisfies

$$
\rho_{\nu}=0 \quad \text { on } \Gamma_{0}:=\partial \Omega \cap\left(\{\theta=\pi\} \cup\left\{\theta=\theta_{w}\right\}\right) .
$$

2.3. Boundary Condition on $\Gamma_{\text {sonic }}$ of State (1). The Dirichelt boundary condition on $\Gamma_{\text {sonic }}$ :

$$
\rho=\rho_{1} \quad \text { on } \Gamma_{\text {sonic }}:=\partial \Omega \cap \partial B_{c_{1}}(0) .
$$

On the Dirichlet boundary $\Gamma_{\text {sonic }}$, the equation $Q \rho=0$ becomes degenerate elliptic from the inside of $\Omega$.

2.4. Reformulation of the Shock Diffraction Problem. With the derivation of the free boundary condition on $\Gamma_{\text {shock }}$ and the fixed boundary conditions on $\Gamma_{\text {sonic }}$ and the wedge $\Gamma_{0}$, Problem 2 is reduced to the following free boundary problem in the domain $\Omega$ for the second order equation (2.6), with $(m, n)$ correspondingly determined by (1.8).

Problem 3 (Free Boundary Problem). Seek a solution of the second-order nonlinear equation (2.6) for the density function $\rho$ in the domain $\Omega$, satisfying the free boundary conditions (2.7)-(2.10) on $\Gamma_{\text {shock}}$, the Neumann boundary condition (2.11) on the wedge $\Gamma_{0}$, and the Dirichlet boundary condition (2.12) on the degenerate boundary $\Gamma_{\text {sonic }}$, the sonic circle of state (1) (cf. Fig. 2).

2.5. Main Theorem. For the free boundary problem, Problem 3, we have the following results, which form the main theorem of this paper.

Theorem 2.1 (Main Theorem). Let the wedge angle $\theta_{w}$ be between $-\pi$ and 0 . Then there exists a global solution $\rho(r, \theta)$ in the domain $\Omega$ with the free boundary $r=r(\theta), \theta \in\left[\theta_{w}, \theta_{1}\right]$, of Problem 3:

$$
\rho \in C^{2+\alpha}(\Omega) \cap C^{\alpha}(\bar{\Omega}), \quad r \in C^{2+\alpha}\left(\left[\theta_{w}, \theta_{1}\right)\right) \cap C^{1,1}\left(\left[\theta_{w}, \theta_{1}\right]\right) .
$$

Moreover, the solution $(\rho(r, \theta), r(\theta))$ satisfies the following properties:

(i) $\rho>\rho_{0}$ on the shock $\Gamma_{\text {shock}}$, that is, the shock $\Gamma_{\text {shock }}$ is separated from the sonic circle $C_{0}$ of state (0); 
(ii) The shock $\Gamma_{\text {shock }}$ is strictly convex up to point $P_{1}$, except point $P_{2}$, in the self-similar coordinates $(\xi, \eta)$;

(iii) The solution is $C^{1, \alpha}$ up to $\Gamma_{\text {sonic }}$ and Lipschitz continuous across $\Gamma_{\text {sonic }}$;

(iv) The Lipschitz regularity of solutions across $\Gamma_{\text {sonic }}$ and at $P_{1}$ from the inside is optimal.

In particular, Theorem 2.1 implies the following facts:

(a) The diffracted shock $\Gamma_{\text {shock }}$ definitely is not degenerate at point $P_{2}$. This has been an open question even when the wedge angle is $\frac{\pi}{2}$ as in [12, though it is physically plausible.

(b) The curvature of the diffracted shock $\Gamma_{\text {shock }}$ away from point $P_{2}$ is strictly convex and has a jump at point $P_{1}$ from a positive value to zero, while the strict convexity of the curvature fails at $P_{2}$.

(c) The optimal regularity of solutions across $\Gamma_{\text {sonic }}$ and at $P_{1}$ from the inside is $C^{0,1}$, i.e., Lipschitz continuity.

We establish Theorem 2.1 in two main steps. First, we solve the regularized approximate free boundary problem for $Q$ involving two small parameters $\varepsilon$ and $\delta$, introduced in $\S 3$. Then we analyze the limits $\varepsilon \rightarrow 0$ and $\delta \rightarrow 0$, and prove that the limits yield a solution of Problem 3, i.e., (2.6)-(2.12), in $\S 4$. The optimal regularity is established in $\S 5$.

\section{Regularized Approximate Problem}

In this section we first formulate the regularized approximate free boundary problem and establish the existence of solutions to this problem as approximate solutions to the original problem. To solve the free boundary problem, we formulate the fixed point argument in terms of the position of the free boundary. There are two main difficulties in establishing the existence of solutions: The first is that the ellipticity degenerates at the sonic circle $\Gamma_{\text {sonic }}$; and the second is that the free boundary $\Gamma_{\text {shock }}$ may coincide with the sonic circle $C_{0}$ of state (0) in an iteration, which would make the iteration impossible. We overcome these difficulties as described below.

3.1. Approximate Problem and Existence Theorem for Approximate Solutions. For fixed $\varepsilon>0$, introduce a regularized operator:

$$
Q^{\varepsilon}:=Q+\varepsilon \triangle
$$

where $\triangle$ represents the Laplace operator in the self-similar coordinates. For a given curve $r(\theta)$, we first solve the fixed boundary value problem (2.6) -(2.7), (2.11)-(2.12), and $(2.10)$ with $Q$ replaced by $Q^{\varepsilon}$; then we obtain a new shock position $\tilde{r}(\theta)$ by integrating (2.8):

$$
\tilde{r}(\theta)=r_{1}+\int_{\theta_{1}}^{\theta} g(r(s), s, \rho(s, r(s))) d s \quad \text { for } \theta \in\left[\theta_{w}, \theta_{1}\right),
$$

where $g$ is defined in (2.8). Note that, on the right side of (3.1), we evaluate all the quantities along the old shock position $r(\theta)$. 
With this, it seems that the free boundary could be obtained by solving a fixed boundary problem and then by integrating the shock evolution equation. However, we face the second difficulty as indicated above, that is, $\tilde{r}(\theta)$ may meet the sonic circle $C_{0}$ of state $(0)$. To overcome the new difficulty, we introduce another small, positive parameter $\delta$ which is fixed and define the iteration set of $r, \mathcal{K}^{\varepsilon, \delta}$, which is a closed, convex subset of the Hölder space $C^{1+\alpha_{1}}\left(\left[\theta_{w}, \theta_{1}\right]\right)$, where $\alpha_{1}$ depends on $\varepsilon$ and $\delta$ to be specified later. The functions in $\mathcal{K}^{\varepsilon, \delta}$ satisfy

$\left(K_{1}\right) \quad r\left(\theta_{1}\right)=r_{1}$

$\left(K_{2}\right) \quad r^{\prime}\left(\theta_{w}\right)=0$

$\left(K_{3}\right) \quad c\left(\rho_{0}\right)+\delta \leq r\left(\theta_{w}\right)$;

$\left(K_{4}\right) \quad 0 \leq r^{\prime}(\theta) \leq \frac{r_{1}^{2}}{\bar{c}\left(\rho_{0}\right)}$ for $\theta_{w} \leq \theta \leq \theta_{1}$.

When the difficulty occurs, we modify $\tilde{r}(\theta)$ slightly somewhere as $r(\theta)=c\left(\rho_{0}\right)+\delta+$ $A\left(\theta-\theta_{w}\right)^{3}+B\left(\theta-\theta_{w}\right)^{n}$, where $A, B$, and $n$ will be uniquely determined. Then we define a mapping on $\mathcal{K}^{\varepsilon, \delta}$ :

$$
J: r \rightarrow \tilde{r}
$$

We now restate the regularized approximate problem as follows: For fixed $\varepsilon, \delta>0$, the equation for $\rho$ in the subsonic region is

$$
Q^{\varepsilon} \rho=\left(\left(c^{2}-r^{2}+\varepsilon\right) \rho_{r}\right)_{r}+\frac{c^{2}+\varepsilon}{r} \rho_{r}+\left(\frac{c^{2}+\varepsilon}{r^{2}} \rho_{\theta}\right)_{\theta}=0
$$

the shock evolution equation remains the same when $r \geq c\left(\rho_{0}\right)+2 \delta$ :

$$
\left\{\begin{array}{l}
\frac{d r}{d \theta}=g(r, \theta, \rho), \\
r\left(\theta_{1}\right)=r_{1}
\end{array}\right.
$$

and

$$
r(\theta)=c\left(\rho_{0}\right)+\delta+A\left(\theta-\theta_{w}\right)^{3}+B\left(\theta-\theta_{w}\right)^{n}
$$

for some constants $A, B$, and $n$ on the boundary when (3.3) does not hold; the remaining boundary conditions as before are

$$
\begin{gathered}
M \rho=\beta \cdot \nabla \rho=0 \quad \text { on } \Gamma_{\text {shock }}=\left\{(r, \theta): \theta_{w}<\theta<\theta_{1}\right\}, \\
\rho=\rho_{1} \quad \text { on } \Gamma_{\text {sonic }}, \quad \rho_{\nu}=0 \quad \text { on } \Gamma_{0},
\end{gathered}
$$

where $\boldsymbol{\nu}$ is the outward normal to $\Omega$ at $\Gamma_{0}$; and

$$
\rho\left(P_{2}\right)=\bar{\rho}=\left(\bar{c}_{\rho_{0}}\right)^{-1}\left(r\left(\theta_{w}\right)\right) .
$$

Let $V=\left\{P_{1}, P_{2}, O, P_{3}\right\}$ denote the corners of $\Omega$, and $V^{\prime}=V \backslash\left\{P_{2}\right\}$. Set $\Omega^{\prime}=$ $\bar{\Omega} \backslash\left(V \cup \Gamma_{\text {shock }}\right)$. For $P \in V$, we define the corner region

$$
\Omega_{P}(\sigma):=\{x \in \Omega: \operatorname{dist}(x, P) \leq \sigma\}, \quad \Omega_{V}(\sigma):=\cup_{P \in V} \Omega_{P}(\sigma) .
$$


We define a region that is close to $\Gamma_{\text {shock}}$, but does not contain corner $P_{1}$ by taking a covering of $\Gamma_{\text {shock }}$ with a ball of radius $\delta$ centered at the points on $\Gamma_{\text {shock }}$ which are bounded away from $P_{1}$. Define

$$
\Gamma^{\prime}(\sigma):=\left\{P \in \Gamma_{\text {shock }}: \operatorname{dist}\left(P, P_{1}\right)>\sigma\right\}
$$

and

$$
\Gamma(\sigma)=\left\{x \in \Omega \cap\left(\cup_{P \in \Gamma^{\prime}(\sigma)} B_{\sigma}(P)\right)\right\},
$$

where $B_{\sigma}(P)$ is a ball of radius $\sigma$ centered at $P$. We then define

$$
C_{b}^{a} \equiv\left\{u:\|u\|_{a}^{b}:=\sup _{\sigma>0}\left(\sigma^{a+b}\|u\|_{a, \bar{\Omega} \backslash\left(\Gamma(\sigma) \cup \Omega_{V^{\prime}}(\sigma)\right)}\right)<\infty\right\} .
$$

We focus now on the proof of the following existence theorem in this section.

Theorem 3.1. For any $\varepsilon \in\left(0, \varepsilon_{0}\right)$ and $\delta \in\left(0, \delta_{0}\right)$ for some $\varepsilon_{0}, \delta_{0}>0$, there exists a solution $\left(\rho^{\varepsilon, \delta}, r^{\varepsilon, \delta}\right) \in C_{\left(-\gamma_{1}\right)}^{2+\alpha}\left(\Omega^{\varepsilon, \delta}\right) \times C^{1+\alpha}\left(\left[\theta_{w}, \theta_{1}\right]\right)$ to the regularized free boundary problem (3.2) - (3.7) such that

$$
\rho_{0}<\bar{\rho}^{\varepsilon, \delta} \leq \rho^{\varepsilon, \delta}<\rho_{1}, \quad c^{2}\left(\rho^{\varepsilon, \delta}\right) \geq r^{2} \quad \text { in } \bar{\Omega}^{\varepsilon, \delta}
$$

for some $\alpha, \gamma \in(0,1)$, which depend on $\varepsilon, \delta$, and the data $\left(\rho_{0}, \rho_{1}, \theta_{w}\right)$. Furthermore, the solution satisfies (3.3) at the points of $\Gamma_{\text {shock }}^{\varepsilon, \delta}$ where $r^{\varepsilon, \delta} \geq c\left(\rho_{0}\right)+2 \delta$. The function $r^{\varepsilon, \delta}(\theta)$, defining the position of the free boundary $\Gamma_{\text {shock }}^{\varepsilon, \delta}$, is in $\mathcal{K}^{\varepsilon, \delta}$. Here $\Omega^{\varepsilon, \delta}$ is bounded by $\Gamma_{\text {shock }}^{\varepsilon, \delta}, \Gamma_{\text {sonic }}$, and $\Gamma_{0}$.

We establish Theorem 3.1 in the following steps whose details are given in the following four subsections.

Step 1. Since the governing equation (3.2) is nonlinear and the ellipticity is not known a priori, we impose a cut-off function in the equation $Q^{\varepsilon} \rho=0$.

We introduce a smooth increasing function $\zeta \in C^{\infty}$ such that

$$
\zeta(s):= \begin{cases}s & \text { if } s \geq 0, \\ -\frac{1}{2} \varepsilon & \text { if } s<-\varepsilon,\end{cases}
$$

and $\left|\zeta^{\prime}(s)\right| \leq 1$. We then consider the following modified governing equation:

$$
\begin{aligned}
Q^{\varepsilon,+} \rho & =\left(\left(\zeta\left(c^{2}-r^{2}\right)+\varepsilon\right) \rho_{r}\right)_{r}+\left(\frac{c^{2}+\varepsilon}{r^{2}} \rho_{\theta}\right)_{\theta}+\left(\frac{1}{r}\left(\zeta\left(c^{2}-r^{2}\right)+\varepsilon\right)+r\right) \rho_{r} \\
& =\sum_{i=1}^{2} D_{i}\left(a_{i i}^{\varepsilon}(r, \theta, \rho) D_{i} \rho\right)+b^{\varepsilon}(r, \rho) D_{r} \rho=0 \quad \text { in } \Omega .
\end{aligned}
$$

Step 2. We make some estimates for a solution to the linear problem with fixed boundary $\Gamma_{\text {shock }}$ defined by $r(\theta) \in \mathcal{K}^{\varepsilon, \delta}$ and establish the Schauder estimates on $\Gamma_{\text {shock }}$.

Step 3. We employ a technique in Lieberman 13 to solve the problem with the oblique derivative boundary condition $M \rho=0$. Using the Hölder gradient bounds to the linear problem, we establish the existence results for the linear fixed boundary problem in the polar coordinates, via the Perron method developed in [13]. 
Step 4. We apply the Schauder fixed point theorem to conclude the existence of a solution to the free boundary problem with the oblique derivative boundary condition. Finally we remove the cut-off function by the a priori estimates to conclude the results.

\subsection{Proof of Theorem [3.1; Regularized Linear Fixed Boundary Value}

Problem. Replace $\rho$ in the coefficients $a_{i i}^{\varepsilon}$ and $b^{\varepsilon}$ in (3.11) and $\beta_{i}$ in (3.5) by a function $w$ in a set $\mathcal{W}$ that is defined with respect to a given boundary component $\Gamma_{\text {shock }}^{\varepsilon, \delta}$ and depends on the given values $\rho_{0}, \rho_{1}$, and $\bar{\rho}^{\varepsilon, \delta}=\left(\bar{c}_{\rho_{0}}\right)^{-1}\left(r^{\varepsilon, \delta}\left(\theta_{w}\right)\right)$ :

Definition 3.2. The elements $w$ of $\mathcal{W} \subset C_{\left(-\gamma_{1}\right)}^{2}$ satisfy

(W1) $\rho_{0}<\bar{\rho}^{\varepsilon, \delta} \leq w \leq \rho_{1}, w=\rho_{1}$ on $\Gamma_{\text {sonic }}, w_{\nu}=0$ on $\Gamma_{0}$, and $w\left(P_{2}\right)=\bar{\rho}^{\varepsilon, \delta}$;

(W2) $\|w\|_{\alpha_{0}} \leq K_{0},\|w\|_{2+\alpha_{0}, \Omega_{\mathrm{loc}}} \leq K_{0}$, and $\|w\|_{1+\mu, \Gamma\left(d_{0}\right)} \leq K_{0}$

(W2) $\|w\|_{2}^{\left(-\gamma_{1}\right)} \leq K_{1}$.

The weighted Hölder space is defined by (3.8). The values of $\gamma_{1}, \alpha_{0}, \mu \in(0,1)$, and $d_{0}$, as well as the values of $K_{0}$ and $K_{1}$, will be specified later. Obviously, $\mathcal{W}$ is closed, bounded, and convex.

The quasilinear equation (3.11) and the oblique derivative boundary condition (3.5) are now replaced by the linear equation and linear oblique derivative boundary condition on $\Gamma_{\text {shock }}^{\varepsilon, \delta}:=\left\{(r(\theta), \theta): \theta_{w} \leq \theta \leq \theta_{1}\right\}$ :

$$
\begin{array}{ll}
L^{\varepsilon,+} u:=\sum_{i=1}^{2} D_{i}\left(a_{i i}^{\varepsilon}(P, w) D_{i} u\right)+b^{\varepsilon}(P, w) D_{r} u=0 & \text { in } \Omega, \\
M u:=\beta_{1}(P, w) D_{r} u+\beta_{2}(P, w) D_{\theta} u=0 & \text { on } \Gamma_{\text {shock }}^{\varepsilon, \delta},
\end{array}
$$

with given $r(\theta) \in \mathcal{K}^{\varepsilon, \delta} \subset C^{1+\alpha_{1}}\left(\left[\theta_{w}, \theta_{1}\right]\right) \cap C^{2}\left(\left(\theta_{w}, \theta_{1}\right)\right)$ and $w \in \mathcal{W}$, where the repeated indices are summed as usual. Because of the cut-off function $\zeta, L^{\varepsilon,+}$ is uniformly elliptic in $\Omega$ with the ellipticity ratio depending on the data and $\varepsilon$.

In this section, we demonstrate the key point that, thanks to the uniform distance between the sonic circle $C_{0}$ of the right state $(0)$ and $\Gamma_{\text {shock }}$, for a given function $w \in \mathcal{W}$, the solution $u$ to the linear equation (3.12) with the remaining boundary conditions:

$$
u=\rho_{1} \text { on } \Gamma_{\text {shock }}, \quad u_{\nu}=0 \text { on } \Gamma_{0}, \quad u\left(P_{2}\right)=\bar{\rho}^{\varepsilon, \delta},
$$

satisfies the Hölder and Schauder estimates in $\Omega^{\prime}$ and the uniform bound in $C^{1+\mu}\left(\Gamma\left(d_{0}\right)\right)$ near $\Gamma_{\text {shock }}^{\varepsilon, \delta}$ for any $\mu<\min \left\{\gamma_{1}, \alpha_{1}\right\}$. This bound gives rise to enough compactness to establish the existence of a solution to the quasilinear problem by applying the Schauder fixed point theorem.

First, we state the Schauder estimates up to the fixed boundary $\Gamma_{\text {sonic }}$ with the Dirichlet boundary condition and to $\Gamma_{0}$ with the Neumann boundary condition, and the Hölder estimates at the corners in $V^{\prime}$. 
Lemma 3.3. Assume that $\Gamma_{\text {shock }}$ is parameterized as $\{(r(\theta), \theta)\}$ with $r(\theta) \in \mathcal{K}^{\varepsilon, \delta}$ for some $\alpha_{1}$ and that $w \in \mathcal{W}$ for given $K_{0}, K_{1}, \alpha_{0}$, and $\gamma$. Then there exist $\gamma_{V}, \alpha_{\Omega} \in(0,1)$ such that any solution $u \in C_{l o c}^{2+\alpha_{\Omega}}\left(\Omega^{\prime}\right) \cap C^{\gamma_{V}}\left(\Omega_{V^{\prime}}\left(d_{0}\right)\right)$ to the linear problem (3.12)-(3.13) satisfies

$$
\|u\|_{\gamma, \Omega_{V^{\prime}}\left(d_{0}\right)} \leq C_{1}\|u\|_{0} \quad \text { for any } \gamma \leq \gamma_{V}
$$

and

$$
\|u\|_{2+\alpha, \Omega_{l o c}^{\prime}} \leq C_{2}\|u\|_{0} \quad \text { for any } \alpha \leq \alpha_{\Omega} .
$$

The exponent $\gamma_{V}$ depends on the data $\rho_{0}, \rho_{1}$, and $\theta_{w}$; and both $\alpha_{\Omega}$ and $\gamma_{V}$ depend on $\varepsilon$ but are independent of $\alpha_{1}$ and $\gamma_{1}$. The constant $C_{2}$ is independent of $K_{1}$ but depends on $K_{0}$.

Proof. The corner estimates at $P_{1}$ and $P_{3}$ directly follow from the results in Theorem 1 , Lieberman [17]. Near the origin, the wedge angle is larger than $\pi$; thanks to the symmetry of the governing equation in the $\theta$-axis with form (1.13), we derive the corner estimate as follows.

First we flat out the boundary by introducing the transformation:

$$
\left(r^{\prime}, \theta^{\prime}\right)=\left(r, \frac{\pi}{\pi-\theta_{w}}\left(\theta-\theta_{w}\right)\right), \quad\left(\xi^{\prime}, \eta^{\prime}\right)=\left(r^{\prime} \cos \theta^{\prime}, r^{\prime} \sin \theta^{\prime}\right) .
$$

Then the governing equation in the $\left(r^{\prime}, \theta^{\prime}\right)$-coordinates takes the form

$$
\begin{aligned}
\tilde{Q}^{\varepsilon,+} \rho= & \left(\left(\zeta\left(c^{2}(w)-r^{2}\right)+\varepsilon\right) \rho_{r}\right)_{r}+\left(\frac{\zeta\left(c^{2}(w)-r^{2}\right)+\varepsilon}{r}+r\right) \rho_{r} \\
& +\frac{\pi^{2}}{\left(\pi-\theta_{w}\right)^{2}}\left(\frac{c^{2}(w)+\varepsilon}{r^{2}} \rho_{\theta}\right)_{\theta},
\end{aligned}
$$

and

$$
\begin{aligned}
\tilde{Q}^{\varepsilon,+} \rho= & \left(\left(\left(\zeta\left(c^{2}(w)-r^{2}\right)+\varepsilon\right) \frac{\xi^{2}}{r^{2}}+\frac{\pi^{2}}{\left(\pi-\theta_{w}\right)^{2}} \frac{\left(c^{2}+\varepsilon\right) \eta^{2}}{r^{2}}\right) \rho_{\xi}\right)_{\xi} \\
& +\left(\left(\left(\zeta\left(c^{2}(w)-r^{2}\right)+\varepsilon\right) \frac{\xi \eta}{r^{2}}-\left(\frac{\pi^{2}}{\left(\pi-\theta_{w}\right)^{2}} \frac{\left(c^{2}+\varepsilon\right) \xi \eta}{r^{2}}\right) \rho_{\eta}\right)_{\xi}\right. \\
& +\left(\left(\left(\zeta\left(c^{2}(w)-r^{2}\right)+\varepsilon\right) \frac{\xi \eta}{r^{2}}-\frac{\pi^{2}}{\left(\pi-\theta_{w}\right)^{2}} \frac{\left(c^{2}+\varepsilon\right) \xi \eta}{r^{2}}\right) \rho_{\xi}\right)_{\eta} \\
& +\left(\left(\left(\zeta\left(c^{2}(w)-r^{2}\right)+\varepsilon\right) \frac{\eta^{2}}{r^{2}}+\frac{\pi^{2}}{\left(\pi-\theta_{w}\right)^{2}} \frac{\left(c^{2}+\varepsilon\right) \xi^{2}}{r^{2}}\right) \rho_{\eta}\right)_{\eta}+\xi \rho_{\xi}+\eta \rho_{\eta}
\end{aligned}
$$

in the $\left(\xi^{\prime}, \eta^{\prime}\right)$-coordinates, where we drop ' for simplicity without confusion. The eigenvalues of (3.16) are

$$
\lambda_{1}=\zeta\left(c^{2}-r^{2}\right)+\varepsilon, \quad \lambda_{2}=\left(\frac{\pi}{\pi-\theta_{w}}\right)^{2}\left(c^{2}+\varepsilon\right) .
$$

Note that the transformation from the $(\xi, \eta)$-coordinates to $\left(\xi^{\prime}, \eta^{\prime}\right)$-coordinates is

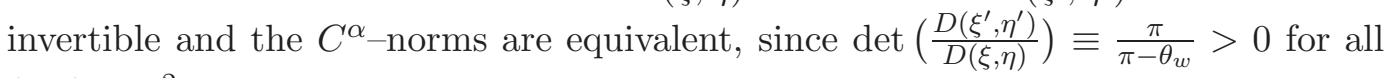
$(r, \theta) \in \mathbb{R}^{2}$.

As for the proof of the equivalence of the two norms, we have two cases: 


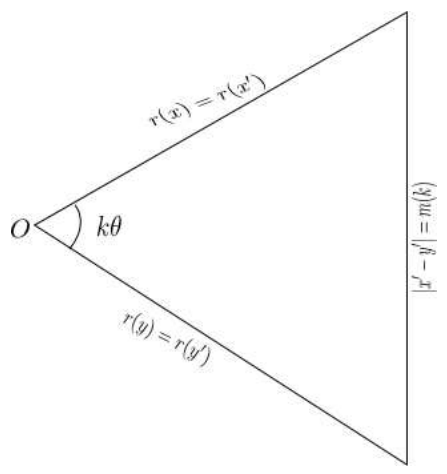

Figure 3. Scaling of the angles

Case 1. If $\theta \geq \frac{\pi}{2}$ as in Fig. 3, then

$$
\theta^{\prime}=k \theta \geq \frac{\pi}{2} \quad \text { with } k=\frac{\pi-\theta_{w}}{\pi} .
$$

Since $1<k<2,|x-y| \geq \max \{r(x), r(y)\}$ and $\left|x^{\prime}-y^{\prime}\right| \geq \max \left\{r\left(x^{\prime}\right), r\left(y^{\prime}\right)\right\}$. Then the equivalence of the two $C^{1,1}$-norms can be easily shown by setting $r(x)=r\left(x^{\prime}\right)$ and $r(y)=r\left(y^{\prime}\right)$.

Case 2. If $\theta<\frac{\pi}{2}$, then the distance between two points in the $(\xi, \eta)$-coordinates and $\left(\xi^{\prime}, \eta^{\prime}\right)$-coordinates is equivalent. By the cosine law, we define

$$
m(k):=\left|x^{\prime}-y^{\prime}\right|^{2}=r(x)^{2}+r(y)^{2}-2 r(x) r(y) \cos (k \theta),
$$

and then $\frac{\partial m(k)}{\partial k}=2 k r(x) r(y) \sin (k \theta)>0$. Thus,

$$
|x-y|=\sqrt{m(1)} \leq \sqrt{m(k)}=\left|x^{\prime}-y^{\prime}\right| \leq \sqrt{m(2)} \leq 2 \sqrt{m(1)}=2|x-y| .
$$

Therefore, we can obtain the Hölder estimate of the solution at $O$. Here $\gamma_{V}$ depends on the angle at the corner, a fixed value that depends on the data $\left(\rho_{0}, \rho_{1}, \theta_{w}\right)$, and the ellipticity ratio $\varepsilon$, but independent of $\gamma_{1}, \alpha_{1}, K_{0}$, and $K_{1}$.

Finally, we can use the standard interior and boundary Schauder estimates to obtain the local estimate (3.15). The constant $C_{2}$ depends on $\varepsilon$, the $C^{\alpha}$-norm of the coefficients $a_{i j}$, and the domain.

Because the interior Schauder estimates can be further applied, a solution in $C_{\text {loc }}^{2+\alpha}\left(\Omega^{\prime}\right)$ is actually in $C_{\mathrm{loc}}^{3}(\Omega)$.

We next establish the Hölder gradient estimates on $\Gamma_{\text {shock. }}$ It is at this point that we need to derive the basic estimates at point $P_{2}$ where the boundary operator $M$ is not oblique. In order to avoid handling the Neumann boundary condition on the wedge boundary $\theta=\theta_{w}$ separately at each step of this proof, we reflect $\Omega$ across the wedge boundary $\theta=\theta_{w}$, without further comment, i.e., $\Omega$ includes $\Sigma_{0}$, and let $\Gamma_{\text {shock }}$ stand for the full $C^{1+\alpha_{1}}$-boundary in Lemma 3.4 below. In addition, we extend $\tilde{u}\left(2 \theta_{w}-\theta\right)=u(\theta)$ for $\theta \in\left(\theta_{w}, \theta_{1}\right)$ in a small neighborhood of $\theta_{w}$. We still denote $\tilde{u}$ by $u$ for simplicity without confusion. 
Lemma 3.4. Assume that $\Gamma_{\text {shock }}$ is given by $\{(r(\theta), \theta)\}$ with $r(\theta) \in \mathcal{K}^{\varepsilon, \delta}$ for some $\alpha_{1}$ and that $w \in \mathcal{W}$ for given $K_{0}, K_{1}, \alpha_{0}$, and $\gamma_{1}$. Then there exists a positive constant $d_{0}$ such that, for any $d \leq d_{0}$, the solution $u \in C_{\text {loc }}^{1}\left(\Omega \cup \Gamma_{\text {shock }}\right) \cup C_{l o c}^{3}(\Omega)$ to the linear problem (3.12)-(3.13) satisfies

$$
\|u\|_{1+\mu, \Gamma(d) \backslash B_{d}\left(P_{1}\right)} \leq C\left(\varepsilon, \delta, \alpha_{1}, \gamma_{1}, K_{1}, d_{0}\right)\|u\|_{0}
$$

for any $\mu<\min \left\{\gamma_{1}, \alpha_{1}\right\}$.

Proof. Away from a neighborhood $B_{d_{0}}\left(P_{2}\right)$ of $P_{2}$, the operator $M$ is oblique. Thus we can apply Theorem 6.30 in [1] to obtain (3.17) in $\Gamma(d) \backslash\left\{B_{d_{0}}\left(P_{1}\right) \cup B_{d_{0}}\left(P_{2}\right)\right\}$, with a constant $C$ depending on $\epsilon, \alpha_{1}, \Omega, d_{0}$, and $K_{0}$. For the estimates near $P_{2}$, the proof is adopted from [4, which is similar. The main idea is that, for a given solution $u$ to (3.12)-(3.13), we define

$$
v=\frac{u}{1+\|D u\|_{0}} \quad \text { and } \quad z=M v=\sum_{i=1}^{2} \beta_{i}(P) D_{i} v .
$$

For $d_{0}>0$ small enough, $O \notin B_{d_{0}}\left(P_{2}\right)$. Then we construct barrier functions $\pm g$ for $z$ on $B:=B_{d_{0}}\left(P_{2}\right) \cap \bar{\Omega}$, by finding a suitable positive, increasing function $g, g(0)=0$, such that

$$
|z| \leq g
$$

More precisely, $g(\zeta)=g_{0} \zeta^{\mu}$ for any $\mu<\gamma_{2}$. This barrier function leads to

$$
|D(z+g)| \leq\|(z+g)\|_{1+\gamma_{2}}^{(1-\mu)} d^{\mu-1} \leq C(m) d^{\mu-1} \quad \text { for } d<d_{0},
$$

which leads to

$$
\|v\|_{1+\mu} \leq C \text {. }
$$

Finally, using the definition of $v$ in (3.18), we apply the interpolation inequality with small $\vartheta>0$ to obtain

$$
\|u\|_{1+\mu} \leq C\left(1+\|D u\|_{0}\right) \leq C\left(1+\vartheta\|u\|_{1+\mu}+C_{\vartheta}\|u\|_{0}\right)
$$

and thus (3.17) holds. Therefore, we obtain the Hölder gradient estimate at $\Gamma_{\text {shock }}$ for the solution $u$ of (3.12). See [4] for more details.

Now we focus on the existence of solutions in Theorem 3.1 for problem (3.12) (3.13). First we introduce two definitions with some modification in comparison with [13].

We say that problem (3.12) - (3.13) is locally solvable if, for each $y \in \bar{\Omega}$, there exists a neighborhood $O(y)$ such that, for any $h \in C(\bar{N})$ with $N:=O(y) \cap\left\{\bar{\Omega} \backslash\left(\left\{P_{2}\right\} \cup\right.\right.$ $\left.\left.\Gamma_{\text {sonic }}\right)\right\}$, there exists a solution $v \in C^{2}(N) \cap C(\bar{N})$ of the problem $L^{\varepsilon,+} v=0$ in $N \cap \Omega, M v=0$ on $N \cap \partial \Omega$, and $v=h$ on $\partial^{\prime} N$, when $P_{2} \notin \bar{N}(y)$; or $L^{\varepsilon,+} v=0$ in $N \cap \Omega, M v=0$ on $N \cap \partial \Omega, v=h$ on $\partial^{\prime} N$, and $\left.v\right|_{P_{2}}=\bar{\rho}^{\varepsilon, \delta}$, when $P_{2} \in \bar{N}(y)$. Here $\partial^{\prime} N=\partial N \cap \Omega$. For brevity, we denote this function $v$ by $(h)_{y}$ to emphasize its dependence on $h$ and $y$. 
A subsolution (supersolution) of (3.12)-(3.13) is a function $w \in C(\bar{\Omega})$ with

$$
w\left(r\left(\theta_{w}\right), \theta_{w}\right) \leq \bar{\rho}^{\varepsilon, \delta} \quad\left(w\left(r\left(\theta_{w}\right), \theta_{w}\right) \geq \bar{\rho}^{\varepsilon, \delta}\right)
$$

such that, for any $y \in \bar{\Omega}$, if $h \geq w(h \leq w)$ on $\partial^{\prime} N$, then $(h)_{y} \geq w\left((h)_{y} \leq w\right)$ in $N$. The set of all subsolutions (supersolutions) is denoted by $S^{-}\left(S^{+}\right)$.

We now establish the existence of solutions to problem (3.12)-(3.13).

Lemma 3.5. Assume that $\Gamma_{\text {shock }}$ is given by $\{(r(\theta), \theta)\}$ with $r(\theta) \in \mathcal{K}^{\varepsilon, \delta}$ for some $\alpha_{1}$ and that $w \in \mathcal{W}$ for given $K_{0}, K_{1}, \alpha_{0}$, and $\gamma_{1}$. Then there exist $\gamma_{V}, \alpha_{\Omega} \in(0,1)$ and $d_{0}>0$, which are independent of $\gamma_{1}$ and $\alpha_{1}$, such that there exists a solution

$$
u^{\varepsilon, \delta} \in C^{1+\mu}\left(\Gamma(d) \backslash B_{d}\left(P_{1}\right)\right) \cap C^{2+\alpha}\left(\Omega^{\prime}\right) \cap C^{\gamma}\left(\Omega_{V^{\prime}}(d)\right)
$$

to the linear problem (3.12) -3.13) for any $\alpha \leq \alpha_{\Omega}, \mu<\min \left\{\gamma_{1}, \alpha_{1}\right\}, \gamma \leq \gamma_{V}$, and $d \leq d_{0}$, which satisfies (3.14) (3.15) and (3.17).

Proof. For fixed $\varepsilon, \delta>0$, we denote $u^{\varepsilon, \delta}=u$ in the proof without confusion. We use the Perron method to show the existence of a solution to problem (3.12)-(3.13).

It suffices to show the local existence at $P_{2}$. In fact, let $B_{2}$ be a sufficiently small neighborhood of $P_{2}$ with smooth boundary such that $O \notin B_{2}, \beta_{1} \leq 0$, and $\beta_{2}<0$. Then we study the local existence in the $(\xi, \eta)$-coordinates in $B_{2}$. Reflect the region $B_{2}$ across $\theta=\theta_{w}$ to obtain a new region, which is still denoted by $B_{2}$. Then we introduce the coordinate transform in a neighborhood of $P_{2}$ :

$$
\hat{\xi}=\hat{\xi}(r, \theta), \quad \hat{\eta}=\hat{\eta}(r, \theta)
$$

such that

$$
\begin{array}{ll}
\hat{\xi}\left(r_{w}, \theta_{w}\right)=0, & \hat{\eta}\left(r_{w}, \theta_{w}\right)=0, \\
\frac{\partial(\hat{\xi}, \hat{\eta})}{\partial r}=(0,-1), & \frac{\partial(\hat{\xi}, \hat{\eta})}{\partial \theta}=-\left(\frac{1}{\beta_{2}}, \frac{\beta_{1}}{\beta_{2}}\right) .
\end{array}
$$

Let $\Gamma_{\text {shock }}:=\{(\hat{\xi}, \hat{\eta}): \hat{\eta}=f(\hat{\xi})\}=\{(r, \theta): r=r(\theta)\}$ in $\hat{B}_{2}$. Then $\hat{\eta}(r(\theta), \theta)=$ $f(\hat{\xi}(r(\theta), \theta))$ and hence $f^{\prime}(\hat{\xi})=-\left(\beta_{1}-\beta_{2} r^{\prime}(\theta)\right) \geq 0$, and the function $f(\hat{\xi})$ is increasing in $\hat{\xi}$ on $\Gamma_{\text {shock }} \cap \hat{B}_{2}$. Thus, from $\frac{\partial \hat{\xi}}{\partial \theta}=-\frac{1}{\beta_{2}}>0$ and $\frac{\partial \hat{\xi}}{\partial r}=0$, we have

$$
f(\hat{\xi}) \geq 0
$$

We replace $\Omega$ by $\Omega_{\sigma}$ which is the $\sigma$-distance from point $P_{2}$ upward, see Fig. 4 , On the bottom straight boundary of $\Omega_{\sigma}$, we impose

$$
u=\bar{\rho}^{\varepsilon, \delta} \quad \text { on the bottom of } \Omega_{\sigma} .
$$

Now we study the following boundary value problem:

$$
\begin{cases}\hat{L}^{\varepsilon, \delta} u=\sum_{i, j=1}^{2} \hat{a}_{i j} D_{j} u+\sum_{i=1}^{2} \hat{b}_{i} D_{i} u=0 & \text { in } \Omega_{\sigma}, \\ \hat{M} u=\partial_{\hat{\xi}} u=0 & \text { on } \partial \Omega_{\sigma} \cap \Gamma_{\text {shock }}, \\ u=h & \text { on } \partial B_{2} \cap \Omega, \\ u=\bar{\rho}^{\varepsilon, \delta} & \text { on } \Sigma_{\sigma},\end{cases}
$$




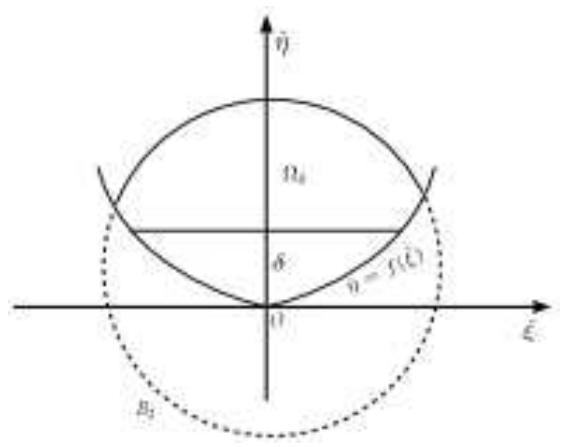

Figure 4. Domain with tip $P_{2}$ removed

where

$$
\begin{aligned}
& \tilde{a}_{11}^{\varepsilon}=\frac{\hat{a}_{11}^{\varepsilon}}{\hat{\beta}_{2}^{2}}, \quad \tilde{a}_{12}^{\varepsilon}=\tilde{a}_{21}^{\varepsilon}=-\frac{\hat{\beta}_{1}}{\hat{\beta}_{2}^{2}} \hat{a}_{22}^{\varepsilon}, \quad \tilde{a}_{22}^{\varepsilon}=\hat{a}_{11}^{\varepsilon}+\left(\frac{\hat{\beta}_{1}}{\hat{\beta}_{2}}\right)^{2} \hat{a}_{22}, \\
& \tilde{b}_{1}^{\varepsilon}=\frac{\partial \hat{a}_{11}^{\varepsilon}}{\partial \hat{\eta}}-\frac{\hat{a}_{22}^{\varepsilon}}{\hat{\beta}_{2}^{2}} \frac{\partial \hat{\beta}_{1}}{\partial \hat{\xi}}+\frac{\hat{\beta}_{1} \hat{a}_{22}^{\varepsilon}}{\hat{\beta}_{2}^{2}} \frac{\partial \hat{\beta}_{2}}{\partial \hat{\eta}}+\frac{\hat{\beta}_{1} \hat{a}_{22}^{\varepsilon}}{\hat{\beta}_{2}^{3}} \frac{\partial \hat{\beta}_{2}}{\partial \hat{\xi}}-\frac{\hat{\beta}_{1}^{2} \hat{a}_{22}^{\varepsilon}}{\hat{\beta}_{2}^{3}} \frac{\partial \hat{\beta}_{2}}{\partial \hat{\eta}}+\left(\frac{\hat{\beta}_{1}}{\hat{\beta}_{2}}\right)^{2} \frac{\partial \hat{a}_{22}^{\varepsilon}}{\partial \hat{\eta}}-\hat{b}^{\varepsilon}, \\
& \tilde{b}_{2}^{\varepsilon}=-\frac{\hat{a}_{22}^{\varepsilon}}{\hat{\beta}_{2}^{3}} \frac{\partial \hat{\beta}_{2}}{\partial \hat{\xi}}+\frac{\hat{a}_{22}^{\varepsilon} \hat{\beta}_{1}}{\hat{\beta}_{2}^{3}}+\frac{1}{\hat{\beta}^{2}} \frac{\partial \hat{a}_{22}^{\varepsilon}}{\partial \hat{\xi}}-\frac{\hat{\beta}_{1}}{\hat{\beta}_{2}^{2}} \frac{\partial \hat{a}_{22}^{\varepsilon}}{\partial \hat{\eta}} .
\end{aligned}
$$

Here $\hat{a}_{i i}^{\varepsilon}, \hat{b}^{\varepsilon}$, and $\hat{\beta}_{i}, i=1,2$, are the coefficients of (3.12)-(3.13) in the $(\hat{\xi}, \hat{\eta})$-coordinates, and $h$ is a continuous function satisfying $\bar{\rho}^{\varepsilon, \delta}<h \leq \rho_{1}$. Following Lieberman [13], there exists a solution

$$
u_{\sigma} \in C\left(\bar{\Omega} \cap \overline{\hat{B}_{2}}\right) \cup C^{2, \alpha}\left(\Omega_{\sigma} \cap \hat{B}_{2}\right)
$$

for small enough $\hat{B}_{2}$. The maximum principle holds for $u_{\sigma}$, which converges locally in $C^{2}\left(\Omega \cap \hat{B}_{2}\right)$ to a solution in $C^{2+\alpha}\left(\Omega \cap \hat{B}_{2}\right)$ as $\sigma \rightarrow 0_{+}$.

We now use a barrier function to obtain the continuity of $u$ at $P_{2}$. We consider the auxiliary function

$$
v=\bar{\rho}^{\varepsilon, \delta}+c\left(1-e^{-l \hat{\eta}}\right),
$$

where $c>0$ and $l>0$ are specified later. For the oblique derivative boundary condition along $\Omega_{\sigma} \cap \Gamma_{\text {shock }}$, we have the following two cases:

Case 1: $\tilde{\beta} \cdot \boldsymbol{\nu}>0$ and $\tilde{M}\left(v-\bar{\rho}^{\varepsilon, \delta}\right) \geq 0$ when $\hat{\xi}>0$,

Case 2: $\tilde{\beta} \cdot \boldsymbol{\nu}<0$ and $\tilde{M}\left(v-\bar{\rho}^{\varepsilon, \delta}\right) \leq 0$ when $\hat{\xi}<0$,

where $\boldsymbol{\nu}$ denotes the outward normal to $\Omega_{\sigma}$ at $\Omega_{\sigma} \cap \Gamma_{\text {shock}}$.

Moreover, it is easy to see that $v \geq \bar{\rho}^{\varepsilon, \delta}$ on $\Sigma_{\sigma}$. Choose $C$ large enough such that $v \geq \sup |h|$ on $\partial \hat{B}_{2} \cap \Omega$. For the equation, we have

$$
\tilde{a}_{i j}^{\varepsilon} D_{j} v+\tilde{b}_{i}^{\varepsilon} D_{i} v=-c e^{-l \hat{\eta}}\left(l^{2} \tilde{a}_{11}^{\varepsilon}-l \tilde{b}_{1}^{\varepsilon}\right),
$$


which is less than a negative constant by choosing $l>\frac{\left\|\tilde{b}^{\varepsilon}\right\|_{0}}{\lambda}$, where $\lambda \leq \tilde{a}_{11}^{\varepsilon}(\hat{\xi}, \hat{\eta})$. Thus,

$$
\bar{\rho}^{\varepsilon, \delta} \leq u \leq v .
$$

Then $u$ is continuous at point $P_{2}$. The continuity of $u$ at the other points follows from Lieberman's argument in [15, 17].

By Lemma 3.4, we have $u \in C^{1, \mu}\left(\hat{B}_{2} \cap \bar{\Omega}\right)$.

In order to establish the global existence of solutions, it is required to show

$$
\sup _{\Omega}\left(w^{-}-w^{+}\right)=0
$$

where $w^{ \pm}$are the supersolution and subsolution of problem (3.12)-(3.13), respectively.

In fact, we set $m:=\sup _{\Omega}\left(w^{-}-w^{+}\right)$. We assume that $m>0$ in $\Omega$. Since $w^{-}\left(P_{2}\right)-$ $w^{+}\left(P_{2}\right) \leq 0$, there exists a neighborhood $\hat{B}_{2}\left(P_{2}\right)$ of $P_{2}$ such that $w^{-}(y)-w^{+}(y)<m$ for $y \in \hat{B}_{2}\left(P_{2}\right)$. Now we define

$$
\mathcal{Y}:=\left\{y \in \bar{\Omega}: w^{-}(y)-w^{+}(y)=m\right\} .
$$

Let $y_{0} \in \mathcal{Y}$ such that

$$
\operatorname{dist}\left(y_{0}, P_{2}\right)=\min _{y \in \mathcal{Y}} \operatorname{dist}\left(y, P_{2}\right) .
$$

Let $\bar{w}^{ \pm}$be the lifts of $w^{ \pm}$in $M\left(y_{0}\right)$. We see that $\bar{w}^{-}-\bar{w}^{+} \leq m$ on $\partial^{\prime} N$. The strong maximum principle implies that either $\bar{w}^{-}-\bar{w}^{+}<m$ in $M$ or $\bar{w}^{-}-\bar{w}^{+} \equiv m$. Since $\bar{w}^{-}\left(y_{0}\right)-\bar{w}^{+}\left(y_{0}\right) \geq w^{-}\left(y_{0}\right)-w^{+}\left(y_{0}\right)=m$, it follows that $\bar{w}^{-}-\bar{w}^{+}=m$ in $N$, and hence

$$
\bar{w}^{-}-\bar{w}^{+} \equiv m \quad \text { on } \partial^{\prime} N,
$$

which contains the point of $\mathcal{Y}$ closer to $P_{2}$ than $y_{0}$. This is a contradiction with the definition of $y_{0}$.

We refer to Lieberman [15] for handling the mixed case and both points $P_{1}$ and $P_{3}$. As for the interior and the Dirichlet boundary condition on the sonic $\operatorname{arc} \Gamma_{\text {sonic }}$, they are classical since the equation is uniformly elliptic for fixed $\varepsilon>0$ (see GilbargTrudinger [1]).

With all of these, we then employ the Perron method to establish the existence of a global solution.

3.3. Proof of Theorem 3.1; Regularized Nonlinear Fixed Boundary Problem. We now establish the existence of solutions to the nonlinear problem (3.2) with a fixed boundary.

Lemma 3.6. For $\varepsilon \in\left(0, \varepsilon_{0}\right)$ and $\delta \in\left(0, \delta_{0}\right)$, given $r(\theta) \in \mathcal{K}^{\varepsilon, \delta} \subset C^{1+\alpha_{1}}$, there exists a solution $\rho^{\varepsilon, \delta} \in C_{\left(-\gamma_{1}\right)}^{2+\alpha}\left(\Omega^{\varepsilon, \delta}\right)$ to problem (3.2) and (3.6) -(3.7) with the oblique derivative condition $M \rho^{\varepsilon, \delta}=0$ for some $\alpha(\varepsilon, \delta), \gamma_{1}(\varepsilon, \delta) \in(0,1)$ such that

$$
\rho_{0}<\bar{\rho}^{\varepsilon, \delta} \leq \rho^{\varepsilon, \delta} \leq \rho_{1} \text {. }
$$


Moreover, for some $d_{0}>0$, the solution $\rho^{\varepsilon, \delta}$ satisfies

$$
\left\|\rho^{\varepsilon, \delta}\right\|_{\gamma, \Gamma\left(d_{0}\right) \cup B_{d_{0}}\left(P_{1}\right)} \leq K_{2},
$$

where $\gamma$ and $K_{2}$ depend on $\delta, \varepsilon, \gamma_{V}$, and $K_{1}$, but are independent of $\alpha_{1}$. In addition, the solutions satisfy the following three properties:

(i) Ellipticity of Eq. (3.2) : $c^{2}\left(\rho^{\varepsilon, \delta}\right)-r^{2} \geq 0$ in $\bar{\Omega}^{\varepsilon, \delta}$;

(ii) (3.1) can always be integrated: $\bar{c}\left(\rho^{\varepsilon, \delta}, \rho_{0}\right)-r \leq 0$ on $\Gamma_{\text {shock }}^{\varepsilon, \delta}$;

(iii) Local behaviour of the density near $\Gamma_{\text {shock }}^{\varepsilon, \delta}$ and the convexity of $\Gamma_{\text {shock }}^{\varepsilon, \delta} \rho^{\varepsilon, \delta}$ is monotone on $\Gamma_{\text {shock }}^{\varepsilon, \delta}$.

The idea is to employ the fixed point theorem to prove the existence of the solution and then to find the barrier function to control the behaviour of the solution. The proof is long and similar to the one in [4] or [12, while the main difference here is the singularity at origin for which we need to treat. Thus, we postpone the detailed proof which can be found in Appendix for self-containedness.

\subsection{Proof of Theorem 3.1; The Regularized Nonlinear Free Boundary}

Problem. We now show the existence of a solution to the regularized free boundary problem.

Lemma 3.7. For each $\varepsilon \in\left(0, \varepsilon_{0}\right)$ and $\delta \in\left(0, \delta_{0}\right)$ with some $\varepsilon_{0}>0$ and $\delta_{0}>0$, there exists a solution $\left(\rho^{\varepsilon, \delta}, r^{\varepsilon, \delta}\right) \in C_{(-\gamma)}^{2+\alpha}\left(\Omega^{\varepsilon, \delta}\right) \times C^{1+\alpha_{1}}\left(\left[-\frac{\pi}{2}, \theta_{1}\right)\right)$ to the regularized free boundary problem (3.2)-(3.7).

Proof. For the notational simplicity, we suppress the $(\varepsilon, \delta)$-dependence in the proof.

For each $r(\theta) \in \mathcal{K}^{\varepsilon, \delta} \subset C^{1+\alpha_{1}}\left(\left[\theta_{w}, \theta_{1}\right]\right)$, using the solution $\rho$ of the nonlinear fixed boundary problem (3.2) and (3.6) -(3.7) given by Lemma 3.5, we define the map $J$ on $\mathcal{K}, \tilde{r}=J r$, as in (3.1):

$$
\tilde{r}(\theta)=r_{1}+\int_{\theta_{1}}^{\theta} g(r(s), s, \rho(r(s), s)) d s .
$$

There are two cases for the approximate shock position $\tilde{r}(\theta)$ :

Case 1: $\tilde{r}\left(\theta_{w}\right) \geq c\left(\rho_{0}\right)+\delta$. We check that $J$ maps $\mathcal{K}$ into itself. It is easy to check that $\tilde{r}(\theta) \in C^{1+\gamma_{V}}\left(\left[\theta_{w}, \theta_{1}\right]\right) \cap C^{1+1}\left(\left[\theta_{w}, \theta_{1}\right)\right)$, from the definition of $\tilde{r}$ and by using Lemmas 3.3 3.4 and property $\left(K_{1}\right)$ follows from (3.26). By the definition of $g$ and $\rho\left(P_{2}\right)=\bar{\rho}, \tilde{r}^{\prime}(\theta)=0$ holds, which implies property $\left(K_{2}\right)$. Then it suffices to show that property $\left(K_{4}\right)$ holds, since the upper and lower bounds of $\rho$, Lemma 3.6, and $\left(K_{4}\right)$ imply $\left(K_{3}\right)$. From the expression of $g(r(\theta), \theta, \rho(r(\theta), \theta))$ and the upper and lower bounds of $\rho$, we have $\left(K_{4}\right)$.

Case 2: $\tilde{r}\left(\theta_{w}\right)<c\left(\rho_{0}\right)+\delta$. Since $\tilde{r}^{\prime}(\theta)>0$ for $\theta \in\left(\theta_{w}, \theta_{1}\right)$ and $r_{1}=c\left(\rho_{1}\right)>$ $c\left(\rho_{0}\right)+\delta$, there exists a unique $\theta_{a} \in\left(\theta_{w}, \theta_{1}\right)$ such that $\tilde{r}\left(\theta_{a}\right)=c\left(\rho_{0}\right)+\delta$. Now, choosing $\tau$ to be determined later such that $\tilde{r}\left(\theta_{a}+\tau\right) \leq c\left(\rho_{0}\right)+2 \delta$ and letting $x_{1}=\theta_{a}+\tau-\theta_{w}$, we modify the approximate shock position on $\theta_{w} \leq \theta \leq \theta_{a}+\tau$ by defining

$$
\hat{r}(\theta)=c\left(\rho_{0}\right)+\delta+A\left(\theta-\theta_{w}\right)^{3}+B\left(\theta-\theta_{w}\right)^{n}
$$


with

$$
A=\frac{1}{(n-3) x_{1}^{3}}\left(n a-b x_{1}\right), \quad B=\frac{1}{(n-3) x_{1}^{n}}\left(b x_{1}-3 a\right),
$$

where $a=\tilde{r}\left(\theta_{a}+\tau\right)-c\left(\rho_{0}\right)-\delta$ and $b=\tilde{r}^{\prime}\left(\theta_{a}+\tau\right)$.

Choose $\tau$ small enough such that

$$
b x_{1}-3 a>0,
$$

and then $n$ sufficiently large such that

$$
n a-b x_{1}>0,
$$

where $n$ depends on $\delta$, but independent of the iteration. Next, we choose $n$ and $\tau$ precisely. In fact, it is easy to see that

$$
|b| \leq \frac{c\left(\rho_{1}\right)}{c\left(\rho_{0}\right)} \sqrt{c^{2}\left(\rho_{1}\right)-c^{2}\left(\rho_{0}\right)},
$$

and

$$
\left|b x_{1}\right| \leq C\left(\rho_{0}, \rho_{1}, \theta_{1}, \theta_{w}\right):=\frac{c\left(\rho_{1}\right)}{c\left(\rho_{0}\right)} \sqrt{c^{2}\left(\rho_{1}\right)-c^{2}\left(\rho_{0}\right)}\left(\theta_{1}-\theta_{w}\right)
$$

If $3 \delta \leq b x_{1}$, we choose $\tau$ such that $a=\delta$ and $n_{1}=\frac{C\left(\rho_{0}, \rho_{1}, \theta_{1}, \theta_{w}\right)}{\delta}+1$, which depend only on $\delta, \rho_{0}$, and $\rho_{1}$.

If $3 \delta>b x_{1}$, letting $\tau$ small enough, we can obtain new $a$ and $b$ satisfying $3 a=b x_{1}$, where we choose the biggest $\tau$ smaller than the old one such that $3 a=b x_{1}$ holds. Note that $b x_{1}>0$ and $\tilde{r}\left(\theta_{a}\right)=c\left(\rho_{0}\right)+\delta$. Thus, choosing $n_{2}=4$, we have $A>0$ and $B=0$.

Let $n=\max \left(n_{1}, n_{2}\right)=n\left(\rho_{0}, \rho_{1}, \theta_{1}, \theta_{w}, \delta\right)$, which is independent of the iteration process. Thus, $\hat{r}(\theta)$, uniquely determined, is a strictly increasing function on $\left[\theta_{w}, \theta_{a}+\right.$ $\tau]$. Furthermore, we have

$$
0=\hat{r}^{\prime}\left(-\frac{\pi}{2}\right) \leq \hat{r}^{\prime}(\theta) \leq \hat{r}^{\prime}\left(\theta_{a}+\tau\right)=\tilde{r}^{\prime}\left(\theta_{a}+\tau\right) .
$$

We define

$$
\operatorname{Jr}(\theta)= \begin{cases}\tilde{r}(\theta) & \text { for } \theta \in\left[\theta_{a}+\tau, \theta_{1}\right], \\ \hat{r}(\theta) & \text { for } \theta \in\left[\theta_{w}, \theta_{a}+\tau\right] .\end{cases}
$$

It is easy to show that $\operatorname{Jr}(\theta), \theta \in\left[\theta_{w}, \theta_{1}\right]$, satisfies properties $\left(K_{1}\right)-\left(K_{4}\right)$.

First, since $\tilde{r}(\theta) \in C^{1+\gamma_{V}}\left(\left[\theta_{a}+\tau, \theta_{1}\right]\right), \hat{r}(\theta) \in C^{\infty}\left(\left[\theta_{w}, \theta_{a}+\tau\right]\right)$, and $(J r)^{\prime}(\theta) \in$ $C\left(\left[\theta_{w}, \theta_{1}\right]\right)$, we have

$$
\operatorname{Jr}(\theta) \in C^{1+\gamma_{V}}\left(\left[\theta_{w}, \theta_{1}\right]\right) .
$$

Next, for $\theta \in\left[\theta_{w}, \theta_{a}+\tau\right], \hat{r}^{\prime}(\theta)=3 A\left(\theta-\theta_{w}\right)^{2}+n B\left(\theta-\theta_{w}\right)^{n-1}$. Then

$$
\begin{aligned}
& \hat{r}^{\prime}\left(\theta_{2}\right)-\hat{r}^{\prime}\left(\theta_{3}\right) \\
& =3 A\left(\theta_{2}-\theta_{w}\right)^{2}-3 A\left(\theta_{3}-\theta_{w}\right)^{2}+n B\left(\theta_{2}-\theta_{w}\right)^{n-1}-n B\left(\theta_{3}-\theta_{w}\right)^{n-1} \\
& =3 A\left(\theta_{2}-\theta_{3}\right)\left(\theta_{2}+\theta_{3}-2 \theta_{w}\right)+n B\left(\theta_{2}-\theta_{3}\right)\left(\sum_{j=0}^{n-2} C_{n-2}^{j}\left(\theta_{2}-\theta_{w}\right)^{n-2-j}\left(\theta_{3}-\theta_{w}\right)^{j}\right) .
\end{aligned}
$$


Using the fact that $\theta_{2}-\theta_{w}, \theta_{3}-\theta_{w} \leq x_{1}$, and $A, B \geq 0$, we obtain

$$
\begin{aligned}
\left|\hat{r}^{\prime}\left(\theta_{2}\right)-\hat{r}^{\prime}\left(\theta_{3}\right)\right| & \leq\left|\theta_{2}-\theta_{3}\right|^{\alpha}\left(6 A x_{1}^{2-\alpha}+C(n) B x_{1}^{n-1-\alpha}\right) \\
& \leq C(n)\left(a x_{1}^{-1-\alpha}+b x_{1}^{-\alpha}\right)\left|\theta_{2}-\theta_{3}\right|^{\alpha} .
\end{aligned}
$$

Notice that $\tilde{r}^{\prime}=\frac{r}{\bar{c}} \sqrt{r^{2}-\bar{c}^{2}}, r \in C^{1+\gamma_{V}}$, and $\theta_{a}+\tau$ is uniformly away from $\theta_{1}$, which means that $\rho \in C^{1+\mu}$. We obtain

$$
\tilde{r}^{\prime} \leq C\left(\rho_{0}, \rho_{1}, \varepsilon, \delta\right) x_{1}^{1 / 2}
$$

which implies

$$
\left|\hat{r}^{\prime}\left(\theta_{2}\right)-\hat{r}^{\prime}\left(\theta_{3}\right)\right| \leq C\left(\rho_{0}, \rho_{1}, \varepsilon, \delta\right)\left|\theta_{2}-\theta_{3}\right|^{\alpha} \quad \text { if } \alpha \leq \frac{1}{2} .
$$

Thus

$$
\|J r\|_{C^{1+\alpha}\left(\left[-\frac{\pi}{2}, \theta_{1}\right]\right)} \leq C\left(\rho_{1}, \rho_{2}, \varepsilon, \delta\right)
$$

if $\alpha \leq \min \left\{\gamma_{V}, \frac{1}{2}\right\}$, which satisfies $\left(K_{1}\right)-\left(K_{4}\right)$.

Thus, we define a map

$$
J: \mathcal{K}^{\varepsilon, \delta} \rightarrow \mathcal{K}^{\varepsilon, \delta}
$$

by

$$
\tilde{r}=J r .
$$

Obviously, $\mathcal{K}^{\varepsilon, \delta}$ is a convex and closed subset of the Banach space $C^{\alpha_{1}}$, and $J$ is compact, if $\alpha_{1}<\min \left\{\gamma_{V}, \frac{1}{2}\right\}$. In order to use the Schauder fixed point theorem, we need to prove that $J$ is continuous on $\mathcal{K}^{\varepsilon, \delta}$.

Assume that $r_{m}, r \in \mathcal{K}^{\varepsilon, \delta}$ for $m=1,2, \cdots, r_{m} \rightarrow r$ as $m \rightarrow \infty$, and $\rho_{m}$ solves the fixed boundary problem for $r_{m}$ for each fixed $m$. Then, by the standard argument as in [4], $\rho_{m} \rightarrow \rho$, which solves the problem for $r$. Therefore, we have

$$
g\left(r_{m}(\theta), \theta, \rho_{m}\left(r_{m}(\theta), \theta\right)\right) \longrightarrow(r(\theta), \theta, \rho(r(\theta), \theta)) \quad m \rightarrow \infty,
$$

which implies $J r_{m} \rightarrow J r$ as $m \rightarrow \infty$ at the point where (3.3) holds for both $r_{m}$ and $r$. Then $J r_{m} \rightarrow J r$ as $m \rightarrow \infty$, if $J r$ belongs to Case 1. For Case 2, due to the construction, we divide it into three subcases:

$$
3 \delta<b x_{1} ; \quad 3 \delta>b x_{1} ; \quad 3 \delta=b x_{1},
$$

where $b=\tilde{r}^{\prime}\left(\theta_{a}+\tau\right), x_{1}=\theta_{a}+\tau-\theta_{w}$, and $\tilde{r}\left(\theta_{a}+\tau\right)=c\left(\rho_{0}\right)+2 \delta$ depend only on $r$ and $\delta$. For any case, it is easy to deduce that

$$
\left(\tau_{m}, \theta_{a, m}\right) \rightarrow\left(\tau, \theta_{a}\right), \quad\left(A_{m}, B_{m}\right) \rightarrow(A, B) \quad \text { when } m \rightarrow \infty .
$$

Then $J r_{m} \rightarrow J r$, with the fact that

$$
J r_{m}=c\left(\rho_{0}\right)+\delta+A_{m}\left(\theta-\theta_{w}\right)^{3}+B_{m}\left(\theta-\theta_{w}\right)^{n}
$$

for $\theta<\theta_{a, m}+\tau_{m}$, where $n, \theta_{w}$, and $\rho_{0}$ are universal constants.

Then, for any fixed $\varepsilon, \delta>0$, we obtain the existence of a solution $\left(\rho^{\varepsilon, \delta}, r^{\varepsilon, \delta}\right)$ to the free boundary problem by the standard fixed point argument. Moreover, we have $r^{\varepsilon, \delta} \in C^{1+\alpha}\left(\left[\theta_{w}, \theta_{1}\right]\right)$ for $\alpha \leq \alpha_{1}$. This completes the proof. 
3.5. Proof of Theorem 3.1; Completion. We note that Lemma 3.7 implies that there exists a solution $\left(\rho^{\varepsilon, \delta}, r^{\varepsilon, \delta}\right)$ such that $r^{\varepsilon, \delta} \in \mathcal{K}^{\varepsilon, \delta}$. From Lemma 3.6 and the interior Schauder estimate, we note that $\left\|\rho^{\varepsilon, \delta}\right\|_{C_{l o c}^{2, \alpha}} \leq C$, and $\rho^{\varepsilon, \delta}$ satisfies property (3.9). By Lemma 3.6, we have $c^{2}\left(\rho^{\varepsilon, \delta}\right) \geq r^{2}$. This completes the proof.

\section{Proof of Theorem 2.1; Existence of Solutions}

In this section, we study the limiting solution, as the elliptic regularization parameter $\varepsilon$ and the oblique derivative boundary regularization parameter $\delta$ tend to 0 . We start with the regularized solutions of problem (3.2) and (3.5)-(3.7), whose existence is guaranteed by Theorem 3.1. Denote by $\rho^{\varepsilon, \delta}$ a sequence of the regularized solutions of the boundary value problem.

For the solutions of the regularized problems, we first construct a uniform lower barrier to obtain the uniform ellipticity in any compact domain contained by $\bar{\Omega} \backslash \Gamma_{\text {sonic }}$, and also away from the points in $\Gamma_{\text {shock }}$ where their distance to the sonic circle of right state $C_{0}$ goes to zero as $\delta$ tends to zero.

Lemma 4.1. There exists a positive function $\varphi$, independent of $\varepsilon$ and $\delta$, such that

$$
c^{2}\left(\rho^{\varepsilon, \delta}\right)-\left(\xi^{2}+\eta^{2}\right) \geq \varphi \quad \text { in } \bar{\Omega} \backslash \Gamma_{\text {sonic }},
$$

and

$$
\varphi \rightarrow 0
$$

as $\operatorname{dist}\left((\xi, \eta), \Gamma_{\text {sonic }}\right) \rightarrow 0$, or $\max \left\{\operatorname{dist}\left((\xi, \eta), \Gamma_{\text {shock }}\right)\right.$, $\left.\operatorname{dist}\left((\xi, \eta), C_{0}\right)\right\} \rightarrow 0$.

Proof. For $0<R<1$ and $X_{0}=\left(\xi_{0}, \eta_{0}\right) \in \tilde{\Omega}$, as in [4], let

$$
\zeta(X)=1-\frac{\left(\xi-\xi_{0}\right)^{2}+\left(\eta-\eta_{0}\right)^{2}}{R^{2}} \quad \text { for } B_{R}\left(X_{0}\right) \cap \Gamma_{\text {sonic }}=\emptyset .
$$

We define

$$
\varphi(\xi, \eta)=\delta_{0}(\zeta(X))^{\tau}
$$

where $\delta_{0}$ and $\tau$ are two positive constants to be specified later, and $0 \leq \zeta \leq 1$ independent of $\varepsilon$. Then, piecing together these $B_{\frac{3}{4} R_{X_{0}}}\left(X_{0}\right), X_{0} \in \bar{\Omega} \backslash \Gamma_{\text {sonic }}$, we can obtain a local uniform lower barrier of $c^{2}\left(\rho^{\varepsilon, \delta}\right)-\left(\xi^{2}+\eta^{2}\right)$. That is,

$$
c^{2}\left(\rho^{\varepsilon, \delta}\right)-\xi^{2}-\eta^{2} \geq \varphi=\delta_{0} \zeta^{\tau} \quad \text { in } B_{\frac{3}{4} R_{X_{0}}}\left(X_{0}\right) \cap \overline{\Omega_{\varepsilon}}
$$

where $\delta_{0}$ and $\tau$ are independent of $\varepsilon$ (though they may depend on $R$ ). Moreover, $\delta_{0}$ tends to 0 as $\operatorname{dist}\left((\xi, \eta), \Gamma_{\text {sonic }}\right) \rightarrow 0$, or $\max \left\{\operatorname{dist}\left((\xi, \eta), \Gamma_{\text {shock }}\right), \operatorname{dist}\left((\xi, \eta), C_{0}\right)\right\} \rightarrow$ 0 , so does $\varphi$. See [4] for more details.

The proof of Lemma 4.1 also implies that we can obtain the uniform ellipticity of (3.2), which is independent of $\varepsilon$ in $B_{\frac{3}{4}} R_{X_{0}}\left(X_{0}\right) \cap \overline{\Omega_{\varepsilon}}$.

The uniform lower bound of $c^{2}-\xi^{2}-\eta^{2}$ independent of $\varepsilon$ implies that the governing equation (3.2) is locally uniformly elliptic, independent of $\varepsilon$ and $\delta$, which allows us to apply the standard local compactness arguments to obtain the limit $\rho$ locally in the interior of the domain. 
We first consider the behaviour of shock position $r^{\varepsilon, \delta}$, as $\varepsilon$ and $\delta$ tend to 0 . We divide the shock position into three cases:

Case 1: $c\left(\rho_{0}\right)<r(\theta) \leq \bar{c}\left(\rho_{1}, \rho_{0}\right)$ for all $\theta \in\left[\theta_{w}, \theta_{1}\right)$ and $r^{\prime}(\theta)=r \sqrt{\frac{r^{2}-\bar{c}^{2}}{\bar{c}^{2}}}$;

Case 2: $r\left(\theta_{w}\right)=c\left(\rho_{0}\right)$ and $c\left(\rho_{0}\right)<r(\theta) \leq \bar{c}\left(\rho_{1}, \rho_{0}\right), r^{\prime}(\theta)=r \sqrt{\frac{r^{2}-\bar{c}^{2}}{\bar{c}^{2}}}$ for all $\theta \in\left(\theta_{w}, \theta_{1}\right)$

Case 3: There exists $\theta_{a} \in\left(\theta_{w}, \theta_{1}\right)$ such that $r(\theta) \equiv c\left(\rho_{0}\right)$ for $\theta \in\left[\theta_{w}, \theta_{a}\right], r(\theta)>$ $c\left(\rho_{0}\right)$, and $r^{\prime}(\theta)=r \sqrt{\frac{r^{2}-\bar{c}^{2}}{\bar{c}^{2}}}$ for $\theta \in\left(\theta_{a}, \theta_{1}\right)$.

Lemma 4.2. There exist functions $r(\theta) \in C^{1}\left(\left[\theta_{w}, \theta_{1}\right]\right)$ and $\rho \in C_{l o c}^{2+\alpha}(\Omega) \cap C(\bar{\Omega})$, satisfying one of the three cases stated above, such that

$$
r^{\varepsilon, \delta} \rightarrow r \quad \text { in } C\left(\left[\theta_{w}, \theta_{1}\right]\right), \quad \rho^{\varepsilon, \delta} \rightarrow \rho \quad \text { in } \quad C_{l o c}^{2+\alpha},
$$

and $(\rho, r)$ is a solution of the free boundary problem (3.2)-(3.7).

Proof. For $\varepsilon, \delta>0$, it follows from Lemma 3.7 that

$$
r^{\varepsilon, \delta} \in C^{1+\alpha}\left(\left[\theta_{w}, \theta_{1}\right]\right), \quad\left\|r^{\varepsilon, \delta}\right\|_{C^{1}\left(\left[\theta_{w}, \theta_{1}\right]\right)} \leq C,
$$

where $C$ is independent of $\varepsilon$ and $\delta$. Thus, by the Ascoli-Arzela theorem, there exists a subsequence converging uniformly to a function $r(\theta)$ in $C^{\alpha}\left(\left[\theta_{w}, \theta_{1}\right]\right)$ as $\varepsilon, \delta \rightarrow 0$ for any $\alpha<1$. By the local ellipticity ( $c f$. Lemma 4.1) and the standard interior Schauder estimate, there exists a function $\rho \in C_{l o c}^{2+\alpha}$ such that $\rho^{\varepsilon, \delta} \rightarrow \rho$ in any compact subset contained by $\bar{\Omega} \backslash\left(\Gamma_{\text {sonic }} \cup \Gamma_{\text {shock }}\right)$, satisfying $Q \rho=0$ in $\Omega$.

For $\left(r\left(\theta_{0}\right), \theta_{0}\right) \in \Gamma_{\text {sock }}$ with $r\left(\theta_{0}\right)>c\left(\rho_{0}\right)$, there exist a neighborhood of $\theta_{0}$ and a constant $\delta^{\star}>0$ independent of $\varepsilon$ and $\delta$ such that $r^{\varepsilon, \delta} \geq c\left(\rho_{0}\right)+\delta^{\star}$ for $\varepsilon$ and $\delta$ small enough. It follows from $c\left(\rho^{\varepsilon, \delta}\right) \geq r^{\varepsilon, \delta} \geq c\left(\rho_{0}\right)+\delta^{\star}$ that

$$
\rho^{\varepsilon, \delta}>\rho_{0}+\delta^{\star} \text {. }
$$

Thus, we obtain the uniform ellipticity locally, as well as the uniform negativity of $\beta \cdot \boldsymbol{\nu}$ locally. Hence, we can pass the limit to obtain $\rho \in C^{1+\alpha}$ and

$$
M \rho=0 \quad \text { on } \Gamma_{\text {shock }} \text { near }\left(r\left(\theta_{0}\right), \theta_{0}\right)
$$

such that $r^{\prime}(\theta)=\frac{r}{\bar{c}} \sqrt{r^{2}-\bar{c}^{2}}$.

Then the remainder is to show the case that $\left(r\left(\theta_{0}\right), \theta_{0}\right) \in \Gamma_{\text {shock }}$ and $r\left(\theta_{0}\right)=c\left(\rho_{0}\right)$.

First, it follows from Lemma 3.6 that

$$
c\left(\rho_{0}\right) \leq r^{\varepsilon, \delta}(\theta) \leq c\left(\rho^{\varepsilon, \delta}\left(r^{\varepsilon, \delta}(\theta), \theta\right)\right) \leq c\left(\rho^{\varepsilon, \delta}\left(r^{\varepsilon, \delta}\left(\theta_{0}\right), \theta_{0}\right)\right)
$$

for $\theta \in\left[\theta_{w}, \theta_{0}\right]$, and

$$
c\left(\rho_{0}\right) \leq \bar{c}\left(\rho^{\varepsilon, \delta}\left(r^{\varepsilon, \delta}\left(\theta_{0}\right), \theta_{0}\right), \rho_{0}\right) \leq r^{\varepsilon, \delta}\left(\theta_{0}\right) .
$$

Thus,

$$
\rho^{\varepsilon, \delta}\left(r^{\varepsilon, \delta}\left(\theta_{0}\right), \theta_{0}\right) \rightarrow \rho_{0} .
$$

Therefore, $r(\theta) \equiv c\left(\rho_{0}\right)$ for $\theta \in\left[\theta_{w}, \theta_{0}\right]$. 
Next we prove the continuity of solutions up to the boundary where $r(\theta)=c\left(\rho_{0}\right)$. First, we prove that $r \in C^{1}$. Still from Lemma 3.6, we obtain that

$$
\rho(r(\theta), \theta) \rightarrow \rho_{0} \quad \text { if } \theta \rightarrow \theta_{0} \text { from the right. }
$$

On the other hand,

$$
r^{\prime}(\theta)=r(\theta) \sqrt{\frac{r^{2}(\theta)-\bar{c}^{2}}{\bar{c}^{2}}} \quad \text { for } \theta>\theta_{0},
$$

which implies that $r^{\prime}(\theta) \rightarrow 0$ as $\theta \rightarrow \theta_{0}$ from the right-hand side, and it holds obviously from the left-hand side. If we define $r^{\prime}\left(\theta_{0}\right)=0$, then $r \in C^{1}$.

Note that the equation for $u=c^{2}(\rho)$ is

$$
\begin{aligned}
Q(u):= & \left(c^{2}-r^{2}+\varepsilon\right) u_{r r}+\frac{c^{2}+\varepsilon}{r^{2}} u_{\theta \theta}+\frac{c^{2}+(\gamma-2)\left(r^{2}-\varepsilon\right)}{(\gamma-1) c^{2}}\left(u_{r}\right)^{2} \\
& +\frac{1}{(\gamma-1) r^{2}}\left(u_{\theta}\right)^{2}+\frac{c^{2}-2 r^{2}+\varepsilon}{r} u_{r} \\
= & 0 .
\end{aligned}
$$

We prove the most complicated case $\theta_{0}=\theta_{a}$ first, and the other cases will be discussed later.

We construct a family of barrier functions $\left\{\Psi_{\tau}\right\}$ with parameter $\tau$. For any $m>0$, there exists $\delta_{1}(m)>0$ such that $r^{\prime}(\theta)<m$ for $\left|\theta-\theta_{a}\right|<\delta_{1}(m)$. This implies that

$$
\left|r(\theta)-r\left(\theta_{a}\right)\right|<m \delta_{1}(m) \quad \text { for }\left|\theta-\theta_{a}\right|<\delta_{1}(m),
$$

where $\delta_{1}(m) \rightarrow 0$ as $m \rightarrow 0$.

Let $m<1$ and $m \delta(m)=\frac{\tau}{2}$ ( $\tau$ will be specified later). We have

$$
\begin{aligned}
\rho_{0} \leq \rho(r(\theta), \theta) & \leq \rho\left(r\left(\theta_{a}+\delta_{1}(m)\right), \theta_{a}+\delta_{1}(m)\right) \leq\left(\bar{c}_{\rho_{0}}\right)^{-1}\left(r\left(\theta_{a}+\delta_{1}(m)\right)\right) \\
& \leq \rho_{0}+\frac{C m}{2} .
\end{aligned}
$$

For $\varepsilon, \delta$ small enough, we obtain

$$
\rho_{0} \leq \rho^{\varepsilon, \delta}\left(r^{\varepsilon, \delta}(\theta), \theta\right) \leq \rho_{0}+C m
$$

and

$$
0 \leq r^{\varepsilon, \delta}(\theta)-c\left(\rho_{0}\right)<\tau \quad \text { for }\left|\theta-\theta_{a}\right|<\delta_{1}(m),
$$

where $C$ depends only on $\gamma$ and $\rho_{0}$. We define

$$
\Psi_{\tau}=\Psi_{\tau}^{\varepsilon, \delta}=c^{2}\left(\rho_{0}+C m\right)+A\left(c\left(\rho_{0}\right)+\tau-r\right)^{\alpha}+B\left(\theta-\theta_{a}\right)^{2}
$$

in

$$
Q^{\varepsilon, \delta}=\left\{(r, \theta):\left|r-c\left(\rho_{0}\right)\right| \leq \delta_{2},\left|\theta-\theta_{a}\right| \leq \delta_{1}(m)\right\} \cap \Omega^{\varepsilon, \delta},
$$

where $\delta_{2}>\tau$ will be chosen later.

Choose

$$
B=\frac{c^{2}\left(\rho_{1}\right)-c^{2}\left(\rho_{0}\right)}{\delta_{1}^{2}(m)}, \quad A=A_{1}=\frac{c^{2}\left(\rho_{1}\right)-c^{2}\left(\rho_{0}\right)}{\delta_{2}^{\alpha}} .
$$


Since $\rho_{0} \leq \rho^{\varepsilon, \delta} \leq \rho_{1}$ and $\rho^{\varepsilon, \delta} \leq \rho_{0}+C m$ on $\Gamma_{\text {shock }}^{\varepsilon, \delta} \cap \partial Q^{\varepsilon, \delta}$, we have

$$
\Psi_{\tau} \geq c^{2}\left(\rho^{\varepsilon, \delta}\right) \quad \text { on } \partial Q^{\varepsilon, \delta} \text {. }
$$

Thus, we have

$$
\begin{aligned}
Q\left(\Psi_{\tau}^{\varepsilon, \delta}\right)= & A \alpha(\alpha-1)\left(c^{2}\left(\rho^{\varepsilon, \delta}\right)-r^{2}\right)\left(c\left(\rho_{0}\right)-r+\tau\right)^{\alpha-2}+\frac{2 B c^{2}}{r^{2}} \\
& +\left(1-\frac{\gamma-2}{(\gamma-1) c^{2}}\left(c^{2}-r^{2}\right)\right) A^{2} \alpha^{2}\left(c\left(\rho_{0}\right)-r+\tau\right)^{2 \alpha-2} \\
& +\frac{4 B^{2}}{(\gamma-1) r^{2}}\left(\theta-\theta_{a}\right)^{2}-\frac{A \alpha\left(c^{2}-2 r^{2}\right)}{r}\left(c\left(\rho_{0}\right)-r+\tau\right)^{\alpha-1} .
\end{aligned}
$$

Consider (4.2) in $Q^{\varepsilon, \delta} \cap\left\{(r, \theta): c^{2}\left(\rho^{\varepsilon, \delta}\right)-\Psi_{\tau}^{\varepsilon, \delta} \geq 0\right\}$. Since

$$
c\left(\rho_{0}+C m\right) \geq c\left(\rho^{\varepsilon, \delta}\left(r^{\varepsilon, \delta}(\theta), \theta\right)\right) \geq r^{\varepsilon, \delta}(\theta) \geq r,
$$

we have

$$
\begin{aligned}
c^{2}\left(\rho^{\varepsilon, \delta}\right)-r^{2}+\varepsilon & \geq c^{2}\left(\rho^{\varepsilon, \delta}\right)-\Psi_{\tau}^{\varepsilon, \delta}+c^{2}\left(\rho_{0}+C m\right)-r^{2}+A\left(c\left(\rho_{0}\right)+\tau-r\right)^{\alpha} \\
& \geq A\left(c\left(\rho_{0}\right)+\tau-r\right)^{\alpha} .
\end{aligned}
$$

For $\alpha<1$, (4.2) implies

$$
\begin{aligned}
Q\left(\Psi_{\tau}^{\varepsilon, \delta}\right) \leq & A^{2} \alpha\left(\left(2-\frac{\gamma-2}{(\gamma-1) c^{2}}\left(c^{2}-r^{2}\right)\right) \alpha-1\right)\left(c\left(\rho_{1}\right)-r+\tau\right)^{2 \alpha-2} \\
& -\frac{c^{2}-2 r^{2}}{r} A \alpha\left(c\left(\rho_{1}\right)-r+\tau\right)^{\alpha-1}+\frac{4}{(\gamma-1) r^{2}} B^{2}\left(\theta-\theta_{a}\right)^{2}+2 B \frac{c^{2}}{r^{2}} .
\end{aligned}
$$

Moreover, let

$$
A>A_{1}, \quad B=\frac{C\left(\rho_{0}, \rho_{1}\right)}{\delta_{1}^{2}(m)} .
$$

If $\alpha<\frac{1}{2+C\left(\rho_{0}, \rho_{1}, \gamma\right)}$ and $\delta_{2}+\tau$ is small enough, we have

$$
\begin{aligned}
Q\left(\Psi_{\tau}^{\varepsilon, \delta}\right) \leq & C\left(\rho_{0}, \rho_{1}\right)\left(\left(2+C\left(\rho_{0}, \rho_{1}, \gamma\right)\right) \alpha-1\right) A^{2}\left(c\left(\rho_{1}\right)+\tau-r\right)^{2 \alpha-2} \\
& +\frac{C\left(\rho_{1}, \rho_{2}\right)}{\delta_{1}^{2}(m)} .
\end{aligned}
$$

Then there exists a constant $A_{2}\left(\delta_{2}, m, \rho_{0}, \rho_{1}\right)$ such that

$$
Q\left(\Psi_{\tau}^{\varepsilon, \delta}\right) \leq 0 \quad \text { for } A>A_{2} .
$$

In fact, if $r<c\left(\rho_{0}\right)$, we choose $\delta_{2}=\sqrt{m} \delta_{1}(m)$ to obtain

$$
c\left(\rho_{0}\right)-r+\tau \leq 2 \sqrt{m} \delta_{1}(m),
$$

and let

$$
A_{2}^{(1)}=\frac{C\left(\rho_{0}, \rho_{1}, \alpha\right) m^{\frac{1-\alpha}{2}}}{\delta_{1}^{\alpha}(m)}, \quad A_{1}=\frac{C\left(\rho_{0}, \rho_{1}\right)}{m^{\frac{\alpha}{2}} \delta_{1}^{\alpha}(m)} .
$$

If $r \geq c\left(\rho_{0}\right)$

$$
c\left(\rho_{0}\right)+\tau-r \leq \tau,
$$

and we let

$$
A_{2}^{(2)}=\frac{C\left(\rho_{0}, \rho_{1}, \alpha\right) m^{1-\alpha}}{\delta_{1}^{\alpha}(m)} .
$$


Set $A=\max \left\{A_{1}, A_{2}^{(1)}, A_{2}^{(2)}\right\}$. Then $\rho^{\varepsilon, \delta} \leq \Psi_{\tau}^{\varepsilon, \delta}$ in $Q^{\varepsilon, \delta}$. Passing to the limits $\delta, \varepsilon \rightarrow 0$, we obtain

$$
\rho \leq \Psi_{\tau} \quad \text { in the domain } Q\left(m, \sqrt{m} \delta_{1}(m)\right):=\cap_{\delta, \varepsilon>0} Q^{\varepsilon, \delta} .
$$

With these barrier functions, we can show that $\rho$ is continuous at $\left(r\left(\theta_{a}\right), \theta_{a}\right)$. In fact, for every $\varepsilon_{1}>0$, there exists $m>0$ such that

$$
c^{2}\left(\rho_{0}+C m\right)-c^{2}\left(\rho_{0}\right)<\frac{\varepsilon_{1}}{3} .
$$

For this $m$, we can choose $A, B$, and $\tau$ such that

$$
c^{2}(\rho) \leq \Psi_{\tau} \leq \frac{\varepsilon_{1}}{3}+c^{2}\left(\rho_{1}\right)+A\left(c\left(\rho_{0}\right)-r+\tau\right)^{\alpha}+B\left(\theta-\theta_{a}\right)^{2} .
$$

Choose the neighborhood of $\left(r\left(\theta_{a}\right), \theta_{a}\right)$ small enough so that

$$
A\left(c\left(\rho_{0}\right)-r+\tau\right)^{\alpha} \leq A(2 \tau)^{\alpha} \leq C\left(\rho_{0}, \rho_{1}, \alpha\right) m^{\frac{\alpha}{2}} .
$$

Then, choosing $m$ small again, we have

$$
c^{2}(\rho) \leq \frac{2 \varepsilon_{1}}{3}+c^{2}\left(\rho_{0}\right)+B\left(\theta-\theta_{a}\right)^{2} .
$$

Finally, we choose a small neighborhood such that

$$
c^{2}\left(\rho_{0}\right) \leq c^{2}(\rho) \leq \varepsilon_{1}+c^{2}\left(\rho_{0}\right) .
$$

Thus, we obtain our claim that $\rho$ is continuous at $\left(r\left(\theta_{a}\right), \theta_{a}\right)$, that is, the results hold for this case.

As for the case $\theta \in\left[\theta_{w}, \theta_{a}\right)$, we can choose arbitrary $\tau>0$, which is independent of the neighborhood of $\theta$. This fact makes the similar proof of this case much easier for all sufficiently small $\varepsilon$ and $\delta$, and we omit the details here.

Next, we discuss the wave strength at the sonic circle $r \equiv c\left(\rho_{0}\right)$ and conclude that Case 3 in Lemma 4.2 does not actually occur.

Lemma 4.3. Let $r(\theta)$ be monotone increasing in $\theta$ on $\Gamma_{\text {shock }}$ and $\rho>\rho_{0}$ in the subsonic region. Then $r(\theta)>c\left(\rho_{0}\right)$ for $\theta_{w}<\theta \leq \theta_{1}$.

Proof. We divide the proof into five steps.

1. We show our claim by contradiction. More precisely, if there exists $\bar{\theta}$ such that $r(\bar{\theta})=c\left(\rho_{0}\right):=c_{0}$. Then, using the monotonicity of $r(\theta)$,

$$
r(\theta) \equiv c_{0} \quad \text { for } \theta_{w} \leq \theta \leq \bar{\theta} .
$$

2. For $\theta_{0} \in\left[\theta_{w}, \bar{\theta}\right]$, we define

$$
w_{1}=c_{0}^{2}+A_{1}\left(c_{0}-r\right)^{\frac{1}{2}}-B_{1}\left(c_{0}-r\right)^{\beta_{1}}+D_{1}\left(\theta-\theta_{0}\right)^{2},
$$

where $A_{1}, B_{1}, D_{1}>0$ and $\frac{1}{2}<\beta_{1}<1$, which will be specified later to prove that $\rho \in C^{\frac{1}{2}}$ near this boundary point. 
Using (4.1) with the coefficient of $u_{r r}$ replaced by $u-r^{2}$, we have

$$
\begin{aligned}
\hat{Q}\left(w_{1}\right)= & \left(-\left(\beta_{1}^{2}-\frac{1}{4}\right) A_{1} B_{1}\left(c_{0}-r\right)^{\beta_{1}-\frac{3}{2}}+O_{1}\right) \\
& +\left(\beta_{1}\left(2 \beta_{1}-1\right) B_{1}^{2}\left(c_{0}-r\right)^{2 \beta_{1}-2}+O_{2}\right) \\
& -\frac{(\gamma-2)}{4(\gamma-1) c^{2}} A_{1}^{2}\left(c^{2}-r^{2}\right)\left(c_{0}-r\right)^{-1} \\
& +\left(-\frac{A_{1} D_{1}}{4}\left(c_{0}-r\right)^{-\frac{3}{2}}\left(\theta-\theta_{0}\right)^{2}+O_{4}\right),
\end{aligned}
$$

where

$$
\begin{aligned}
O_{1}= & -\frac{A_{1}}{2} c_{0}\left(c_{0}-r\right)^{-\frac{1}{2}}-2 c_{0} \beta_{1}\left(\beta_{1}-1\right) B_{1}\left(c_{0}-r\right)^{\beta_{1}-1}+\frac{A_{1}}{4}\left(c_{0}-r\right)^{\frac{1}{2}} \\
& +\beta_{1}\left(\beta_{1}-1\right) B_{1}\left(c_{0}-r\right)^{\beta_{1}}+\frac{2 c^{2}}{r^{2}} D_{1}+\frac{A_{1} r}{2}\left(c_{0}-r\right)^{-\frac{1}{2}}-\beta_{1} B_{1} r\left(c_{0}-r\right)^{\beta_{1}-1} \\
& +\frac{(\gamma-2) \beta_{1} A_{1} B_{1}}{(\gamma-1) c^{2}}\left(c^{2}-r^{2}\right)\left(c_{0}-r\right)^{\beta_{1}-\frac{3}{2}}-\frac{A_{1}}{2 r}\left(c^{2}-r^{2}\right)\left(c_{0}-r\right)^{\frac{1}{2}} \\
& +\frac{\beta_{1} B_{1}}{r}\left(c^{2}-r^{2}\right)\left(c_{0}-r\right)^{\beta_{1}-1}, \\
O_{2}= & -\frac{(\gamma-2) \beta_{1}^{2} B_{1}^{2}}{(\gamma-1) c^{2}}\left(c^{2}-r^{2}\right)\left(c_{0}-r\right)^{2 \beta_{1}-2}, \\
O_{4}= & \beta_{1}\left(\beta_{1}-1\right) B_{1} D_{1}\left(c_{0}-r\right)^{\beta_{1}-2}\left(\theta-\theta_{0}\right)^{2}+\frac{4 D_{1}}{(\gamma-1) r^{2}}\left(\theta-\theta_{0}\right)^{2} .
\end{aligned}
$$

Notice that there exists $0<\alpha<\frac{1}{2}$ such that $c^{2}-r^{2} \leq\left(c_{0}-r\right)^{\alpha}$ for $c_{0}-r>0$ small. Thus,

$$
\left|\frac{(\gamma-2)}{4(\gamma-1) c^{2}} A_{1}^{2}\left(c^{2}-r^{2}\right)\left(c_{0}-r\right)^{-1}\right| \leq C\left(\rho_{0}, \rho_{1}\right) A_{1}^{2}\left(c_{0}-r\right)^{\alpha-1} .
$$

We can choose a proper constant $\alpha$ such that $\beta_{1}-\frac{3}{2}<\alpha-1$, i.e., $\alpha>\beta_{1}-\frac{1}{2}$.

On one hand, let $c_{0}-r>0$ be small enough so that

$$
\left(\beta_{1}^{2}-\frac{1}{4}\right) A_{1} B_{1}\left(c_{0}-r\right)^{\beta_{1}-\frac{3}{2}}>3 C\left(\rho_{0}, \rho_{1}\right) A_{1}^{2}\left(c_{0}-r\right)^{\alpha-1},
$$

which implies

$$
B_{1}>\frac{3 C\left(\rho_{0}, \rho_{1}\right)}{\beta_{1}^{2}-\frac{1}{4}} A_{1}\left(c_{0}-r\right)^{\alpha-\beta_{1}+\frac{1}{2}}:=A_{1} C\left(\rho_{0}, \rho_{1}, \beta_{1}\right)\left(c_{0}-r\right)^{\alpha-\beta_{1}+\frac{1}{2}} .
$$

On the other hand, if $c_{0}-r>0$ is sufficiently small, we have

$$
\left(\beta_{1}^{2}-\frac{1}{4}\right) A_{1} B_{1}\left(c_{0}-r\right)^{\beta_{1}-\frac{3}{2}}>3 \beta_{1}\left(2 \beta_{1}-1\right) B_{1}^{2}\left(c_{0}-r\right)^{2 \beta_{1}-2},
$$

which implies

$$
A>\frac{\left(2 \beta_{1}^{2}-\beta_{1}\right) B_{1}}{\beta_{1}^{2}-\frac{1}{4}}\left(c_{0}-r\right)^{\beta_{1}-\frac{1}{2}}:=C\left(\beta_{1}\right) B_{1}\left(c_{0}-r\right)^{\beta_{1}-\frac{1}{2}} .
$$

Moreover, we have

$$
C\left(\rho_{0}, \rho_{1}, \beta_{1}\right)\left(c_{0}-r\right)^{\alpha-\beta_{1}+\frac{1}{2}}<C\left(\beta_{1}\right)\left(c_{0}-r\right)^{\frac{1}{2}-\beta_{1}}
$$

when $r \in\left[\bar{r}, c_{0}\right]$, and $\bar{r}$ is close to $c_{0}$. 
Choose proper constants $A_{1}$ and $D_{1}$ such that

$$
w_{1}>c_{0}^{2}+\frac{1}{2} A_{1}\left(c_{0}-r\right)^{\frac{1}{2}}+D_{1}\left(\theta-\theta_{0}\right)^{2}>c^{2}
$$

at the boundary of a relatively neighborhood $N_{1}$ of $\left(c_{0}, \theta_{0}\right)$ to $\Omega$. Choose $B_{1}$ sufficiently small such that

$$
\hat{Q}\left(w_{1}\right)<0
$$

and

$C\left(\rho_{0}, \rho_{1}, \beta_{1}\right)\left(c_{0}-r\right)^{\alpha-\beta+\frac{1}{2}}<\frac{B_{1}}{A_{1}}<\min \left\{C(\beta), \frac{1}{4\left(\beta_{1}-\beta_{1}^{2}\right)}\right\}\left(c_{0}-r\right)^{\frac{1}{2}-\beta_{1}} \quad$ in $N_{1}$.

This implies that (4.4) and (4.5) hold.

Obviously, we have

$$
\partial_{r r} w_{1}<0 \quad \text { in } N_{1}
$$

if (4.4) and (4.5) hold. If $S_{1}=\left\{(r, \theta) \in N_{1}: c^{2}>w_{1}\right\} \neq \emptyset$, we have $Q\left(w_{1}\right) \leq$ $\hat{Q}\left(w_{1}\right)<0$ in $S_{1}$. Thus,

$$
0<Q u-Q\left(w_{1}\right)
$$

Using the maximum principle, $u \leq w_{1}$, which contradicts with $c^{2}>w_{1}$. Thus

$$
c^{2} \leq w_{1} \quad \text { in } N_{1}
$$

3. We define

$$
w_{2}=c_{0}^{2}+A_{2}\left(c_{0}-r\right)^{\frac{1}{2}}+B_{2}\left(c_{0}-r\right)^{\beta_{2}}-D_{2}\left(\theta-\theta_{0}\right)^{2},
$$

where $A_{2}, B_{2}, D_{2}>0$ and $\frac{1}{2}<\beta_{2}<1$, all of which will be specified later to prove that $C^{\frac{1}{2}}$ is optimal. Through a simple algebraic calculation, we have

$$
\begin{aligned}
\hat{Q}\left(w_{2}\right)= & \left(\left(\beta_{2}^{2}-\frac{1}{4}\right) A_{2} B_{2}\left(c_{0}-r\right)^{\beta_{2}-\frac{3}{2}}+\bar{O}_{1}\right)+\left(\beta_{2}\left(2 \beta_{2}-1\right) B_{2}^{2}\left(c_{0}-r\right)^{2 \beta_{2}-2}+\bar{O}_{2}\right) \\
& +\left(-\beta_{2}\left(\beta_{2}-1\right) B_{2} D_{2}\left(c_{0}-r\right)^{\beta_{2}-2}\left(\theta-\theta_{0}\right)^{2}+\bar{O}_{3}\right) \\
& +\left(\beta_{2} B_{2} r\left(c_{0}-r\right)^{\beta_{2}-1}+\bar{O}_{4}\right)+\frac{1}{4} A_{2} D_{2}\left(c_{0}-r\right)^{-\frac{3}{2}}\left(\theta-\theta_{0}\right)^{2} \\
& +2 \beta_{2}\left(\beta_{2}-1\right) B_{2} c_{0}\left(c_{0}-r\right)^{\beta_{2}-1},
\end{aligned}
$$


where

$$
\begin{aligned}
\bar{O}_{1}= & -\frac{(\gamma-2)}{4(\gamma-1) c^{2}} A_{2}^{2}\left(c^{2}-r^{2}\right)\left(c_{0}-r\right)^{-1}-\frac{A_{2}}{2} c_{0}\left(c_{0}-r\right)^{-\frac{1}{2}}+\frac{A_{2}}{4}\left(c_{0}-r\right)^{\frac{1}{2}} \\
& -\beta_{2}\left(\beta_{2}-1\right) B_{2}\left(c_{0}-r\right)^{\beta_{2}}-\frac{2 c^{2}}{r^{2}} D_{2}+\frac{A_{2} r}{2}\left(c_{0}-r\right)^{-\frac{1}{2}}-\frac{A_{2}}{2 r}\left(c^{2}-r^{2}\right)\left(c_{0}-r\right)^{\frac{1}{2}} \\
& -\frac{(\gamma-2) \beta_{2} A_{2} B_{2}}{(\gamma-1) c^{2}}\left(c^{2}-r^{2}\right)\left(c_{0}-r\right)^{\beta_{2}-\frac{3}{2}}, \\
\bar{O}_{2}= & -\frac{(\gamma-2) \beta_{2}^{2} B_{2}^{2}}{(\gamma-1) c^{2}}\left(c^{2}-r^{2}\right)\left(c_{0}-r\right)^{2 \beta_{2}-2}, \\
\bar{O}_{3}= & -\frac{4 D_{2}}{(\gamma-1) r^{2}}\left(\theta-\theta_{0}\right)^{2}, \\
\bar{O}_{4}= & -\frac{\beta_{2} B_{2}}{r}\left(c^{2}-r^{2}\right)\left(c_{0}-r\right)^{\beta_{2}-1} .
\end{aligned}
$$

Let $D_{2}$ be large enough such that $c^{2}>w_{2}$ for some $\theta=\theta_{a}, \theta_{b}$. We choose $\tilde{r}<c_{0}$ such that

$$
\begin{aligned}
c^{2} & >c_{0}^{2}+2 A_{2}\left(c_{0}-\tilde{r}\right)^{\frac{1}{2}}-D_{2}\left(\theta-\theta_{0}\right)^{2} \\
& \geq c_{0}+A_{2}\left(c_{0}-\tilde{r}\right)^{\frac{1}{2}}+B_{2}\left(c_{0}-\tilde{r}\right)^{\beta_{2}}-D_{2}\left(\theta-\theta_{0}\right)^{2} .
\end{aligned}
$$

The second inequality holds, provided that $\frac{B_{2}}{A_{2}} \leq\left(c_{0}-\tilde{r}\right)^{\frac{1}{2}-\beta_{2}}$. Choosing $\beta_{2}>\frac{7}{8}$, we have

$$
\frac{1}{2} \beta_{2} B_{2} r\left(c_{0}-r\right)^{\beta_{2}-1}+2 \beta_{2}\left(\beta_{2}-1\right) B_{2} c_{0}\left(c_{0}-r\right)^{\beta_{2}-1} \leq 0 \quad \text { for } \frac{c_{0}}{2}<r<c_{0},
$$

and

$$
\hat{Q}\left(w_{2}\right)>0 \text {. }
$$

Then, if $S_{2}=\left\{(r, \theta) \in N_{1}: c^{2}<w_{2}\right\} \neq \emptyset$, we have

$$
Q\left(w_{2}\right) \geq \hat{Q}\left(w_{2}\right)>0 \quad \text { in } S_{2} .
$$

Thus, $Q u-Q\left(w_{2}\right)<0$. Using the maximum principle, $c \leq w_{2}$, which contradicts with $c^{2}<w_{1}$. Thus

$$
c^{2} \geq w_{2} \quad \text { in } N_{2} \text {. }
$$

4. We now show that

$$
c^{2}>c_{0}^{2}+A_{3}\left(c_{0}-r\right)^{\frac{1}{2}}+B_{3}\left(c_{0}-r\right)^{\beta_{3}}=: w_{3}
$$

in a relative neighborhood of $\left(r_{0}, \theta_{0}\right)$, where $A_{3}$ and $B_{3}$ are positive constants to be specified later, so that the $C^{\frac{1}{2}}$-regularity is optimal.

Since $c^{2} \geq w_{2}$, we can choose $\bar{\theta}_{a}$ and $\bar{\theta}_{b}$ such that

$$
c^{2} \geq c_{0}^{2}+A_{2}\left(\bar{\theta}_{a}, \bar{\theta}_{a}\right)\left(c_{0}-r\right)^{\frac{1}{2}}+B_{2}\left(c_{0}-r\right)^{\beta_{2}} \quad \text { for } N_{3} \subset N_{2} .
$$

Thus, there exist positive constants $A_{3}, B_{3}$, and $\beta_{3}$ such that

$$
w_{3} \leq c^{2} \text {. }
$$


It is easy to see that

$$
\begin{aligned}
\hat{Q}\left(w_{3}\right)= & \left(\left(\beta_{3}^{2}-\frac{1}{4}\right) A_{3} B_{3}\left(c_{0}-r\right)^{\beta_{3}-\frac{3}{2}}+\tilde{O}_{1}\right)+\left(\beta_{3}\left(2 \beta_{3}-1\right) B_{3}^{2}\left(c_{0}-r\right)^{2 \beta_{3}-2}+\tilde{O}_{2}\right) \\
& +\left(\beta_{3} B_{3} r\left(c_{0}-r\right)^{\beta_{3}-1}+\tilde{O}_{4}\right)+2 \beta_{3}\left(\beta_{3}-1\right) B_{3} c_{0}\left(c_{0}-r\right)^{\beta_{3}-1},
\end{aligned}
$$

where

$$
\begin{aligned}
\tilde{O}_{1}= & -\frac{(\gamma-2)}{4(\gamma-1) c^{2}} A_{3}^{2}\left(c^{2}-r^{2}\right)\left(c_{0}-r\right)^{-1}-\frac{A_{3}}{2} c_{0}\left(c_{0}-r\right)^{-\frac{1}{2}}+\frac{A_{3}}{4}\left(c_{0}-r\right)^{\frac{1}{2}} \\
& +\frac{A_{3} r}{2}\left(c_{0}-r\right)^{-\frac{1}{2}}-\frac{A_{3}}{2 r}\left(c^{2}-r^{2}\right)\left(c_{0}-r\right)^{\frac{1}{2}} \\
& -\frac{(\gamma-2) \beta_{3} A_{3} B_{3}}{(\gamma-1) c^{2}}\left(c^{2}-r^{2}\right)\left(c_{0}-r\right)^{\beta_{3}-\frac{3}{2}}, \\
\tilde{O}_{2}= & \beta_{3}\left(\beta_{3}-1\right) B_{3}\left(c_{0}-r\right)^{\beta_{3}}-\frac{(\gamma-2) \beta_{3}^{2} B_{3}^{2}}{(\gamma-1) c^{2}}\left(c^{2}-r^{2}\right)\left(c_{0}-r\right)^{2 \beta_{3}-2}, \\
\tilde{O}_{3}= & -\frac{\beta_{3} B_{3}}{r}\left(c^{2}-r^{2}\right)\left(c_{0}-r\right)^{\beta_{3}-1} .
\end{aligned}
$$

Similarly, we can show that $c^{2} \geq w_{3}$ in $N_{3}$.

Thus, $\frac{1}{2} A_{3}\left(c_{0}-r\right)^{\frac{1}{2}} \leq c^{2}-c_{0}^{2} \leq 2 A_{1}\left(c_{0}-r\right)^{\frac{1}{2}}$ in $N_{1} \cap N_{3}$. This implies

$$
a\left(c_{0}-r\right)^{\frac{1}{2}} \leq v:=\rho-\rho_{0} \leq A\left(c_{0}-r\right)^{\frac{1}{2}} \quad \text { in } N_{1} \cap N_{3}
$$

for some constants $a$ and $A$, so the optimal regularity of $\rho$ is $C^{\frac{1}{2}}$ near the sonic circle.

5. We introduce the coordinates: $(x, y)=\left(c_{0}-r, \theta-\theta_{w}\right)$ and set $v=c^{2}-c_{0}^{2}$. Thus, rewriting the equation for $c^{2}$ in the divergence form, we have

$$
Q v=\left(a_{11}\left(v+2 c_{0} x-x^{2}\right) v_{x}\right)_{x}+b_{1} v_{x}+\left(a_{22} v_{y}\right)_{y}=0
$$

where $a_{11}=\frac{c^{\frac{2(2-\gamma)}{\gamma-1}}}{\gamma-1}, a_{22}=\frac{c^{\frac{2}{\gamma-1}}}{(\gamma-1) r^{2}}$, and $b_{1}=\frac{c^{\frac{2}{\gamma-1}}}{(\gamma-1) r}$.

Scale $v$ in $N_{1} \cap N_{3}$ by defining

$$
u(S, T)=\frac{1}{S^{\frac{1}{5}}} v\left(S^{-\frac{12}{5}}, y_{0}+S^{-\frac{14}{5}} T\right)
$$

for $\left(S^{-\frac{12}{5}}, y_{0}+S^{-\frac{14}{5}} T\right) \in N_{1} \cap N_{3}$. Then $u$ satisfies the following governing equation: (4.9)

$Q u=\left(\tilde{a}_{11} u_{S}\right)_{S}+\left(\tilde{a}_{12} u_{T}\right)_{S}+\left(\tilde{a}_{21} u_{S}\right)_{T}+\left(\tilde{a}_{22} u_{T}\right)_{T}+\left(\tilde{b}_{2} u\right)_{T}+\tilde{c}_{1} u_{S}+\tilde{c}_{2} u_{T}+\tilde{d}_{2} u=0$, 
where

$$
\begin{aligned}
\tilde{a}_{11}= & a_{11}\left(S^{\frac{7}{5}} u+2 c_{0} S^{-\frac{6}{5}}-S^{-\frac{18}{5}}\right), \\
\tilde{a}_{12}= & \frac{14 T}{5 S} a_{11}\left(S^{\frac{7}{5}} u+2 c_{0} S^{-\frac{6}{5}}-S^{-\frac{18}{5}}\right), \\
\tilde{a}_{21}= & \frac{14 T}{5 S} a_{11}\left(S^{\frac{7}{5}} u+2 c_{0} S^{-\frac{6}{5}}-S^{-\frac{18}{5}}\right), \\
\tilde{a}_{22}= & \frac{144}{25} a_{22}+\frac{189 T^{2}}{25 S^{2}} a_{11}\left(S^{\frac{7}{5}} u+2 c_{0} S^{-\frac{6}{5}}-S^{-\frac{18}{5}}\right), \\
\tilde{b}_{2}= & \frac{14 T}{25 S^{2}} a_{11}\left(S^{\frac{7}{5}} u+2 c_{0} S^{-\frac{6}{5}}-S^{-\frac{18}{5}}\right)=\tilde{b}_{22} T S^{-2}, \\
\tilde{c}_{1}= & \frac{S^{\frac{7}{5}} u(2-\gamma) S^{-\frac{11}{5}}}{5(\gamma-1)^{2}} c^{\frac{2(3-2 \gamma)}{\gamma-1}}\left(S^{\frac{7}{5}} u+2 c_{0} S^{-\frac{6}{5}}-S^{-\frac{18}{5}}\right) \\
& -\frac{a_{11}\left(4 c_{0}-S^{-\frac{12}{5}}\right)}{5 S^{\frac{11}{5}}}-\frac{12 b_{1}}{5 S^{\frac{11}{5}}}=\tilde{c}_{11} S^{-\frac{11}{5}}, \\
\tilde{c}_{2}= & \frac{168 T}{25 S^{2}} a_{11}\left(S^{\frac{7}{5}} u+2 c_{0} S^{-\frac{6}{5}}-S^{-\frac{18}{5}}\right)-\frac{189 T a_{11}}{25 S^{2}}-\frac{168 b_{1} T}{25 S^{\frac{16}{5}}}=\tilde{c}_{22} S^{-2} T, \\
\tilde{d}= & \frac{S^{\frac{7}{5}} u(2-\gamma) S^{-\frac{16}{5}}}{25(\gamma-1)^{2}} c^{\frac{2(3-2 \gamma)}{\gamma-1}}\left(S^{\frac{7}{5}} u+2 c_{0} S^{-\frac{6}{5}}-S^{-\frac{18}{5}}\right) \\
& -\frac{13 a_{11}\left(2 c_{0}-S^{-\frac{12}{5}}\right)}{25 S^{\frac{16}{5}}}-\frac{12 a_{11} S^{\frac{7}{5}} u\left(c_{0}+\frac{6}{5} S^{-\frac{6}{5}}\right)}{25 S^{\frac{23}{5}}}-\frac{12 b_{1}}{25 S^{\frac{16}{5}}}=\tilde{d}_{1} S^{-\frac{16}{5}} .
\end{aligned}
$$

From the optimal continuity,

$$
0<a \leq S^{\frac{7}{5}} u \leq A
$$

we have

$$
0<C^{-1} \leq \lambda_{1}, \lambda_{2}, \tilde{b}_{22}, \tilde{c}_{11}, \tilde{c}_{22}, \tilde{d}_{1} \leq C
$$

if $S^{-1}$ and $T$ are sufficiently small. Here $\lambda_{1}$ and $\lambda_{2}$ are the eigenvalues of the matrix $\left(\tilde{a}_{i j}\right)_{2 \times 2}$, so the equation is uniformly elliptic for $u$ in the $(S, T)$-coordinates.

Let $x_{0}^{-1}<S \leq x_{0}^{-\frac{5}{4}}$ with $x_{0}$ small enough. Then, using Theorem 8.20 in [11], we have

$$
\begin{aligned}
a x_{0}^{\frac{7}{5}} \leq u\left(x_{0}^{-1}, 0\right) & \leq \sup _{x_{0}^{-1} \leq S \leq x_{0}^{-5 / 4}} u(S, T) \\
& \leq C \inf _{x_{0}^{-1} \leq S \leq x_{0}^{-5 / 4}} u(S, T) \leq C u\left(x_{0}^{-\frac{5}{4}}, 0\right) \leq C A x_{0}^{\frac{7}{4}},
\end{aligned}
$$

where $C \leq C(n)^{\left(\frac{\Lambda}{\lambda}+\nu R\right)}$ in [1] is independent of $x_{0}$, since $(\Lambda, \lambda)=\left(\lambda_{1}, \lambda_{2}\right), R=$ $x_{0}^{-\frac{5}{4}}-x_{0}^{-1} \leq x_{0}^{-\frac{5}{4}}$, and $\nu:=\max _{x_{0}^{-1} \leq S \leq x_{0}^{-5 / 4}}\left\{\tilde{b}_{2}, \tilde{c}_{1}, \tilde{c}_{2}, \sqrt{\tilde{d}}\right\} \leq C x_{0}^{\frac{8}{5}}$. This implies that 
$x_{0}^{-\frac{7}{20}} \leq C$, which is a contradiction if $x_{0}$ is sufficiently small. This completes the proof.

Next, we consider $\Gamma_{\text {shock }}$ in the $(\xi, \eta)$-coordinates to obtain finer properties.

Lemma 4.4. For the free boundary $\Gamma_{\text {shock }}=\left\{(\xi, \eta(\xi)): \xi_{w}<\xi<\xi_{1}\right\}$ determined by (3.2) - (3.7),

$$
\eta(\xi) \in C^{2}\left(\left[\xi_{w}, \xi_{1}\right)\right)
$$

moreover, $\eta(\xi)$ is strictly convex for $\xi \in\left[\xi_{w}, \xi_{1}\right)$.

Proof. We define

$$
F(\xi, \eta)=\xi^{2}+\eta^{2}-r^{2}(\theta(\xi, \eta))=0 \quad \text { on } \Gamma_{\text {shock }} .
$$

It is easy to check that

$$
F_{\eta}=\left.\left(2 \eta-2 r r^{\prime} \theta_{\eta}\right)\right|_{\xi=\xi_{w}}=2 \eta\left(\xi_{w}\right) \neq 0 .
$$

By the implicit function theorem, there exists $\eta=\eta(\xi)$ such that (4.10) holds locally on $\Gamma_{\text {shock }}$ near $\xi=\xi_{w}$. That is, there exists $\bar{\xi}>0$ such that $(\xi, \eta(\xi)) \in \Gamma_{\text {shock }}$ for $\xi_{w}<\xi \leq \bar{\xi}$.

Recall that $\eta^{\prime}(\xi)=f(\xi, \eta(\xi), \rho(\xi, \eta(\xi)))$. Then

$$
\eta^{\prime \prime}=f_{\xi}+f_{\eta} \eta^{\prime}+f_{\rho} \rho^{\prime} \quad \text { for } \xi \in\left(\xi_{w}, \bar{\xi}\right) \text {. }
$$

Notice that

$$
\eta^{\prime}(\xi)=f(\xi, \eta, \bar{c})=\frac{\xi \eta+\bar{c} \sqrt{\xi^{2}+\eta^{2}-\bar{c}^{2}}}{\xi^{2}-\bar{c}^{2}}
$$

then

$$
\begin{aligned}
f_{\xi} & =\frac{\eta}{\xi^{2}-\bar{c}^{2}}+\frac{\bar{c} \xi}{\left(\xi^{2}-\bar{c}^{2}\right) \sqrt{\xi^{2}+\eta^{2}-\bar{c}^{2}}}-\frac{2 \xi\left(\xi \eta+\bar{c} \sqrt{\xi^{2}+\eta^{2}-\bar{c}^{2}}\right)}{\left(\xi^{2}-\bar{c}\right)^{2}} \\
f_{\eta} & =\frac{\xi}{\xi^{2}-\bar{c}^{2}}+\frac{\eta \bar{c}}{\left(\xi^{2}-\bar{c}^{2}\right) \sqrt{\xi^{2}+\eta^{2}-\bar{c}^{2}}}
\end{aligned}
$$

Thus, we have

$$
\begin{aligned}
f_{\xi}+f_{\eta} \eta^{\prime}= & \frac{\eta}{\xi^{2}-\bar{c}^{2}}+\frac{\bar{c} \xi}{\left(\xi^{2}-\bar{c}^{2}\right) \sqrt{\xi^{2}+\eta^{2}-\bar{c}^{2}}}-\frac{2 \xi^{2} \eta}{\left(\xi^{2}-\bar{c}\right)^{2}}-\frac{2 \xi \bar{c} \sqrt{\xi^{2}+\eta^{2}-\bar{c}^{2}}}{\left(\xi^{2}-\bar{c}\right)^{2}} \\
& +\frac{\xi^{2} \eta}{\left(\xi^{2}-\bar{c}^{2}\right)^{2}}+\frac{\xi \bar{c} \sqrt{\xi^{2}+\eta^{2}-\bar{c}}}{\left(\xi^{2}-\bar{c}\right)^{2}}+\frac{\xi \eta^{2} \bar{c}}{\left(\xi^{2}-\bar{c}^{2}\right)^{2} \sqrt{\xi^{2}+\eta^{2}-\bar{c}^{2}}+\frac{\eta \bar{c}^{2}}{\left(\xi^{2}-\bar{c}\right)^{2}}} \\
= & \frac{\eta\left(\xi^{2}-\bar{c}^{2}-2 \xi^{2}+\xi^{2}+\bar{c}^{2}\right)}{\left(\xi^{2}-\bar{c}^{2}\right)}+\frac{\xi \bar{c}\left(\xi^{2}-\bar{c}^{2}-2 \xi^{2}-2 \eta^{2}+2 \bar{c}^{2}+\xi^{2}+\eta^{2}-\bar{c}^{2}+\eta^{2}\right)}{\left(\xi^{2}-\bar{c}^{2}\right)^{2} \sqrt{\xi^{2}+\eta^{2}-\bar{c}^{2}}} \\
= & 0 .
\end{aligned}
$$


Therefore, the sign of $\eta^{\prime \prime}$ is determined entirely by the sign of $f_{\rho}$ and $\rho^{\prime}$. Note that $\rho$ is increasing, $\rho^{\prime}>0$, and $\frac{d \bar{c}^{2}}{d \rho}>0$. Moreover, we have

$$
\begin{aligned}
\frac{\partial f}{\partial \bar{c}^{2}} & =\frac{-2 \xi \eta \bar{c} \sqrt{\xi^{2}+\eta^{2}-\bar{c}^{2}}+2 \eta^{2}\left(\xi^{2}+\eta^{2}-\bar{c}^{2}\right)+\left(\xi^{2}+\eta^{2}\right)\left(\bar{c}^{2}-\eta^{2}\right)}{\bar{c}\left(\xi \eta-\bar{c} \sqrt{\xi^{2}+\eta^{2}-\bar{c}^{2}}\right)^{2} \sqrt{\xi^{2}+\eta^{2}-\bar{c}^{2}}} \\
& =\frac{\left(\xi \bar{c}-\eta \sqrt{\xi^{2}+\eta^{2}-\bar{c}^{2}}\right)^{2}}{\bar{c}\left(\xi \eta-\bar{c} \sqrt{\xi^{2}+\eta^{2}-\bar{c}^{2}}\right)^{2} \sqrt{\xi^{2}+\eta^{2}-\bar{c}^{2}}} .
\end{aligned}
$$

If $\xi \eta \leq 0$, it is clear from (4.11) that $\frac{\partial f}{\partial \bar{c}^{2}}>0$.

If $\xi \eta>0$, from (4.11), we have

$$
\begin{aligned}
\frac{\partial f}{\partial \bar{c}^{2}} & =\frac{\left(\xi^{2}+\eta^{2}\right)^{2}\left(\bar{c}^{2}-\eta^{2}\right)^{2}\left(\xi \eta+\bar{c} \sqrt{\xi^{2}+\eta^{2}-\bar{c}^{2}}\right)^{2}}{\bar{c} \sqrt{\xi^{2}+\eta^{2}-\bar{c}^{2}}\left(\xi^{2}-\bar{c}^{2}\right)\left(\eta^{2}-\bar{c}^{2}\right)\left(\xi \bar{c}+\eta \sqrt{\xi^{2}+\eta^{2}-\bar{c}^{2}}\right)^{2}} \\
& =\frac{\left(\xi^{2}+\eta^{2}\right)^{2}\left(\xi \eta+\bar{c} \sqrt{\xi^{2}+\eta^{2}-\bar{c}^{2}}\right)^{2}}{\bar{c}\left(\bar{c}^{2}-\xi^{2}\right) \sqrt{\xi^{2}+\eta^{2}-\bar{c}^{2}}\left(\xi \bar{c}+\eta \sqrt{\xi^{2}+\eta^{2}-\bar{c}^{2}}\right)^{2}}>0 .
\end{aligned}
$$

These imply that $\eta=\eta(\xi)$ is strictly convex for $\xi \in\left[\xi_{w}, \xi_{1}\right)$.

Lemma 4.4 yields that problem (3.2)-(3.7) is equivalent to the following free boundary problem in the self-similar coordinates:

(i) Equation:

$$
L \rho=\sum_{i, j=1}^{2} D_{i}\left(a_{i j}(\xi, \eta, \rho) D_{j} \rho\right)+\sum_{i=1}^{2} b_{i}(\xi, \eta) D_{i} \rho=0 \quad \text { in } \Omega
$$

with

$$
\begin{aligned}
& a_{11}(\xi, \eta, \rho)=c^{2}(\rho)-\xi^{2}, \quad a_{22}(\xi, \eta, \rho)=c^{2}(\rho)-\eta^{2}, \\
& a_{12}(\xi, \eta, \rho)=a_{21}(\xi, \eta, \rho)=-\xi \eta, \quad b_{1}(\xi, \eta)=\xi, \quad b_{2}(\xi, \eta)=\eta .
\end{aligned}
$$

(ii) The shock equation:

$$
\frac{d \eta}{d \xi}=f(\xi, \eta, \rho)=\frac{\xi \eta+\bar{c} \sqrt{\xi^{2}+\eta^{2}-\bar{c}^{2}}}{\xi^{2}-\bar{c}^{2}} \quad \text { with } \eta\left(\xi_{1}\right)=\eta_{1}
$$

with the boundary condition on $\Gamma_{\text {shock}}$ :

$$
N \rho=\sum_{i=1}^{2} \beta_{i} D_{i} \rho=0 \quad \text { on } \Gamma_{\text {shock }}=\left\{\eta=\eta(\xi): 0 \leq \xi \leq \xi_{1}\right\},
$$

where $\beta_{1}$ and $\beta_{2}$ are the following functions of $(\xi, \eta), \rho$, and $\eta^{\prime}$ :

$$
\begin{aligned}
\beta_{1}= & \left(\xi^{2}+\eta^{2}\right)\left(-\eta^{\prime} \xi+\eta\right)\left(c^{2}(\rho)+\bar{c}^{2}\left(\rho, \rho_{0}\right)\right) \\
& -2 \bar{c}^{2}\left(\rho, \rho_{0}\right)\left(-\eta^{\prime} \xi\left(c^{2}+\eta^{2}\right)+\left(\eta-\eta\left(\eta^{\prime}\right)^{2}-\xi \eta^{\prime}\right)\left(c^{2}-\xi^{2}\right)\right)
\end{aligned}
$$


and

$$
\begin{aligned}
\beta_{2}= & \eta^{\prime}\left(\xi^{2}+\eta^{2}\right)\left(\eta-\eta^{\prime} \xi\right)\left(c^{2}(\rho)+\bar{c}^{2}\left(\rho, \rho_{0}\right)\right) \\
& -2 \bar{c}^{2}\left(\rho, \rho_{0}\right)\left(\left(\eta^{\prime} \eta-\xi-\xi\left(\eta^{\prime}\right)^{2}\right)\left(c^{2}-\eta^{2}\right)+\eta^{\prime} \eta\left(c^{2}+\xi^{2}\right)\right) .
\end{aligned}
$$

(iii) The remaining boundary conditions:

$$
\rho=\rho_{2} \text { on } \Gamma_{\text {sonic }}, \quad \rho_{\nu}=0 \text { on } \Gamma_{0}, \quad \rho\left(P_{2}\right)=\bar{\rho},
$$

where $\boldsymbol{\nu}$ is the outward normal to $\Omega$ at $\Gamma_{0}$.

It is easy to check that (4.13) is the oblique derivative boundary condition along $\Gamma_{\text {shock} \text {. }}$

With Lemma 4.4, we can show that Case 1 is the only case for the solutions, which implies that we can obtain the finer regularity near $P_{2}$.

Lemma 4.5. Suppose that $(\rho, r)$ is the solution to the free boundary problem (3.2)(3.7). Then the shock does not meet the circle $r=r_{0}$ at the wedge.

Proof. The main idea of the proof is the same as that in Lemma 4.3, and the only main difference is that the domain to be considered is a sector instead of a ball. We only list the major procedure and the difference here. We show our claim by contradiction. Otherwise, $r\left(\theta_{w}\right)=c_{0}$.

First, let $\eta=r \cos \left(\theta-\theta_{w}\right)$ and consider

$$
\phi=c_{0}^{2}+A_{1}\left(c_{0}-\eta\right)^{\frac{1}{2}}-B_{1}\left(c_{0}-\eta\right)^{\beta_{1}}+C_{1}\left(\theta-\theta_{0}\right)^{2},
$$

where $\theta \in\left[\theta_{w}, \theta_{w}+\delta\right], \delta>0$ small enough, $A_{1}, B_{1}, C_{1}>0$ and $\frac{1}{2}<\beta_{1}<1$, all of which will be specified later to prove that $\rho \in C^{\frac{1}{2}}$ near this boundary point.

Since $0 \leq c_{0}^{2}-\eta^{2}=\left(c_{0}^{2}-r^{2}\right)+r^{2} \sin ^{2}\left(\theta-\theta_{w}\right)$ on $\Gamma_{\text {shock }}$ from its convexity indicated in Lemma 4.4, we have

$$
0 \leq r^{2}-c_{0}^{2} \leq r^{2} \sin ^{2}\left(\theta-\theta_{w}\right) \leq C\left(\theta-\theta_{w}\right)^{2} \quad \text { on } \Gamma_{\text {shock }}
$$

for some constant $C>0$. This implies that $\bar{c}^{2}-c_{0}^{2} \leq r^{2}-c^{2} \leq r^{2}-c_{0}^{2} \leq C\left(\theta-\theta_{w}\right)^{2}$. Then

$$
c^{2}-c_{0}^{2} \leq C\left(\theta-\theta_{w}\right)^{2},
$$

since $c^{2}$ and $\bar{c}^{2}$ are both functions of $\rho$. We can choose $C_{1}>0$ so large that $c^{2} \leq \phi$ on $\Gamma_{\text {shock}}$. Then, as in the proof of Lemma 4.3, we can now show that $\phi$ is an upper barrier of $\rho$, i.e., $c^{2} \leq \phi$ in $N_{1}$, which implies

$$
0 \leq c^{2}-c_{0}^{2} \leq A_{1}\left(c_{0}-\eta\right)^{\frac{1}{2}}+C_{1}\left(\theta-\theta_{w}\right)^{2} .
$$

Next, for a lower barrier of $\rho$, as the proof of Lemma 4.3, we can show that there exist a neighborhood $N_{2}$ of $\left(r_{w}, \theta_{w}\right)$ and a constant $A_{2}>0$ such that

$$
c^{2}-c_{0}^{2} \geq A_{2}\left(c_{0}-r\right)^{\frac{1}{2}} \quad \text { in } N_{2} \cap\left\{(r, \theta): r \leq c_{0}\right\} .
$$

The only new here is the boundary $r=c_{0}$, which is obvious. This implies that

$$
a\left(c_{0}-r\right)^{\frac{1}{2}} \leq v:=\rho-\rho_{0} \leq A\left(c_{0}-r\right)^{\frac{1}{2}} \quad \text { in } N_{1} \cap N_{2} \cap V,
$$


where $V$ is an upward sector containing the wedge, with the vertex at $P_{2}$ and the angle smaller than $\frac{\pi}{2}$, for some constants $a$ and $A$ depending on $V$. This implies that the optimal regularity along the wedge is $C^{\frac{1}{2}}$ near the sonic circle.

With this optimal regularity in hand, we introduce the coordinates:

$$
x=c_{0}-r, y=\theta-\theta_{w}, v=c^{2}-c_{0}^{2} .
$$

Thus, rewriting the equation for $c^{2}$ in the divergence form, we have

$$
Q v=\left(a_{11}\left(v+2 c_{0} x-x^{2}\right) v_{x}\right)_{x}+b_{1} v_{x}+\left(a_{22} v_{y}\right)_{y}=0,
$$

where $a_{11}=\frac{c^{\frac{2(2-\gamma)}{\gamma-1}}}{\gamma-1}, a_{22}=\frac{c^{\frac{2}{\gamma-1}}}{\gamma-1} \frac{1}{r^{2}}$, and $b_{1}=\frac{c^{\frac{2}{\gamma-1}}}{\gamma-1} \frac{1}{r}$.

As in the proof of Lemma 4.3, scale $v$ in $N_{1} \cap N_{3} \cap V$ by defining

$$
u(S, T)=\frac{1}{S^{\frac{1}{5}}} v\left(S^{-\frac{12}{5}}, S^{-\frac{14}{5}} T\right)
$$

for $\left(S^{-\frac{12}{5}}, S^{-\frac{14}{5}} T\right) \in N_{1} \cap N_{3} \cap V$. Moreover, $u$ satisfies the governing equation (4.9).

From the optimal continuity, $0<a \leq S^{\frac{7}{5}} u \leq A$. Then, exactly following the proof of Lemma 4.3, we obtain a contradiction when $x_{0}$ is small. This completes the proof.

Finally, we establish the Lipschitz continuity for the solution near the degenerate sonic boundary.

Lemma 4.6. The solution $\rho$ to the free boundary problem (4.12)-(4.16) is Lipschitz continuous up to the boundary $\Gamma_{\text {sonic }}$.

Proof. On one hand, since $\rho \leq \rho_{1}$ in $\Omega$, we have

$$
c^{2}(\rho)-\xi^{2}-\eta^{2}<c^{2}\left(\rho_{1}\right)-\xi^{2}-\eta^{2} .
$$

On the other hand, it follows from Lemma 4.1 that

$$
c^{2}(\rho)-\xi^{2}-\eta^{2}>\xi^{2}+\eta^{2}-c^{2}\left(\rho_{1}\right) \quad \text { in } \Omega .
$$

Then we have

$$
\begin{aligned}
\left|c^{2}(\rho)-c^{2}\left(\rho_{1}\right)\right| & \leq\left|c^{2}(\rho)-\xi^{2}-\eta^{2}\right|+\left|c^{2}\left(\rho_{1}\right)-\xi^{2}-\eta^{2}\right| \\
& \leq 2\left|c^{2}\left(\rho_{1}\right)-\xi^{2}-\eta^{2}\right| \\
& \leq 4 c\left(\rho_{1}\right)\left|c\left(\rho_{1}\right)-\sqrt{\xi^{2}+\eta^{2}}\right|
\end{aligned}
$$

which implies that $\rho$ is Lipschiz continuous up to the degenerate boundary $\Gamma_{\text {sonic }}$.

Proof of the Existence Part of Theorem 2.1. The above seven lemmas, i.e., Lemmas 4.14.6, show that there exists a solution

$$
(\rho, r) \in C^{2+\alpha}(\Omega) \cap C^{\alpha}(\bar{\Omega}) \cap C^{0,1}\left(\Omega \cup \Gamma_{\text {sonic }}\right) \times C^{2+\alpha^{\prime}}\left(\left(\theta_{w}, \theta_{1}\right)\right) \cap C^{1,1}\left(\left[\theta_{w}, \theta_{1}\right]\right),
$$

which satisfies (2.6) $-(2.10)$. This completes the proof of the existence part. 


\section{Proof of Theorem 2.1: Optimal Regularity near the Sonic Boundary}

In this section, we prove that the Lipschitz continuity is the optimal regularity for $\rho$ across the sonic boundary $\Gamma_{\text {sonic }}$, as well as at the intersection point $P_{1}$ between $\Gamma_{\text {sonic }}$ and $\Gamma_{\text {shock. }}$. In $\S 4$, we have shown that the solution $\rho$ to the free boundary problem (4.12) - 4.16) is Lipschitz continuous in $\Omega$ up to the degenerate boundary $\Gamma_{\text {sonic }}$. Now we employ the approach introduced in Bae-Chen-Feldman [1] with the aid of the estimates in $\S 4$ to analyze the finer behavior of $\rho$ near the sonic circle $r=r_{1}:=c\left(\rho_{1}\right)$.

For $\varepsilon \in\left(0, \frac{c_{1}}{2}\right)$, we denote by

$$
\Omega_{\varepsilon}:=\Omega \cap\left\{(r, \theta): 0<c_{1}-r<\varepsilon\right\},
$$

the $\varepsilon$-neighborhood of the sonic circle $\Gamma_{\text {sonic }}$ within $\Omega$. In $\Omega_{\varepsilon}$, we introduce the coordinates:

$$
(x, y)=\left(c_{1}-r, \theta-\theta_{1}\right) .
$$

One of our main observations is that it is more convenient to study the regularity in terms of the difference between $c^{2}\left(\rho_{1}\right)$ and $c^{2}(\rho)$ :

$$
\psi:=c^{2}\left(\rho_{1}\right)-c^{2}(\rho),
$$

since $\psi$ and $\rho$ have the same regularity in $\Omega_{\varepsilon}$.

It follows from (1.13) that $\psi$ satisfies

$$
\begin{aligned}
\mathcal{L}_{1} \psi:= & \left(2 c_{1} x-\psi+O_{1}\right) \psi_{x x}+\left(c_{1}+O_{2}\right) \psi_{x}-\left(1+O_{3}\right) \psi_{x}^{2} \\
& +\left(1+O_{4}\right) \psi_{y y}-\left(\frac{1}{(\gamma-1) c_{1}^{2}}+O_{5}\right) \psi_{y}^{2}=0 \quad \text { in } Q_{r, R}^{+}
\end{aligned}
$$

in the $(x, y)$-coordinates, where

$$
\begin{array}{ll}
O_{1}(x, \psi)=-x^{2}, & O_{2}(x, \psi)=-3 x+\frac{\psi}{c_{1}}, \\
O_{3}(x, \psi)=-\frac{\gamma-2}{\gamma-1}\left(2 c_{1} x-\psi-x^{2}\right), & O_{4}(x, \psi)=\frac{c_{1}^{2}-\psi}{\left(c_{1}-x\right)^{2}}-1, \\
O_{5}(x, \psi)=\frac{1}{\left(c_{1}-x\right)^{2}}-\frac{1}{c_{1}^{2}} . &
\end{array}
$$

Moreover, $\psi$ satisfies

$$
\psi>0 \quad \text { in } Q_{r, R}^{+}
$$

and the following Dirichlet boundary condition:

$$
\psi=0 \quad \text { on } \partial Q_{r, R}^{+} \cap\{x=0\},
$$

where $Q_{r, R}^{+}:=\{(x, y): x \in(0, r),|y|<R\} \subset \mathbb{R}^{2}$, with $R=\theta_{w}-\theta_{1}$, since we can extend $\psi(x, y)$ from $\Omega_{\varepsilon}$, by defining $\psi(x, y)=\psi(x,-y)$ for $(x, y) \in \Omega_{\varepsilon}$, and extend the domain $\Omega_{\varepsilon}$ with respect to $y$. Thus, without further comment, we study the behavior of $\psi$ in $Q_{r, R}^{+}$.

It is easy to see that the terms $O_{i}(x, y), i=1, \cdots, 5$, are continuously differentiable and

$$
(5.7) \frac{\left|O_{1}(x, y)\right|}{x^{2}}+\frac{\left|O_{k}(x, y)\right|}{x}+\frac{\left|D O_{1}(x, y)\right|}{x}+\left|D O_{k}(x, y)\right| \leq N \quad \text { for } k=2, \cdots, 5,
$$


in $\{x>0\}$ for some constant $N$ depending only on $c_{1}$ and $\gamma$. Inequality (5.7) implies that the terms $O_{i}(x, y), i=1, \cdots, 5$, are small. Thus, the main terms of (5.3) form the following equation:

$$
\left(2 c_{1}-\psi\right) \psi_{x x}+c_{1} \psi_{x}-\psi_{x}^{2}+\psi_{y y}-\frac{1}{(\gamma-1) c_{1}^{2}} \psi_{y}^{2}=0 \quad \text { in } Q_{r, R}^{+} .
$$

It follows from Lemmas 4.1 and 4.5 that

$$
0 \leq \psi \leq 2\left(c_{1}-\vartheta\right) x
$$

where $\vartheta$ depends only on $\rho_{1}$ and $\gamma$. Then equation (5.8) is uniformly elliptic in every subdomain $\{x>\delta\}$ with $\delta>0$. The same is true for (5.3) in $Q_{r, R}^{+}$if $r$ is sufficiently small.

Remark 5.1. If $\hat{r}$ is sufficiently small, depending only on $c_{1}$ and $\gamma$, then (5.7) and (5.9) imply that (5.3) is uniformly elliptic with respect to $\psi$ in $Q_{r, R}^{+} \cap\{x>\delta\}$ for any $\delta \in\left(0, \frac{\hat{r}}{2}\right)$. We will always assume such a choice of $\hat{r}$ hereafter.

5.1. First-order lower bound of $\psi$. In order to prove that $C^{0,1}$ is the optimal regularity of $\psi$ across the sonic boundary, our idea is to construct a positive subsolution of (5.3) and (5.5) -(5.6) first, which provides our desired lower bound of $\psi$.

Lemma 5.2. Let $\psi$ be a solution of the Dirichlet problem (5.3) and (5.5)-(5.6) . Then there exist $\hat{r}>0$ and $\mu>0$, depending only on $c_{1}, \gamma, \theta_{w}$, and $\inf _{Q_{\hat{r}, R}^{+} \cap\{x>\hat{r} / 2\}} \psi$, such that, for all $r \in\left(0, \frac{\hat{r}}{2}\right]$,

$$
\psi(x, y) \geq \mu c_{1} x \quad \text { in } Q_{r, \frac{15 R}{16}}^{+} .
$$

Proof. In the proof below, without further comment, all the constants depend only on the data, i.e., $c_{1}, \hat{r}, \gamma, \theta_{w}$, and $\inf _{Q_{\hat{r}, R}^{+} \cap\{x>\hat{r} / 2\}} \psi$, unless otherwise is stated.

Fix $y_{0}$ with $\left|y_{0}\right| \leq \frac{15 R}{16}$. We now prove that

$$
\psi\left(x, y_{0}\right) \geq \frac{5}{8} \mu x \quad \text { for } x \in(0, r) .
$$

Without loss of generality, we may assume that $R=2$ and $y_{0}=0$; otherwise, we set $\tilde{\psi}(x, y)=\psi\left(x, y_{0}+\frac{R}{32} y\right)$ for all $(x, y) \in Q_{\hat{r}, 2}^{+}$. Then $\tilde{\psi}(x, y) \in C\left(\overline{Q_{\hat{r}, R}^{+}}\right) \cap C^{2}\left(Q_{\hat{r}, R}^{+}\right)$ satisfies (5.3) with (5.7) and (5.9) in $Q_{\hat{r}, 2}^{+}$, with some modified constants $N$, $\vartheta$, and $O_{i}$, depending only on the corresponding quantities in the original equation and on R. Moreover,

$$
\inf _{Q_{\hat{r} 2}^{+} \cap\{x>\hat{r} / 2\}} \tilde{\psi}=\inf _{Q_{\hat{r}, R}^{+} \cap\{x>\hat{r} / 2\}} \psi
$$

Then (5.11) for $\psi$ follows from (5.11) for $\tilde{\psi}$ with $y_{0}=0$ and $R=2$. Thus we keep the original notation with $y_{0}=0$ and $R=2$. That is, it suffices to prove that

$$
\psi(x, 0) \geq \frac{5}{8} \mu x \quad \text { for } x \in(0, r) .
$$


By the Harnack inequality, we conclude that, for any $r \in\left(0, \frac{\hat{r}}{2}\right)$, there exists $\sigma=\sigma(r)>0$, depending only on $r$ and the data $c_{1}, \hat{r}, \gamma, \theta_{w}$, and $\inf _{Q_{\hat{r}, R}^{+} \cap\{x>\hat{r} / 2\}} \psi$, such that

$$
\psi \geq \sigma \quad \text { on } Q_{\hat{r}, 3 / 2}^{+} \cap\{x>r\} .
$$

Let $r \in\left(0, \frac{\hat{r}}{2}\right)$ and

$$
0<\mu_{0} \leq \min \left\{\frac{\sigma(r)}{r}, c_{1}\right\}
$$

where $r$ will be chosen later. Define

$$
g(y)= \begin{cases}\mu(y+1)^{2}, & -1 \leq y<-\frac{1}{2} \\ \mu\left(2 y^{4}-2 y^{2}+\frac{5}{8}\right), & -\frac{1}{2} \leq y \leq \frac{1}{2} \\ \mu(y-1)^{2}, & \frac{1}{2}<y \leq 1\end{cases}
$$

Set $w(x, y)=\mu x g(y)$ with $g \in C^{2}([-1,1])$. Then, using (5.14) and (5.15), we obtain that, for all $x \in(0, r)$ and $|y|<1$,

$$
\left\{\begin{array}{l}
w(0, y)=0 \leq \psi(0, y) \\
w(r, y) \leq \frac{5}{8} \mu r \leq \psi(r, y) \\
w(x, \pm 1)=0 \leq \psi(x, \pm 1)
\end{array}\right.
$$

Therefore, we have

$$
w \leq \psi \quad \text { on } \partial Q_{r, 1}^{+} .
$$

Next, we show that $w(x, y)$ is a strict subsolution $\mathcal{L}_{1} w(x, y)>0$ in $Q_{r, 1}^{+}$, if the parameters are appropriately chosen. In fact,

$$
\begin{aligned}
& \mathcal{L}_{1} w(x, y) \\
& =\left(c_{1} g(y)-g^{2}(y)\right) \\
& \quad+x\left(g^{\prime \prime}(y)-\frac{1}{(\gamma-1) c_{1}^{2}} x\left(g^{\prime}(y)\right)^{2}+\frac{O_{2}}{x} g(y)-\frac{O_{3}}{x} g^{2}(y)+O_{4} g^{\prime}(y)-x O_{5}\left(g^{\prime}(y)\right)^{2}\right) .
\end{aligned}
$$

On one hand, for $1-|y|<\varepsilon_{0}$ with $\varepsilon_{0}$ small enough, we can see

$$
\begin{aligned}
& g^{\prime \prime}(y)-\frac{1}{(\gamma-1) c_{1}^{2}} x\left(g^{\prime}(y)\right)^{2}+\frac{O_{2}}{x} g(y)-\frac{O_{3}}{x} g^{2}(y)+O_{4} g^{\prime}(y)-x O_{5}\left(g^{\prime}(y)\right)^{2} \\
& \geq g^{\prime \prime}(y)-\frac{1}{(\gamma-1) c_{1}^{2}} x\left(g^{\prime}(y)\right)^{2}-N x(g(y)+1) g(y)-x N g^{\prime}(y)+N x^{2}\left(g^{\prime}(y)\right)^{2} \\
& =: h(x, y) .
\end{aligned}
$$

It is easy to see that $h(x, y)$ is continuous with respect to $x, h(0, y)=0$, and that there exists $r_{1}>0$ such that $h(x, y)>0$ for $r<r_{1}$. 
On the other hand, for $1-|y|>\varepsilon_{0}$,

$$
\begin{aligned}
& \mathcal{L}_{1} w(x, y) \\
& \geq x\left(g^{\prime \prime}(y)-\frac{1}{(\gamma-1) c_{1}^{2}} x\left(g^{\prime}(y)\right)^{2}+\frac{O_{2}}{x} g(y)-\frac{O_{3}}{x} g^{2}(y)+O_{4} g^{\prime}(y)-x O_{5}\left(g^{\prime}(y)\right)^{2}\right) \\
& \quad+\mu \varepsilon_{0}^{2}\left(c_{1}-\frac{5}{8} \mu\right) .
\end{aligned}
$$

Then there exists $r_{2}>0$ such that the above inequality is positive.

We claim

$$
\sup _{Q_{r, 1}^{+}}(w-\psi) \leq \sup _{\partial Q_{r, 1}^{+}}(w-\psi) \leq 0,
$$

whenever $0<r<r_{0}:=\min \left\{r_{1}, r_{2}\right\}$ and $\mu \in\left(0, \mu_{0}\right]$. Otherwise, there exists a point $\left(x_{0}, y_{0}\right) \in Q_{r, 1}^{+}$such that

$$
\begin{aligned}
0< & \left(\mathcal{L}_{1} w-\mathcal{L}_{1} \psi\right)\left(x_{0}, y_{0}\right) \\
= & \left(2 c_{1} x-\psi+O_{1}\right)(w-\psi)_{x x}+\left(c_{1}+O_{2}\right)(w-\psi)_{x}-\left(1+O_{3}\right)(w+\psi)_{x}(w-\psi)_{x} \\
& +\left(1+O_{4}\right)(w-\psi)_{y y}-\left(\frac{1}{(\gamma-1) c_{1}^{2}}+O_{5}\right)(w+\psi)_{y}(w-\psi)_{y} \leq 0,
\end{aligned}
$$

where we have used the fact that $w_{x x}=0$, which is a contradiction. Hence, we obtain our claim:

$$
\psi(x, y) \geq w(x, y)=x f(y) \quad \text { in } Q_{r, 1}^{+}
$$

In particular,

$$
\psi(x, 0) \geq \frac{5}{8} \mu x \quad \text { for } \quad x \in[0, r] .
$$

This implies (5.11). Then (5.10) holds by modifying $\mu$, which is still denoted by $\mu$. This completes the proof.

5.2. $C^{1, \alpha}$-Estimate of $\psi$. If $\psi$ satisfies (5.3), (5.5)-(5.6), and (5.9), it is expected that $\psi$ is very close to $c_{1} x$, which is a solution of (5.8). More precisely, we now prove

$$
\left|\psi(x, y)-c_{1} x\right| \leq C x^{1+\alpha} \quad \text { for all }(x, y) \in Q_{\hat{r}, \frac{7 R}{8}}^{+}
$$

for some constant $C$.

To prove this, we study the function:

$$
W(x, y):=c_{1} x-\psi(x, y) .
$$

By (5.3), $W$ satisfies

$$
\begin{array}{cl}
\mathcal{L}_{2} W=\left(c_{1} x+W+O_{1}\right) W_{x x}-\left(c_{1}-O_{2}-2 c_{1} O_{3}\right) W_{x}+\left(1-O_{3}\right) W_{x}^{2} \\
\quad+\left(1+O_{4}\right) W_{y y}-\left(\frac{1}{(\gamma-1) c_{1}^{2}}-O_{5}\right) W_{y}^{2} \\
=c_{1} O_{2}+c_{1}^{2} O_{3} & \text { in } Q_{\hat{r}, R}^{+}, \\
W(0, y)=0 & \text { on } \partial Q_{\hat{r}, R}^{+} \cap\{x=0\}, \\
-\left(c_{1}-\vartheta\right) x \leq W(x, y) \leq c_{1} x & \text { in } Q_{\hat{r}, R}^{+} .
\end{array}
$$

Then we establish the following two estimates. 
Proposition 5.3. Let $c_{1}, \hat{r}, R$, and $\vartheta$ be the same as in Lemma 5.2. Then, for any $\alpha \in(0,1)$, there exist positive constants $r$ and $A$, which depend only on $N, c_{1}, \hat{r}, R$, $\vartheta$, and $\alpha$, such that, if $W \in C\left(\overline{Q_{\hat{r}, R}^{+}}\right) \cap C^{2}\left(Q_{\hat{r}, R}^{+}\right)$satisfies (5.17)-(15.19), then

$$
W(x, y) \leq A x^{1+\alpha} \quad \text { in } Q_{r, \frac{3 R}{4}}^{+} .
$$

Proof. The main idea of the proof is the same as that in [1], and we only list the major procedure and the difference here.

First, we prove that there exist $\alpha_{1} \in\left(0, \frac{1}{2}\right)$ and $r_{1}>0$ such that, if $W \in C\left(\overline{Q_{\hat{r}, R}^{+}}\right) \cap$ $C^{2}\left(Q_{\hat{r}, R}^{+}\right)$satisfies (5.17)-(5.19), then

$$
W(x, y) \leq \frac{c_{1}\left(1-\mu_{1}\right)}{r^{\alpha}} x^{1+\alpha} \quad \text { in } Q_{r, \frac{7 R}{8}}^{+},
$$

whenever $\alpha \in\left(0, \alpha_{1}\right], r \in\left(0, r_{1}\right]$, and $\mu_{1}<\min \left\{\mu, \frac{1}{2}\right\}$, where $\mu$ is the constant determined by Lemma 5.2 .

As in [1, we first note that, without loss of generality, we may assume that $R=2$ and $y_{0}=0$. Then it suffices to prove that

$$
W(x, 0) \leq \frac{c_{1}\left(1-\mu_{1}\right)}{r^{\alpha}} x^{1+\alpha} \quad \text { for } x \in(0, r)
$$

for some $r \in\left(0, r_{0}\right)$ and $\alpha \in\left(0, \alpha_{1}\right)$, under the assumptions that (5.17)-(5.19) hold in $Q_{\hat{r}, 2}^{+}$. For any given $r \in\left(0, r_{0}\right)$, let

$$
v=A_{1} x^{1+\alpha}\left(1-y^{2}\right)+B_{1} x y^{2}
$$

with $A_{1} r=c_{1}\left(1-\mu_{1}\right)$ and $B_{1}=c_{1}\left(1-\mu_{1}\right)$. Then we obtain

$$
W \leq v \quad \text { on } \partial Q_{r_{0}, 1}^{+},
$$

and

$$
\mathcal{L}_{2} v-\mathcal{L}_{2} W-v_{x x}(v-W)<0 \quad \text { in } Q_{r, 1}^{+},
$$

whenever $r \in\left(0, r_{1}\right]$ and $\alpha \in\left(0, \alpha_{1}\right]$ so that

$$
(2 \alpha-1)(\alpha+1) c_{1} A_{1}<-\frac{\mu_{1}}{2},
$$

and

$$
r_{1}<\min \left\{\left(\frac{\mu_{1}}{4 c_{1}}\right)^{\frac{1}{\alpha}},\left(\frac{B_{1} c_{2}-B_{1}^{2}}{C}\right)^{\frac{1}{\alpha}}, r_{0}\right\}
$$

Then

$$
W \leq v \quad \text { in } Q_{r, 1}^{+} .
$$

Next, we generalize the result for any $\alpha \in(0,1)$, which suffices to show that for the case $\alpha>\alpha_{1}$. Fix any $\alpha \in\left(\alpha_{1}, 1\right)$ and set the following comparison function:

$$
v=\frac{c_{1}\left(1-\mu_{1}\right)}{r_{1}^{\alpha_{1}} r^{\alpha-\alpha_{1}}} x^{1+\alpha}\left(1-y^{2}\right)+\frac{c_{1}\left(1-\mu_{1}\right)}{r_{1}^{\alpha_{1}}} x^{1+\alpha_{1}} y^{2} .
$$

Then, as before, we can prove

$$
W \leq v \quad \text { on } \partial Q_{r, 1}^{+} \quad \text { for } r \in\left(0, r_{1}\right]
$$


and

$$
\mathcal{L}_{2} v-\mathcal{L}_{2} W-v_{x x}(v-W)<0 .
$$

Then it is easy to prove that this proposition holds with

$$
A=\frac{c_{1}\left(1-\mu_{1}\right)}{r_{1}^{\alpha_{1}} r^{\alpha-\alpha_{1}}} .
$$

Proposition 5.4. Let $c_{1}, \hat{r}, R, \vartheta$, and $O_{i}$ be the same as in Lemma 5.2. Then, for any $\alpha \in(0,1)$, there exist positive constants $r$ and $B$, depending on $N, c_{1}, \hat{r}, R, \vartheta$, and $\alpha$, so that, if $W \in C\left(\overline{Q_{\hat{r}, R}^{+}}\right) \cap C^{2}\left(Q_{\hat{r}, R}^{+}\right)$satisfies (5.17)-(5.19), we have

$$
W(x, y) \geq-B x^{1+\alpha} \quad \text { in } Q_{r, \frac{3 R}{4}}^{+} .
$$

Proof. Similar to the proof of Proposition 5.3, it suffices to prove that, with the assumption $R=2$,

$$
W(x, 0) \geq-\frac{c_{1}-\vartheta}{r^{\alpha}} x^{1+\alpha} \quad \text { for } x \in(0, r)
$$

for some $r>0$ and $\alpha \in\left(0, \alpha_{2}\right)$. For this, we use the comparison function:

$$
v(x, y):=-L x^{1+\alpha}\left(1-y^{2}\right)-K x y^{2}, \quad \text { with } L r^{\alpha}=K=\frac{c_{1}-\vartheta}{r^{\alpha}} .
$$

It is easy to check that

$$
W \geq v \quad \text { on } \partial Q_{r, 1}^{+} \quad \text { for } r \in\left(0, r_{1}\right] .
$$

Then we follow the same procedure as in [1], except that $\mathcal{L}_{2} v>\mathcal{L}_{2} W$, to find that the conditions for the choice of $\alpha, r>0$ are inequalities (5.21) and (5.22) with $\left(\mu_{1}, r_{1}\right)$ replaced by $\left(\beta, r_{2}\right)$, respectively, and with an appropriate constant $C$.

We claim that

$$
\min _{Q_{r, 1}^{+}}(W-v) \geq \min _{\partial Q_{r, 1}^{+}}(W-v) \geq 0
$$

Otherwise, there exists a point $\left(x_{0}, y_{0}\right) \in Q_{r, 1}^{+}$such that $(W-v)\left(x_{0}, y_{0}\right)<0$ and

$$
\begin{aligned}
0> & \left(\mathcal{L}_{2} W-\mathcal{L}_{2} v\right)\left(x_{0}, y_{0}\right) \\
= & \left(c_{1} x+W+O_{1}\right)(W-v)_{x x}-\left(c_{1}-O_{2}-2 c_{1} O_{3}\right)(W-v)_{x} \\
& +\left(1-O_{3}\right)(W+v)_{x}(v-W)_{x}+\left(1+O_{4}\right)(W-v)_{y y} \\
& -\left(\frac{1}{(\gamma-1) c_{1}^{2}}-O_{5}\right)(W+v)_{x}(W-v)_{x}+v_{x x}(W-v) \\
\geq & 0 \quad \operatorname{in} Q_{r, 1}^{+},
\end{aligned}
$$

which is a contradiction. This completes the proof for the case $\alpha \leq \alpha_{2}$.

For the case $\alpha \in\left(\alpha_{2}, 1\right)$, we set the comparison function:

$$
u_{-}(x, y):=-\frac{c_{1}-\vartheta}{r_{2}^{\alpha_{2}} r^{\alpha-\alpha_{2}}} x^{1+\alpha}\left(1-y^{2}\right)-\frac{c_{1}-\vartheta}{r_{2}^{\alpha_{2}}} x^{1+\alpha_{2}} .
$$


Then, using the argument as before, we can choose $r>0$ appropriately small such that

$$
\mathcal{L}_{2} u_{-}-\mathcal{L}_{2} W>0
$$

holds for all $(x, y) \in Q_{r, 1}^{+}$.

Lemma 5.5. Let $\psi \in C\left(\overline{Q_{\hat{r}, R}^{+}}\right) \cap C^{2}\left(Q_{\hat{r}, R}^{+}\right)$be a solution of the Dirichlet problem (5.3) and (5.5) -(5.6). Then $\psi \in C^{1, \alpha}\left(\overline{Q_{\hat{r} / 2, R / 2}^{+}}\right)$for any $\alpha \in(0,1)$ with

$$
\psi_{x}(0, y)=c_{1}, \quad \psi_{y}(0, y)=0 \quad \text { for any }|y| \leq \frac{R}{2} .
$$

Proof. The proof is quite similar to that in [1, and the main difference is the scaling due to the different equations. For fixed $z_{0}=\left(x_{0}, y_{0}\right) \in Q_{r / 2, R / 2}^{+}$, rescale $W$ in $R_{z_{0}}$ by defining

$$
W^{\left(z_{0}\right)}(S, T)=\frac{1}{x_{0}^{1+\alpha}} W\left(x_{0}+\frac{x_{0}}{8} S, y_{0}+\frac{\sqrt{x_{0}}}{8} T\right) \quad \text { for }(S, T) \in Q_{1},
$$

where $Q_{h}=(-h, h)^{2}$ for $h>0$. Keep this in mind, we can prove this lemma easily by following [1] step by step. Thus we omit the detail of proof here.

Now, following the procedure in [1] step by step with the aid of the results above, we can obtain the next theorem.

Theorem 5.6. Let $\rho \in C^{2+\alpha}(\Omega) \cap C(\bar{\Omega})$ be the solution of the free boundary problem (2.6) -(2.10) in $\S 4$. Then $\rho$ cannot be $C^{1}$ across the degenerate sonic boundary $\Gamma_{\text {sonic }}$.

We now study more detailed regularity of $\rho$ near the sonic circle. From now on, we use a localized version of $\Omega_{\varepsilon}$ : For a given neighborhood $\mathcal{N}\left(\Gamma_{\text {sonic }}\right)$ of $\Gamma_{\text {sonic }}$ and $\varepsilon>0$, define

$$
\Omega_{\varepsilon}:=\Omega \cap \mathcal{N}\left(\Gamma_{\text {sonic }}\right) \cap\{x<\varepsilon\} .
$$

Since $\mathcal{N}\left(\Gamma_{\text {sonic }}\right)$ is fixed in the following theorem, we do not specify the dependence of $\Omega_{\varepsilon}$ on $\mathcal{N}\left(\Gamma_{\text {sonic }}\right)$.

Finally, we show the regularity part of Theorem 2.1 .

Theorem 5.7. Let $\rho$ be the solution of the free boundary problem (4.12)-(4.16) established in $\S 4$ and satisfy the properties: There exists a neighborhood $\mathcal{N}\left(\Gamma_{\text {sonic }}\right)$ of $\Gamma_{\text {sonic }}$ such that, for $\psi:=c_{1}^{2}-c^{2}(\rho)$,

(a) $\psi$ is $C^{0,1}$ across part $\Gamma_{\text {sonic }}$ of the degenerate sonic boundary;

(b) there exists $\vartheta_{0}>0$ so that, in the coordinates (5.1),

$$
|\psi| \leq\left(2 c_{1}-\vartheta_{0}\right) x \quad \text { in } \Omega \cap \mathcal{N}\left(\Gamma_{\text {sonic }}\right) .
$$

Then we have 
(i) There exists $\varepsilon_{0}>0$ such that $\psi$ is $C^{1, \alpha}$ in $\Omega$ up to $\Gamma_{\text {sonic }}$ away from point $P_{1}$ for any $\alpha \in(0,1)$. That is, for any $\alpha \in(0,1)$ and $\left(\xi_{0}, \eta_{0}\right) \in \overline{\Gamma_{\text {sonic }}} \backslash P_{1}$, there exists $K<\infty$ depending only on $\rho_{0}, \rho_{1}, \gamma, \varepsilon_{0}, \alpha,\|\psi\|_{C^{0,1}}$, and $d=$ $\operatorname{dist}\left(\left(\xi_{0}, \eta_{0}\right), \Gamma_{\text {sonic }}\right)$ so that

$$
\|\psi\|_{1, \alpha ; \overline{B_{d / 2}}\left(\xi_{0}, \eta_{0}\right) \cap \Omega_{\varepsilon_{0} / 2} \leq K} \leq
$$

(ii) For any $\left(\xi_{0}, \eta_{0}\right) \in \Gamma_{\text {sonic }} \backslash P_{1}, \lim _{\substack{\xi, \eta) \rightarrow\left(\xi_{0}, \eta_{0}\right) \\(\xi, \eta) \in \Omega}} D_{r} \psi=c_{1}$;

(iii) The limit $\lim _{\substack{\xi, \eta) \rightarrow P_{1} \\(\xi, \eta) \in \Omega}} D_{r} \psi$ does not exist. $(\xi, \eta) \in \Omega$

The proof is quite similar to the one in [1], which can be achieved by following the proof of Theorem 4.2 in [1] step by step with the aid of the estimates obtained above. Hence we omit the proof here.

\section{Proof of Theorem 2.1: Global Solutions}

Finally, we show that the solution established above is a global solution indeed, valid through the sonic circle $\Gamma_{\text {sonic }}$, as claimed in Theorem 2.1.

Since $\rho$ is only Lipschitz continuous across the sonic circle, we treat the solution in the weak sense: For every $\zeta \in C_{c}^{\infty}\left(\Omega_{-}\right)$, with $\Omega_{-}$denoting the region of the left state,

$$
\int_{\Omega_{-}}\left(\left(c^{2}-r^{2}\right) \rho_{r} \zeta_{r}+\frac{c^{2}}{r^{2}} \rho_{\theta} \zeta_{\theta}-\frac{c^{2}}{r} \rho_{r} \zeta\right) \mathrm{d} r \mathrm{~d} \theta=0 .
$$

Notice that $\rho$ is Lipschitz continuous across the sonic circle. Then, due to the Green theorem, the integrand is equal to 0 if and only if

$$
\left[\left(\left(c^{2}-r^{2}\right) \rho_{r}, \frac{c^{2}}{r^{2}} \rho_{\theta}\right) \cdot \boldsymbol{\nu}\right]=0 \quad \text { on } \Gamma_{\text {sonic }},
$$

where the bracket [.] denotes the difference of the quantity between two sides of the sonic circle, and $\boldsymbol{\nu}$ is the normal direction. It is obvious because from the facts that $\left(\rho_{r}, \rho_{\theta}\right)=\left(-c_{1}, 0\right)$ up to the sonic circle from the subsonic domain obtained in Lemma 5.5, $\left(\rho_{r}, \rho_{\theta}\right)=(0,0)$ from the supersonic domain and the fact that $c^{2}-r^{2}=0$ on the sonic circle. This completes the proof of Theorem 2.1.

\section{Existence and Regularity of Global Solutions of the Nonlinear WAVE SYSTEM}

In our main theorem, Theorem [2.1, we have constructed a global solution $\rho$ of the second-order equation (4.12) in $\Omega$, combining this function with $\rho=\rho_{1}$ in state (1) and $\rho=\rho_{0}$ in state (0). That is, we have obtained the global density function $\rho$ that is piecewise constant in the supersonic region, which is Lipschitz continuous across the degenerate sonic boundary $\Gamma_{\text {sonic }}$ from $\Omega$ to state (1). 
To recover the momentum components, $m$ and $n$, we can integrate the second and third equation in (1.7). These can be also written in the radial variable $r$,

$$
\frac{\partial m}{\partial r}=\frac{1}{r} p(\rho)_{\xi}, \quad \frac{\partial n}{\partial r}=\frac{1}{r} p(\rho)_{\eta},
$$

and integrated from the boundary of the subsonic region toward the origin.

Note that we have proved that the limit of $D \rho$ does not exist at $P_{1}$ as $(\xi, \eta)$ in $\Omega$ tends to $\left(\xi_{1}, \eta_{1}\right)$, but $|D c(\rho)|$ has a upper bound. Thus, $p(\rho)$ is Lipschitz, which implies that $(m, n)$ are at least Lipschitz across the sonic circle $\Gamma_{\text {sonic }}$.

Furthermore, $(m, n)$ have the same regularity as $\rho$ inside $\Omega$ except the origin $r=0$. However, $(m, n)$ may be multi-valued at the origin $r=0$.

In conclusion, we have

Theorem 7.1. Let the wedge angle $\theta_{w}$ be between $-\pi$ and 0 . Then there exists a global solution $(\rho, m, n)(r, \theta)$ with the free boundary $r=r(\theta), \theta \in\left[\theta_{w}, \theta_{1}\right]$, of Problem 2 such that

$$
(\rho, m, n) \in C^{2+\alpha}(\Omega), \quad \rho \in C^{\alpha}(\bar{\Omega}), \quad r \in C^{2+\alpha}\left(\left[\theta_{w}, \theta_{1}\right)\right) \cap C^{1,1}\left(\left[\theta_{w}, \theta_{1}\right]\right),
$$

and $(\rho, m, n)=\left(\rho_{1}, m_{1}, 0\right)$ in the domain $\left\{\xi<\xi_{1}, r>r_{1}\right\}$ and $\left(\rho_{0}, 0,0\right)$ in the domain $\left\{\xi>\xi_{1}, \eta>\eta_{1}\right\} \cup\left\{r>r(\theta), \theta \in\left[\theta_{w}, \theta_{1}\right]\right\}$. Moreover, the solution $(\rho, m, n)(r, \theta)$ with the free boundary $r=r(\theta)$ satisfies the following properties:

(i) $\rho>\rho_{0}$ on the shock $\Gamma_{\text {shock, }}$, that is, the shock $\Gamma_{\text {shock }}$ is separated from the sonic circle $C_{0}$ of state (0);

(ii) The shock $\Gamma_{\text {shock }}$ is convex in the self-similar coordinates $(\xi, \eta)$ and strictly convex up to point $P_{1}$, except point $P_{2}$;

(iii) The solution $(\rho, m, n)$ is $C^{1, \alpha}$ up to $\Gamma_{\text {sonic }}$ and Lipschitz continuous across $\Gamma_{\text {sonic }}$;

(iv) The Lipschitz regularity of the solution across $\Gamma_{\text {sonic }}$ and at $P_{1}$ from the inside is optimal;

(v) The momentum components $(m, n)$ may be multi-valued at the origin.

\section{Appendix: Proof of Lemma 3.6}

For self-containedness, we illustrate a stretched proof of Lemma 3.6 in the following:

Proof. For the notational simplicity, we write $\rho=\rho^{\varepsilon, \delta}$ throughout the proof.

1. The existence part of the proof is similar to that in [4. The main idea is that, for any function $w \in \mathcal{W}$, we define a mapping

$$
T: \mathcal{W} \subset C_{\left(-\gamma_{1}\right)}^{2} \rightarrow C_{\left(-\gamma_{1}\right)}^{2}
$$

by $T w=\rho$, where $\rho$ is the solution to the linear regularized fixed boundary problem (3.12) - 3.13) solved in Lemma 3.5. By Lemma 3.5. $T$ obviously maps $\mathcal{W}$ into a bounded set in $C_{\left(-\gamma_{V}\right)}^{2+\alpha}$, where $\gamma_{V}$ is the value given by Lemma 3.5. Since $\gamma_{V}$ is independent of $\gamma_{1}$, we may take $\gamma_{1}=\frac{\gamma_{V}}{2}$ so that $T(\mathcal{K})$ is precompact in $C_{\left(-\gamma_{1}\right)}^{2}$. 
Next, it is easy to verify that $T w$ satisfies (W1) and (W3) in Definition 3.2 by the boundary conditions, the maximum principle, and the standard interior and boundary Hölder estimates ( $c f$. Theorems 8.22 and 8.27 in [11]). In order to show that $T$ maps $\mathcal{W}$ into itself, the remaining task is to show that $T w$ satisfies (W2) in Definition 3.2 . To achieve this, it suffices to find $K>0$ such that

$$
\sup _{\delta>0}\left(\delta^{2-\gamma_{1}}\|\rho\|_{2, \bar{\Omega} \backslash\left\{\Gamma(\delta) \cup \Omega_{V}(\delta)\right\}}\right)<K,
$$

under the assumption that $\|w\|_{2}^{\left(-\gamma_{1}\right)} \leq K$. Note that Lemma 3.4 gives us a local bound for the weighted norm of $\rho$ on $\Gamma\left(d_{0}\right)$ of the form

$$
d^{2-\gamma_{1}}\|\rho\|_{2} \leq d^{1-\gamma_{1}+\mu} C,
$$

which holds for all $d<d_{0}$, where $C$ depends on $K, \alpha_{1}$, and $\gamma_{1}$. To show (17.2), we make the $L^{\infty}$-estimate by considering separately the domains in $\bar{\Omega} \backslash\left\{\Gamma(\delta) \cup \Omega_{V}(\delta)\right\}$ for which $\delta>\tilde{d}$, with $\tilde{d} \leq d_{0}$ to be specified later, and the domains for which $\delta \leq \tilde{d}$.

In the domains of the first kind, $\bar{\Omega} \backslash\left\{\Gamma(\delta) \cup \Omega_{V}(\delta)\right\}$ with $\delta>\tilde{d}$, the solution is smooth, and trivially its $C^{2}$-norm bound is independent of $K$ by the uniform Hölder estimate, the interpolation inequality ( $c f$. Lemma 6.32, [11]), and the bootstrap iteratively.

Finally, we estimate $\delta^{2-\gamma_{1}}\|\rho\|_{2, \bar{\Omega} \backslash\left\{\Gamma(\delta) \cup \Omega_{V}(\delta)\right\}}$ with $\delta \leq \tilde{d}$. We divide the subdomain $\bar{\Omega} \backslash\left\{\Gamma(\delta) \cup \Omega_{V}(\delta)\right\}$ into two parts: The part for which $\delta>\tilde{d}$ and its complement. The supremum over the subdomain for which $\delta>\tilde{d}$ has been calculated above. Next, we use the estimates for the behavior of the solution near $\Gamma_{\text {shock }}$ to obtain the supremum over the complement. By the interpolation inequality, let $\gamma_{1}=\frac{\gamma_{V}}{2}$, we can obtain

$$
d^{2-\gamma_{1}}\|\rho\|_{2} \leq K_{V} \quad \text { for all } d<d_{V},
$$

where $K_{V}$ is independent of $K$. Therefore, we can choose $\tilde{d} \leq \frac{\min \left\{d_{0}, d_{V}\right\}}{2}$ in (17.3) small enough that $\tilde{d}^{1-\gamma_{1}+\mu} C \leq K$. Therefore, (7.2) is satisfied, and we have chosen the parameters $K, K_{0}$, and $\alpha_{0}$ defining $\mathcal{W}$ so that $T$ maps $\mathcal{W}$ into itself.

Now, by the Schauder fixed point theorem, there exists a fixed point $\rho$ such that $T \rho=\rho \in C_{\left(-\gamma_{1}\right)}^{2}$. Then $\rho$ is a solution of the boundary value problem (3.2) and (3.5) -(3.7) and meets the estimates listed in the lemma.

2. We now show the three properties listed in this lemma for the fixed boundary nonlinear problem (3.2) and (3.5) - (3.7). First we prove property (i):

$$
c^{2}\left(\rho^{\varepsilon, \delta}\right)-r^{2} \geq 0 \quad \text { in } \bar{\Omega}^{\varepsilon, \delta},
$$

by the maximum principle.

On contrary, we assume that there exists a nonempty set $D=\{(\xi, \eta) \in \bar{\Omega}$ : $\left.c^{2}(\rho)-r^{2}<0\right\}$. Then it is easy to check that $P_{2} \notin D$. Since $O \notin D$,

$$
D \subset \Omega_{s}:=\left\{X \in \bar{\Omega} \backslash V: r^{2}>\bar{c}^{2}\left(\rho, \rho_{0}\right)\right\},
$$

where $V$ is the set of the corner points of $\Omega$. 
Firstly, inside $\Omega_{s}$, multiplying $(\gamma-1) \rho^{\gamma-2}$ both sides of the equation $Q^{\varepsilon,+} \rho=0$, and denoting $c^{2}(\rho)=\rho^{\gamma-1}=u$, we have

$$
\begin{aligned}
L u & =(\gamma-1) \rho^{\gamma-2} Q^{\varepsilon,+} \rho \\
& =\sum_{i=1}^{2} a_{i i}^{\varepsilon}\left(D_{i i} u-\frac{\gamma-2}{\gamma-1} \frac{1}{\rho^{\gamma-1}}\left|D_{i} u\right|^{2}\right)+\zeta^{\prime}\left(c^{2}-r^{2}\right)\left(c^{2}-r^{2}\right)_{r} u_{r}+\frac{1}{r^{2}} u_{\theta}^{2}+b^{\varepsilon} u_{r} \\
& =0 .
\end{aligned}
$$

We note that $\frac{\varepsilon}{2} \leq a_{11}^{\varepsilon} \leq \varepsilon$ due to the cut-off function $\zeta$ in $D$. We evaluate $L r^{2}$ in $D$ :

$$
\begin{aligned}
L r^{2} & \geq-2 \varepsilon\left|1-\frac{2(\gamma-2)}{\gamma-1} \frac{1}{\rho^{\gamma-1}} r^{2}\right|+\zeta^{\prime}\left(c^{2}-r^{2}\right)\left(c^{2}-r^{2}\right)_{r} u_{r}+2 c^{2} \\
& \geq 2 r_{0}^{2}-\frac{2 \varepsilon}{\rho_{0}^{\gamma-1}}\left|\rho_{0}^{\gamma}-1-\frac{2|\gamma-2|}{\gamma-1} r_{0}^{2}\right|>0
\end{aligned}
$$

with small $\varepsilon<\varepsilon_{0}:=\frac{\rho_{0}^{\gamma-1} r_{0}^{2}}{\left|\rho_{0}^{\gamma-1}-\frac{2|\gamma-2|}{\gamma-1} r_{0}^{2}\right|}$ when $\left(c^{2}-r^{2}\right)_{r}=0$. Then it means that the minimum point of $c^{2}-r^{2}$ can not obtained in $D$.

Secondly, along $\Gamma_{\text {shock }} \cap D$, Multiplying $(\gamma-1) \rho^{\gamma-2}$ over the equation $M \rho=0$, we have the boundary condition for $u$ :

$$
0=(\gamma-1) \rho^{\gamma-2} M \rho=\tilde{M} u=\sum_{i=1}^{2} \beta_{i} D_{i} u .
$$

At the same time, we have

$$
\tilde{M} r^{2}=2 r \beta_{1}=2 r r^{\prime}\left(c^{2}\left(r^{2}-\bar{c}^{2}\right)-3 \bar{c}^{2}\left(c^{2}-r^{2}\right)\right)>0 \quad \text { on } \Gamma_{\text {shock }} \cap D,
$$

where we have used the fact that $r^{2} \geq c^{2} \geq \bar{c}^{2}$ in $\Omega_{s}$. Thus it means that the minimum point of $c^{2}-r^{2}$ can not obtained along $\Gamma_{\text {shock }} \cap D$.

Thirdly, on $\Gamma_{0} \cap D$,

$$
(\gamma-1) \rho^{\gamma-2} \frac{\partial \rho}{\partial \boldsymbol{\nu}}-\frac{\partial r^{2}}{\partial \boldsymbol{\nu}}=0
$$

which is a contradiction due to the Hopf maximum principle. Therefore, there is no minimum point, which implies that the set $D=\emptyset$. This completes the proof of property (i). We remark here that property (i) guarantees the ellipticity of our nonlinear system, so that we can remove the cut-off function.

3. We can show property (ii), i.e.,

$$
r-\bar{c}\left(\rho, \rho_{0}\right) \geq 0 \quad \text { on } \Gamma_{\text {shock}} .
$$

The proof is similar to 12 based on Lemma 3.6. The main idea is to assume that there exists a non-empty set $B=\left\{X \in \overline{\Gamma_{\text {shock }}}: \bar{c}\left(\rho, \rho_{0}\right)-r>0\right\}$ and a point $X \in B$ such that

$$
\max _{\bar{B}}\left(\bar{c}^{2}\left(\rho, \rho_{0}\right)-r^{2}\right)=\left(\bar{c}^{2}-r^{2}\right)(X)=m>0 .
$$

It is clear that $X \neq P_{1}, P_{2}$. Therefore, if $X$ exists, then $X \in \Gamma_{\text {shock }} \backslash\left\{P_{1}, P_{2}\right\}$. Then $X$ can be either a local maximum point or a saddle point in $\Omega \cup \Gamma_{\text {shock }}$. We show that both cases can not occur, which implies that such $X$ does not exist. The case that $X$ is a local maximum point is proved by the maximum principle. For the 
more complicated case that $X$ is a saddle point, then multiplying $\left(\bar{c}^{2}\right)^{\prime}$ both sides of $Q^{\varepsilon} \rho=0$ yields

$$
L \bar{c}^{2}=\sum_{i=1}^{2} a_{i i}^{\varepsilon} D_{i i}\left(\bar{c}^{2}\right)+a_{1}\left(\bar{c}^{2}\right)_{r}^{2}+a_{2}\left(\bar{c}^{2}\right)_{\theta}^{2}+\tilde{b}^{\varepsilon}\left(\bar{c}^{2}\right)_{r},
$$

where $a_{1}=-a_{11}^{\varepsilon}+\frac{a}{\left(\bar{c}^{2}\right)^{\prime}}$ and $a_{2}=-a_{22}^{\varepsilon}+\frac{a}{r^{2}\left(\bar{c}^{2}\right)^{\prime}}$.

Since $X$ is a saddle point, we can construct a barrier function $\psi$ so that $X=$ $\left(r_{x}, \theta_{x}\right)$ is a maximum point along the normal direction.

We define $d:=r_{x}-r+r^{\prime}\left(\theta_{x}\right)\left(\theta-\theta_{x}\right)$ and a set

$$
W:=\{(r, \theta) \in \Omega: d>0\} \cap\left\{(r, \theta) \in \Omega: \bar{c}^{2}-r^{2}>m\right\} .
$$

Set $u=\bar{c}^{2}-r^{2}-m$, and let

$$
w:=\frac{1}{\mu_{0}}\left(e^{\mu_{0} u}-1\right), \quad \mu_{0}>0 .
$$

Choose $\mu_{0}=\frac{\max \left\{a_{i}\right\}}{e_{0}}$, where $a_{11}^{\varepsilon}, a_{22}^{\varepsilon} \geq e_{0}>0$ in $W$, and $\mu_{0}$ and $e_{0}$ are independent of $\varepsilon$. Thus, we find $\psi(d)$ to be

$$
\psi=\frac{m_{0} b_{1}+d_{1} f_{1}}{b_{1}} \frac{1-e^{-b_{1} d / e_{0}}}{1-e^{-b_{1} d_{1} / e_{0}}}-\frac{f_{1}}{b_{1}} d,
$$

which satisfies the boundary condition:

$$
\psi(0)=0, \quad \psi\left(d_{1}\right)=m_{0},
$$

with $m_{0}=\frac{e^{\mu_{0} u_{\max }-1}}{\mu_{0}}$, where $u_{\max }=\max _{W} u=\max _{W}\left(\bar{c}^{2}-r^{2}-m\right)$ and $d_{1}>0$. Here $b_{1}=\max _{\bar{W}}\left(4 a_{1} r+b\right), f_{1}=\max _{W} e^{\mu_{0} u}\left(2 a_{11}^{\varepsilon_{0}}+4 r^{2} a_{1}+2 r \tilde{b}^{\varepsilon}\right)^{+}$, and $\varepsilon \leq \varepsilon_{0}$.

Hence, in the set $W$, using the maximum principle and (2.5), at $X$, we finally have

$$
\begin{aligned}
0 & \geq 2 r \beta_{1}-\psi^{\prime} \mu d_{r}(X)+\beta_{2}\left(\psi^{\prime} d \theta+\beta_{2} \psi^{\prime} r^{\prime} d r\right)(X) \\
& =2 r \beta_{1}+\psi^{\prime}(0)\left(\beta_{1}-r^{\prime} \beta_{2}\right) \\
& =2 r \beta_{1}+\psi^{\prime}(0) \mu .
\end{aligned}
$$

On the other hand, by the Taylor series expansion, we can show that, for sufficiently small $d_{1}$ and $\vartheta=O\left(d_{1}\right)$, we have

$$
2 r \beta_{1}+\psi^{\prime}(0) \mu>0
$$

which is a contradiction. Therefore, there is no such $X$, which implies that the set $B=\emptyset$.

4. We study the monotonicity of $\rho$ along the shock boundary $\Gamma_{\text {shock}}$, which will be used to describe the behavior of $\rho^{\varepsilon, \delta}$ and $r^{\varepsilon, \delta}$ near the shock $\Gamma_{\text {shock }}^{\varepsilon, \delta}$ when $\varepsilon, \delta$ tend to zero, and the convexity of the shock in the $(\xi, \eta)$-coordinates.

The proof is technical, which can be followed as in [4], with the main difference that we only need the uniform $C^{\alpha}$-regularity. We only list the major procedure and 
the difference. For simplicity, we write $\rho=\rho^{\varepsilon, \delta}$ below. To prove the monotonicity, we argue by contradiction.

First, we examine the $C^{\alpha}$-function $\rho$ restricted to $\Gamma_{\text {shock }}$. Without confusion, we may order the points along $\Gamma_{\text {shock }}$ by $\theta$ and refer to the intervals along $\Gamma_{\text {shock }}$ by the label. Then the lack of monotonicity implies that there exist points $\Theta_{1}$ and $\Theta_{2}$ on $\Gamma_{\text {shock}}$, with $P_{2}<\Theta_{1}<\Theta_{2}<P_{1}$, at which $\rho\left(\Theta_{1}\right)>\rho\left(\Theta_{2}\right)$. Thus we immediately deduce that

(a). In $\left(P_{2}, \Theta_{2}\right)$, there exists $\tilde{C}$ with $\rho(\tilde{C})=\max _{\left[P_{2}, \Theta_{2}\right]} \rho$;

(b). In $\left(\tilde{C}, P_{1}\right)$, there exists $D$ with $\rho(D)=\min _{\left[\tilde{C}, P_{1}\right]} \rho$.

We want to identify points $C$ and $D$ on $\Gamma_{\text {shock }}$ with $C<D$ such that

(i) $\rho\left(P_{2}\right) \leq \rho \leq \rho(C)$ on $\left[P_{2}, C\right]$;

(ii) $\rho(C) \geq \rho \geq \rho(D)$ on $[C, D]$;

(iii) $\rho(D) \leq \rho \leq \rho\left(P_{1}\right)$ on $\left[D, P_{1}\right]$.

Now, property (ii) may not hold with $C=\tilde{C}$ because $\rho(\tilde{C})$ is the maximum value of $\rho$ only at the interval $\left[P_{2}, D\right]$, and we may have $D>\Theta_{2}$. Then, if there is a point in $\left(P_{2}, \Theta_{2}\right)$ at which $\rho>\rho(\tilde{C})$, we let $C$ to be the point. Otherwise, we choose $C=\tilde{C}$. Thus, all the three properties hold.

Now we look at the function $\rho$ in $\Omega$. The idea is to partition $\Omega$ into three subdomains by two curves $\Gamma_{C}$ and $\Gamma_{D}$ from $C$ and $D$ to points $A$ and $B$ respectively on $\Gamma_{0}$, in such a way $\rho(A)>\rho(B)$ that we can deduce that there is a point $m$ on $\Gamma_{0}$ at which $\rho$ obtains a maximum on either the subdomain $\Omega_{A}$ or the domain $\Omega_{B}$, thus violating the Hopf maximum principle. This is also the case even if it happens to be the origin $O$. It suffices to show that $\rho(m)$ is the maximum value of $\rho$ on the boundary of $\Omega_{A}$ or $\Omega_{B}$.

We now construct the Lipschitz curves on which $\rho$ has certain monotone property. That is,

$$
\begin{array}{lll}
\rho(A) \geq \rho \geq \rho(C)-\mu & \text { on } \Gamma_{C}, & \rho(A)>\rho(C), \\
\rho(B) \leq \rho \leq \rho(D)+\mu & \text { on } \Gamma_{D}, & \rho(B)<\rho(D),
\end{array}
$$

for certain number $\mu>0$. We specify

$$
\mu=\frac{1}{4} \min \left\{\rho(C)-\rho(D), \rho_{1}-\rho(C), \rho(D)-\bar{\rho}\right\} .
$$

Since $\rho \in C^{\alpha}(\bar{\Omega})$, we have

$$
\left|\rho\left(X_{1}\right)-\rho\left(X_{2}\right)\right| \leq M\left|X_{1}-X_{2}\right|^{\alpha}
$$

for some $M>0$ and $X_{1}, X_{2} \in \bar{\Omega}$. Now, on any ball with radius $r>0$,

$$
\operatorname{Osc}(\rho) \leq 2 M r^{\alpha} \text {. }
$$

Let $R=\left(\frac{\mu}{2 M}\right)^{-\alpha}$. We have

$$
\operatorname{Osc}_{B_{R} \cap \Omega}(\rho) \leq \mu
$$


Now $\Gamma_{C}$ can be constructed as follows (cf. Fig. (5): In $B_{R}(C) \cap \Omega$, let $X_{1}$ be a point at which $\rho$ attains its maximum value in $\overline{B_{R}(C)}$. Then the first segment of $\Gamma_{C}$ is a straight line from $C$ to $X_{1}$ and, on the segment, we have

$$
\rho(X) \geq \rho(C)-\mu, \quad \rho(X) \leq \rho\left(X_{1}\right) .
$$

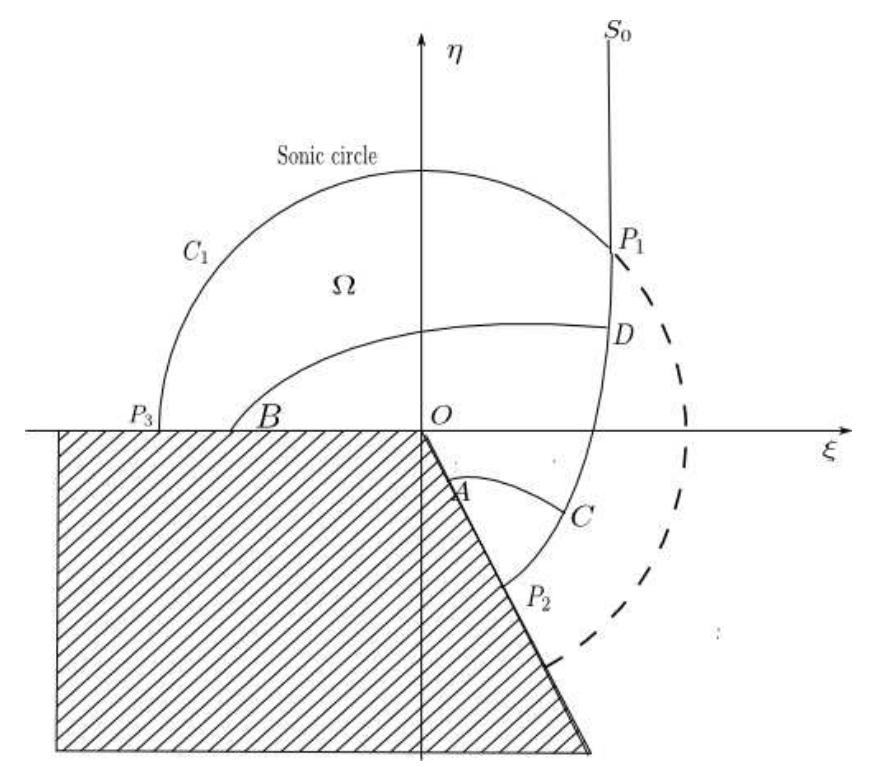

Figure 5. Hypothetical Curves

Now we continue inductively, forming a sequence of the line segments with corners at $\left\{X_{i}\right\}$ (take $X_{0}=C$ ), along which $\rho(X) \geq \rho(C)-\mu$ and $\rho\left(X_{1}\right)<\rho\left(X_{2}\right)<\cdots$. Since the domain $\Omega$ is finite, this process must end at finite steps when we reach a point $X_{L}=B \in \partial \Omega$. Similarly, we construct $\Gamma_{D}$, with termination point $A \in \partial \Omega$.

We now locate $A$ and $B$. We note that the two curves cannot cross each other. Furthermore, $\Gamma_{C}$ cannot terminate at $\Gamma_{\text {sonic }}$ where $\rho>\rho_{1}-\mu>\rho(D)+\mu$. For the same reason, it can not come back to $\Gamma_{\text {shock }}$ in $\left[P_{2}, C\right]$ or $[C, D]$ where $\rho \leq \rho(C)$. Finally, $A$ cannot lie in the segment $\left[P_{2}, C\right]$ of $\Gamma_{\text {shock }}$. Hence, $A$ has to end on $\Gamma_{0}$. Similarly, $B$ cannot lie on $\Gamma_{\text {shock }}$ where $\rho \geq \rho(D)$ in the interval $\left[D, P_{1}\right)$ and must lie on $\Gamma_{0}$ (see Fig. 5).

Now we reach to our final contradiction. Since $\rho(A)$ is larger than $\bar{\rho}$ and $\rho(B)$, there is a point $m$ along the boundary $P_{2} O B$ at which $\rho$ attains a maximum. Assume first that $m$ is not the origin, then $m$ can not be a local maximum for the domain $\Omega$ by the Hopf lemma. However, along the entire boundary of the domain $\mathrm{P}_{2} C D B P_{2}$, $\rho \leq \rho(m)$, which implies that it is a maximum. This is a contradiction. Now, if $m$ coincides with $O$, the similar minimum point $X$ resembling $B$ can not coincide with $O$. We can find that there is no place for such $X$ either. Thus, this is also a contradiction. We conclude that $\rho$ is monotone along $\Gamma_{\text {shock }}$ from $P_{2}$ to $P_{1}$. 
Acknowledgement. The authors would like to thank Mikhail Feldman for helpful discussions and suggestions. The research of Gui-Qiang Chen was supported in part by the National Science Foundation under Grants DMS-0935967 and DMS-0807551, the UK EPSRC Science and Innovation Award to the Oxford Centre for Nonlinear PDE (EP/E035027/1), the NSFC under a joint project Grant 10728101, and the Royal Society-Wolfson Research Merit Award (UK). Xuemei Deng's research was supported in part by China Scholarship Council No. 2008631071 and by the EPSRC Science and Innovation Award to the Oxford Centre for Nonlinear PDE (EP/E035027/1). The research of Wei Xiang was supported in part by China Scholarship Council No. 2009610055 and by the EPSRC Science and Innovation award to the Oxford Centre for Nonlinear PDE (EP/E035027/1).

\section{REFERENCES}

[1] Bae, M., Chen, G.-Q., And Feldman, M.: Regularity of solutions to regular shock reflection for potential flow. Invent. Math. 175, 505-543 (2009)

[2] Bargman, V.: On nearly glancing reflection of shocks. Office Sci. Res. and Develop. Rep. No. 5117 (1945)

[3] C̆Anić, S., Keyfitz, B. L., And Kim, E. H.: A free boundary problem for a quasilnear degenerate elliptic equation: Transonic regular reflection of weak shocks. Comm. Pure Appl. Math. 55, 71-92 (2002)

[4] C̆Anić, S., Keyfitz, B. L., And Kim, E. H.: Free foundary problems for nonlinear wave systems: Mach stems for interacting shocks. SIAM J. Math. Anal. 37, 1947-1977 (2006)

[5] Chen, G.-Q. And Feldman, M.: Potential theory for shock reflection by large-angle wedges. Proceedings of the National Academy of Sciences USA (PNAS), 102, 15368-15372 (2005)

[6] Chen, G.-Q. And Feldman, M.: Global solutions to shock reflection by large-angle wedges for potential flow. Ann. Math. 171, 1067-1182 (2010)

[7] Chen, G.-Q. And Feldman, M.: Mathematics of Shock Reflection-Diffraction and von Neumann Conjectures, Monograph, Oxford, 2013

[8] Courant, R. And Friedrichs, K. O.: Supersonic Flow and Shock Waves, Reprinting of the 1948 original. Applied Mathematical Sciences, Vol. 21. Springer-Verlag: New York-Heidelberg, 1976

[9] Fletcher, C. H., Taub, A. H., and Bleakney, W.: The Mach reflection of shock waves at nearly glancing incidence. Rev. Modern Phys. 23(3), 271-286 (1951)

[10] Fletcher, C. H., Weimer, D. K., and Bleakney, W.: Pressure behind a shcok wave diffracted through a small angle. Phys. Rev. 78(5), 634-635 (1950)

[11] Gilbarg, D. and Trudinger, N.: Elliptic Partial Differential Equations of Second Order, 2nd Ed., Springer: Berlin, 1983

[12] KIm, E. H.: A global sub-sonic solution to an interacting transonic shock of the self-similar nonlinear wave equation. J. Diff. Eqs. 248, 2906-2930 (2010)

[13] Lieberman, G.: The Perron process applied to oblique derivative problems. Adv. Math. 55, 161-172 (1985)

[14] Lieberman, G.: Regularized distance and its applications. Pacific J. Math. 117, 329-352 (1985)

[15] Lieberman, G.: Mixed boundary value problems for elliptiv and parabolic differential equation of second order. J. Math. Anal. Appl. 113, 422-440 (1986)

[16] Lieberman, G.: Oblique derivative problems in Lipschitz domains, II. Discontinuous boundary data. J. Reine Angew. Math. 389, 1-21 (1988)

[17] Lieberman, G.: Optimal Hölder regularity for mixed boundary value problems. J. Math. Anal. Appl. 143 , 572-586 (1989) 
[18] Lighthill, M. J.: The diffraction of blast I. Proc. Roy. Soc. 198A, 454-470 (1949)

[19] Lighthill, M. J.: The diffraction of blast II. Proc. Roy. Soc. 200A , 554-565 (1950)

[20] SERre, D.: Multidimensional shock interaction for a Chaplygin gas. Arch. Ration. Mech. Anal. $191,539-577(2009)$

[21] Whitham, G. B.: Linear and Nonlinear Waves. Reprint of the 1974 original. A WileyInterscience Publication. John Wiley \& Sons, Inc.: New York, 1999

[22] Zheng, Y.: Two-dimensional regular shock reflection for the pressure gradient system of conservation laws. Acta Math. Appl. Sinica. (English Ser) 22 , 177-210 (2006).

Gui-Qiang G. Chen, Mathematical Institute, University of Oxford, Oxford, OX2 6GG, UK; School of Mathematical Sciences, Fudan University, Shanghai 200433, China; Department of Mathematics, Northwestern University, Evanston, IL 60208, USA

E-mail address: chengq@maths.ox.ac.uk

Xuemei Deng, Mathematical Institute, University of Oxford, Oxford, OX2 6GG, UK; School of Mathematical Sciences, Xiamen University, Xiamen, Fujian 361005, CHInA

E-mail address: dmeimeisx@yahoo.com.cn

Wei Xiang, School of Mathematical Sciences, Fudan University, Shanghai 200433, China; and Mathematical Institute, University of Oxford, Oxford, OX2 6GG, UK

E-mail address: 071018004@fudan.edu.cn; xiang@maths.ox.ac.uk 